\title{
Partial Volume Correction of Cardiac Mouse Positron Emission Tomography Images
}

\author{
by \\ Tyler Dumouchel, M.Sc.
A thesis submitted to the
Faculty of Graduate and Postdoctoral Affairs
in partial fulfillment of the requirements
for the degree of
Doctor of Philosophy \\ Ottawa-Carleton Institute for Physics \\ Department of Physics, Carleton University \\ Ottawa, Ontario, Canada \\ October, 2011 \\ (C) Copyright 2011, Tyler Dumouchel
}


Library and Archives

Canada

Published Heritage

Branch

395 Wellington Street

Ottawa ON K1A ON4

Canada
Bibliothèque et

Archives Canada

Direction du

Patrimoine de l'édition

395 , rue Wellington

Ottawa ON K1A ON4

Canada
Your file Votre référence

ISBN: 978-0-494-87746-3

Our file Notre référence

ISBN: $978-0-494-87746-3$

\section{NOTICE:}

The author has granted a nonexclusive license allowing Library and Archives Canada to reproduce, publish, archive, preserve, conserve, communicate to the public by telecommunication or on the Internet, loan, distrbute and sell theses worldwide, for commercial or noncommercial purposes, in microform, paper, electronic and/or any other formats.

The author retains copyright ownership and moral rights in this thesis. Neither the thesis nor substantial extracts from it may be printed or otherwise reproduced without the author's permission.
AVIS:

L'auteur a accordé une licence non exclusive permettant à la Bibliothèque et Archives Canada de reproduire, publier, archiver, sauvegarder, conserver, transmettre au public par télécommunication ou par l'Internet, prêter, distribuer et vendre des thèses partout dans le monde, à des fins commerciales ou autres, sur support microforme, papier, électronique et/ou autres formats.

L'auteur conserve la propriété du droit d'auteur et des droits moraux qui protege cette thèse. $\mathrm{Ni}$ la thèse ni des extraits substantiels de celle-ci ne doivent être imprimés ou autrement reproduits sans son autorisation.
In compliance with the Canadian Privacy Act some supporting forms may have been removed from this thesis.

While these forms may be included in the document page count, their removal does not represent any loss of content from the thesis.
Conformément à la loi canadienne sur la protection de la vie privée, quelques formulaires secondaires ont été enlevés de cette thèse.

Bien que ces formulaires aient inclus dans la pagination, il n'y aura aucun contenu manquant. 


\section{Abstract}

The left ventricle $(\mathrm{LV})$ myocardial wall thickness of mice is on the order of small animal positron emission tomography (PET) scanner spatial resolution. As a result partial volume (PV) losses diminish the quantitative accuracy of cardiac $\left[{ }^{18} \mathrm{~F}\right]$-FDG mouse PET images by up to $50 \%$. In this work, novel PV correction strategies are explored for improving quantitative accuracy of electrocardiogram-gated LV myocardial images without the need of anatomical imaging.

A 1D/2D method was developed, based on a radial 1D spatial model of the PET activity distribution across the LV myocardium. A 2D LV image was created using myocardial wall borders and blood/background activities derived from the 1D model. A 2D PET scanner resolution model was applied to the 2D image to obtain estimates of the true myocardial activity. The 1D/2D method restored LV myocardial activity in 2D simulated LV images to within 5\% while improving image uniformity. However, PET is a $3 \mathrm{D}$ imaging modality thus the $1 \mathrm{D} / 2 \mathrm{D}$ model was not sufficient for describing the $3 \mathrm{D}$ nature of the LV.

To overcome this limitation, a 1D/3D method was developed that was implemented in the same manner as the $1 \mathrm{D} / 2 \mathrm{D}$ method using a 3D resolution model. This algorithm restored quantitative accuracy in simulated images to within $10 \%$ while 
improving image uniformity. The method underestimated myocardial activity in the apex region of the heart due to the $1 \mathrm{D}$ model's inability to fully describe the 3D curvature in this region.

Finally, a fully 3D technique was developed that eliminated the dependence on the ID model. In this case LV and resolution models were described in 3D. The method restored myocardial activity to within $2 \%$ for simulated and measured phantoms while improving image uniformity. Performing the fully 3D method with in vivo mouse data improved quantitative accuracy to $10 \%$ and improved image uniformity indicating that this technique is a viable PV-correction solution.

Partial volume correction is required to restore quantitative accuracy in cardiac mouse PET imaging. While mouse imaging was considered in this study, the algorithms may be equally applicable to other species, provided a similar relationship between myocardial wall thickness and spatial resolution exists. 


\section{Statement of Originality}

Some of the work contained in this thesis has been published in journals or as abstracts that were presented at conferences. Preliminary results from Chapter 2 were originally presented at Society of Nuclear Medicine Conferences (Dumouchel and deKemp 2008, 2011a). The contents of Chapter 2 were published in the IEEE Transaction on Nuclear Science (Dumouchel and deKemp 2011b). Initial results for the work of Chapter 3 were published in abstract form and presented at the Canadian Organization of Medical Physicists Annual Meeting (Dumouchel and deKemp 2010a). The contents of Chapter 3 were based on work published in the 2010 IEEE Nuclear Science Symposium and Medical Imaging Conference Proceedings (Dumouchel and deKemp 2010b). Finally, some the methods presented in Chapter 4 were initially published in abstract form for the 2011 Society of Nuclear Medicine Conference (Dumouchel and deKemp 2011c).

For each reference, there were two authors including Robert A. deKemp (my supervisor) and myself. In each case I performed the experiments, analyzed the data and created the written/presentation components of the work under my supervisor's guidance. 


\section{Acknowledgement}

Firstly, I would first like to thank my supervisor, Robert deKemp. His guidance and support throughout my tenure at the University of Ottawa Heart Institute have enabled me to excel in my research. He has helped me get over many hurdles and has passed on a lot of knowledge to me.

I am grateful for all of the positive support and friendships that I have developed with my colleagues within the cardiac PET imaging team: Jennifer Renaud, Ran Klein, Myra Kordos, Marc Lamoureux, Chad Hunter and Matthew Efseaff. They have really made my experience here enjoyable.

I would like to thank the PET radiochemistry laboratory for the radiotracers that they have provided me with for my various research projects. I am indebted to Stephanie Thom for all of her help with the mouse experiments in this research.

This research would not have been feasible without the various sources of funding that I was fortunate to achieve. I have greatly appreciated the NSERC, OGS, Physics Departmental, Carleton Domestic Tuition, Kiwanis Club of Ottawa Medical Foundation

and Dr. Kanta Marwah, Wylda Blanche McDermid Holbein Memorial, and John Lyndhurst Kingston Memorial scholarships that I have received. This work was 
supported by grants from the CIHR, Heart and Stroke Foundation of Ontario, Ontario Research Fund and Canadian Foundation for Innovation.

Finally, this work would have not been possible without the encouragement from my friends and family who supported me every step of the way. I would like to especially thank my lovely wife Ashley Dumouchel. She has played a significant role in my education by providing me with advice, daily support and confidence. I am truly grateful to share my life with such a wonderful person. 


\section{Table of Contents}

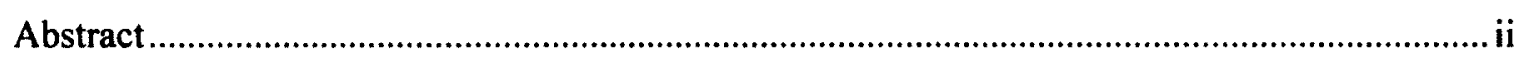

Statement of Originality ................................................................................................... iv

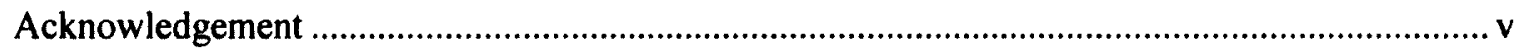

Table of Contents .......................................................................................................

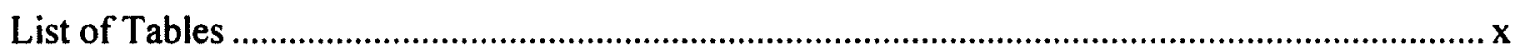

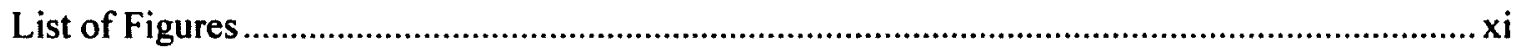

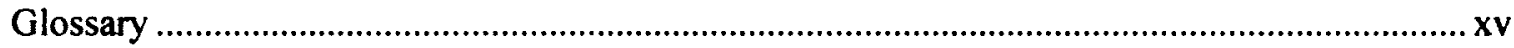

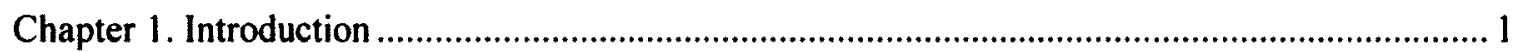

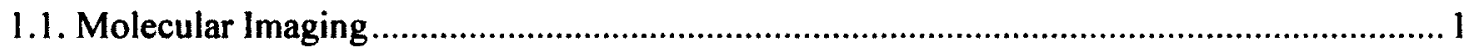

1.2. Small-Animal Positron Emission Tomography .................................................................... 3

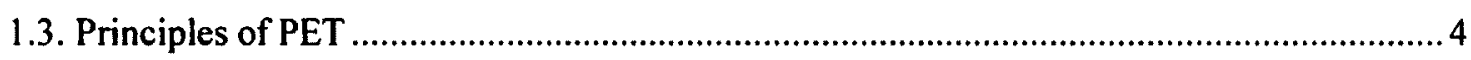

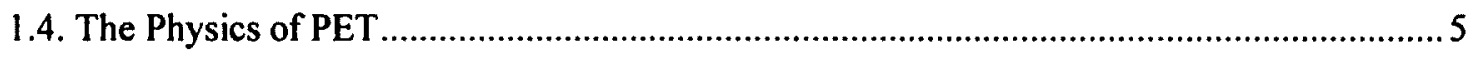

1.5. Coincidence Detection ................................................................................................

1.6. The Inveon Small-Animal PET Scanner........................................................................... 10

1.7. Data Corrections in PET ................................................................................... 11

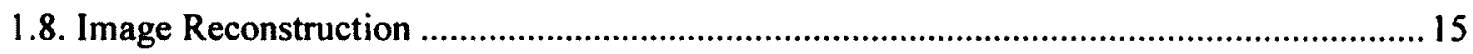

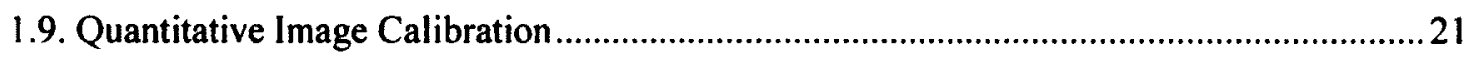

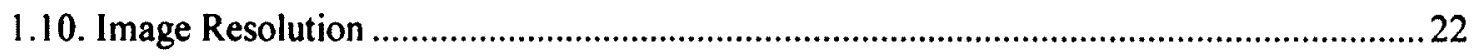

1.11. The Partial Volume Effect in Cardiac PET Imaging …………….................................23

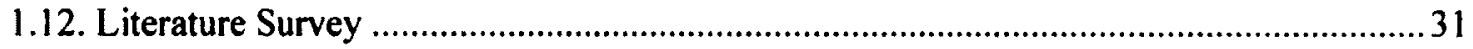

Chapter 2. A Partial Volume Correction Strategy for Cardiac Mouse PET Imaging using a

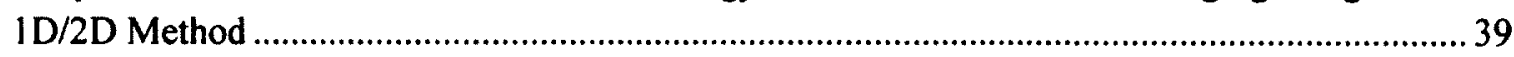

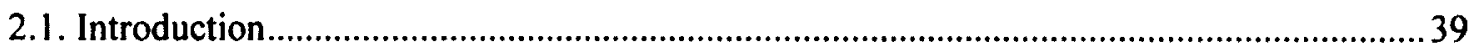




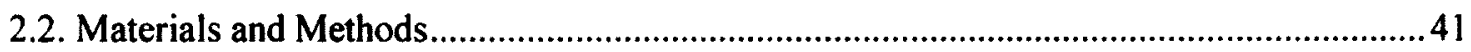

2.2.1. The 1D/2D Partial Volume Correction Algorithm...................................................41

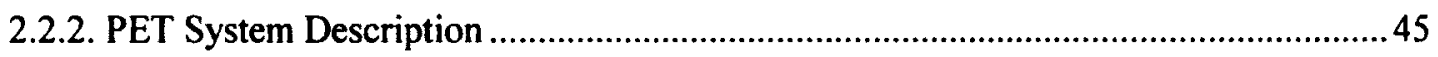

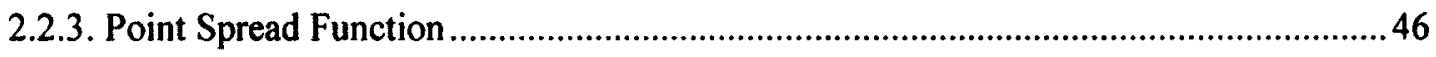

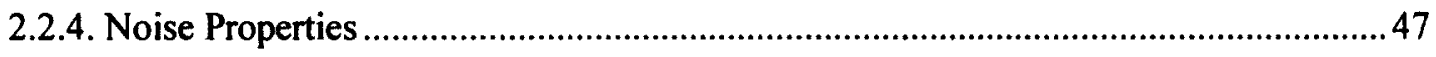

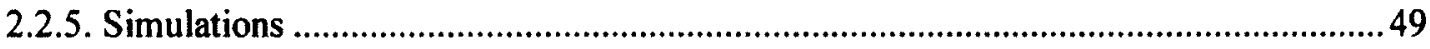

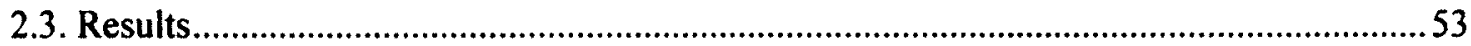

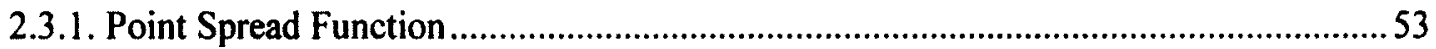

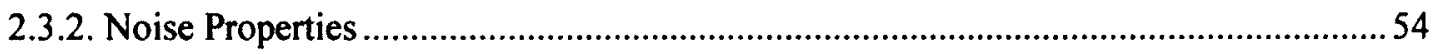

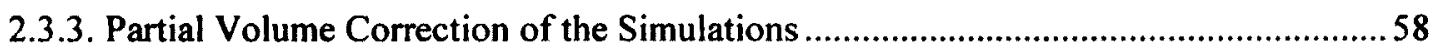

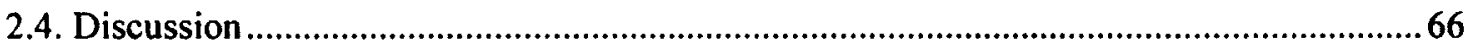

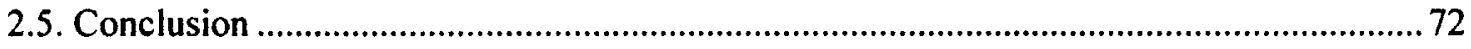

Chapter 3. Experimental Validation of the 1D/2D Partial Volume Correction Method and

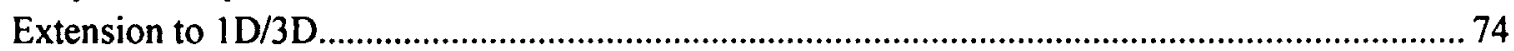

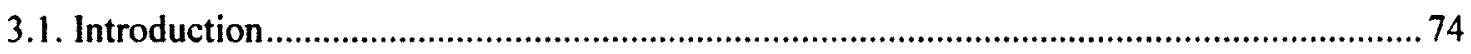

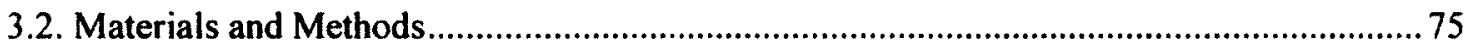

3.2.1. The 1D/2D Partial Volume Correction Algorithm.................................................... 75

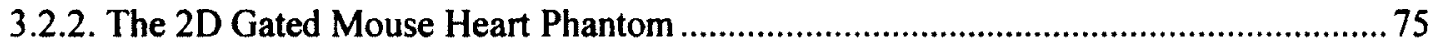

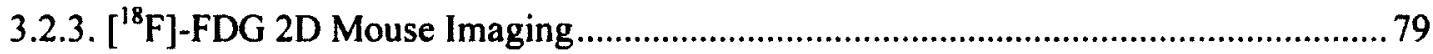

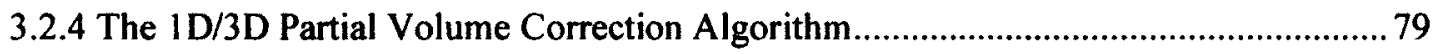

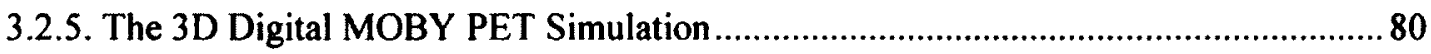

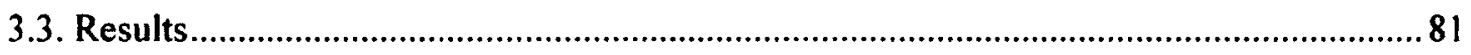

3.3.1. 1D/2D PV Correction of the 2D Gated Mouse Heart Phantom ...................................81

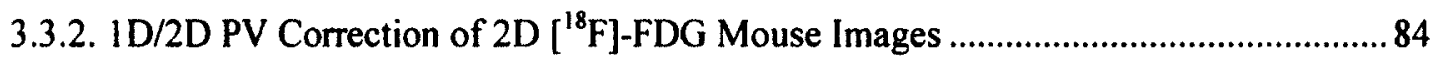

3.3.3. 1D/3D PV Correction of the 3D Digital MOBY PET Simulation ...............................85

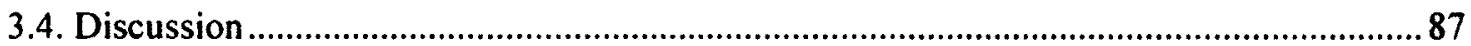

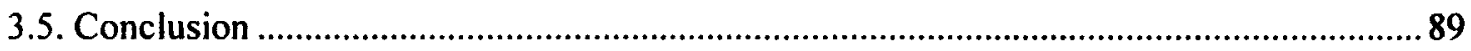

Chapter 4. A Three-Dimensional Model-Based Partial Volume Correction Strategy for Gated Cardiac Mouse PET Imaging: Principle and Validation ..................................................91

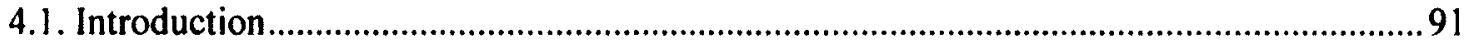

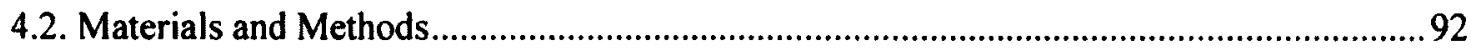

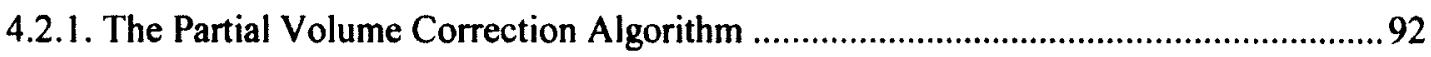

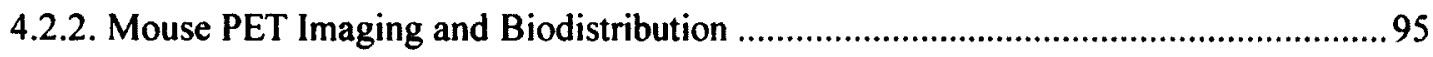

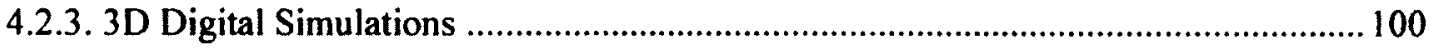

viii 


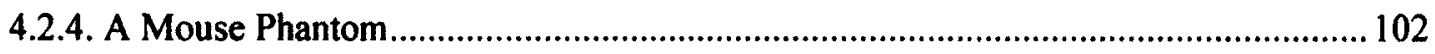

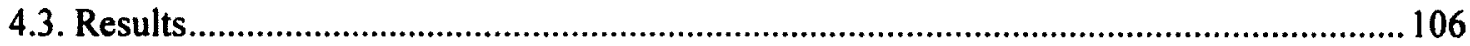

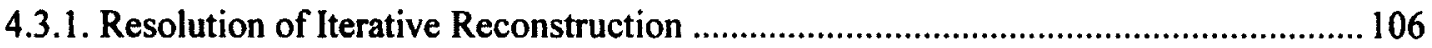

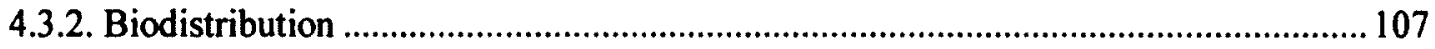

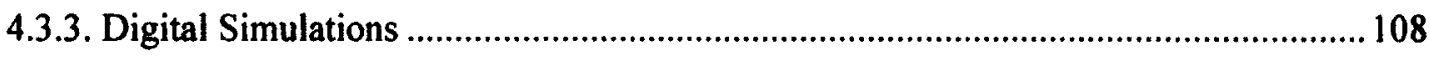

4.3.4. Mouse Phantom Simulations and Measurements .................................................. 113

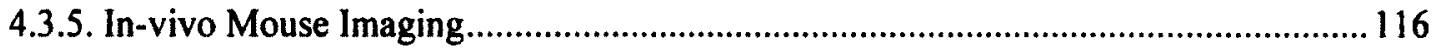

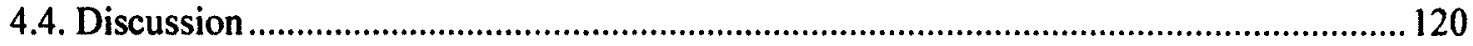

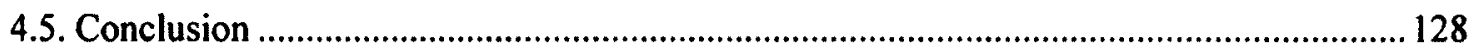

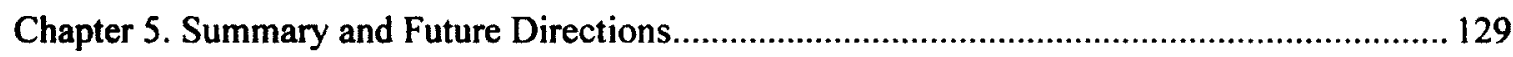

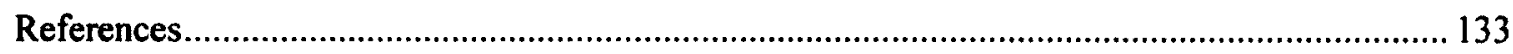




\section{List of Tables}

Table 1.1. Commonly used PET isotopes (Bacharach 1992) ................................................4

Table 1.2. Interaction probabilities for $511 \mathrm{keV}$ photons in various materials (Cherry and

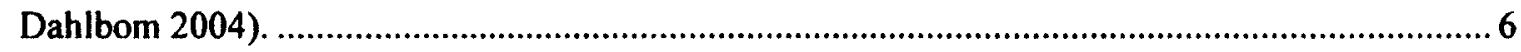

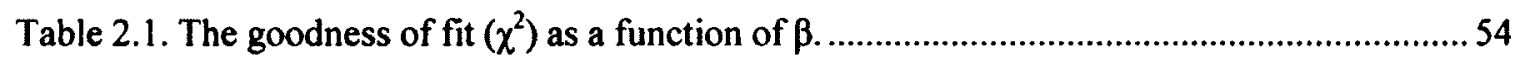

Table 2.2. Evaluation of SNR in the liver for two time frames. .............................................. 57

Table 2.3. Relative mean uptake values \pm SD from mice injected with $\left[{ }^{18} \mathrm{~F}\right]-\mathrm{FDG}$. ..................58

Table 2.4. Errors on Wall Thickness Estimates from the 1D Model. ...........................................61

Table 3.1. 1D/2D PV correction results for the 2D simulated and measured data. ..................... 82

Table 3.2. 1D/2D PV correction results for the $\left[{ }^{18} \mathrm{~F}\right]$-FDG cardiac mouse PET images. ..............84

Table 3.3. 1D/3D PV correction results for the 3D simulated phantom......................................85

Table 4.1. Mean (SD) transverse and axial FWHM resolution measurements........................... 107

Table 4.2. Mean (SD) $\left[{ }^{18} \mathrm{~F}\right]$-FDG uptake in the liver 55 minutes post-injection. ........................ 108

Table 4.3. Mean (SD) biodistribution values taken over the 10 mice at 60 minutes post-

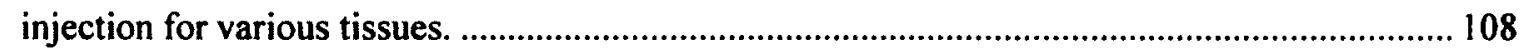

Table 4.4. PV-corrected 3D MOBY simulations with and without noise. ...............................110

Table 4.5. PV-corrected 3D mouse phantom results for simulated and measured data with

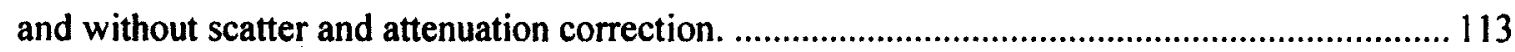

Table 4.6. PV-corrected mouse LV myocardium values without corrections for attenuation and scatter.

Table 4.7. PV-corrected mouse LV myocardium values with corrections for attenuation and scatter. 


\section{List of Figures}

Figure 1.1. The different types of coincidences in PET illustrated with a cross section through a torso. The dashed lines represent the photon trajectories while the solid lines represent the coincidence detection line. In the case of the true coincidence, the initial photon trajectories follow the same path as the coincidence lines.

Figure 1.2. Sinogram sampling variables illustrated on a transverse view $\left(\phi=0^{\circ}\right)$ and on an axial view $\left(\theta=90^{\circ}\right)$ through the scanner's field of view. Oblique sinograms are obtained by sampling as a function of $\mathrm{r}, \theta$ and $\phi$. 2D sinograms can be sampled by setting $\phi=0$.

Figure 1.3. The measurement of a 2D activity distribution into sinogram space. The intensity in each sinogram pixel is obtained by computing the line integral through the activity distribution at a particular $r$ and $\theta$. The color bar on the right gives the relative intensity for the activity distribution and the sinogram in arbitrary units. The large arrow pointing to a row on the sinogram corresponds to the angle of the LORs drawn on the activity distribution.

Figure 1.4. Relative activity distribution through a transverse slice of the MOBY phantom with the LV and RV myocardium having the highest relative activity

Figure 1.5. The effect of resolution degradation is illustrated with an FDG-like organ distribution on the cross section of the MOBY phantom. The top row shows the true activity distribution. The middle row displays the simulated measured PET images and the bottom row displays profiles across the activity distributions, with the location of each profile indicated on each image by the dashed line. The first, second and last columns show the relative activity distributions for the LV myocardium, blood/background and total body respectively. The dashed profiles represent $A_{\text {rrue }}$ while the darker profile represents $A_{\text {PETT }}$.

Figure 1.6. RC versus wall thickness for a FWHM Gaussian resolution of $1.4 \mathrm{~mm}$.

Figure 1.7. The 3D view of $A_{\text {True }}$ and $A_{P E T}$ of the $\mathrm{LV}$ at end-diastole from the MOBY phantom. The locations of the transverse, coronal and sagittal views are located on $A_{\text {True }}$ and are denoted by dashed lines on the corresponding images. In this case, the $\mathrm{LV}$ long axis is oriented along the $z$ dimension, while the short axis is in $(x, y)$. The base and apex of the heart are indicated on the sagittal view of $A_{P L T}$. Sampling directions for polar map 
formation are indicated on $A_{\text {PkT. }}$. A color map is shown on the right to give the relative activity distributions of the images.

Figure 1.8. Polar maps for a 3D activity distribution in the LV myocardium. The left image shows a uniform polar map for an LV myocardial activity distribution that does not suffer from PV losses, while the right image demonstrates a measured PET image that suffers from non-uniform PV effects. Polar map sampling is indicated on the ideal activity distribution. A color map is shown on the right, which gives the relative activity distribution of both polar maps. Polar maps are generated using FlowQuant (Klein et al. 2010).

Figure 2.1. Illustration of the $1 \mathrm{D}$ convolution used to approximate the PV effects in the myocardium. $C_{m}$ is the true myocardial activity, $C_{b}$ is the blood activity, $C_{g}$ is the background activity, $R$ defines the location of the myocardial/blood border, $w$ is the width of the LV myocardium, $\sigma$ defines the width of the Gaussian (FWHM $=2.355 \sigma$ ), erf is the error function, $\otimes$ is the $1 D$ convolution operator and $x$ is the radial position along the profile. $\theta$ and $g$ denote that the values are obtained as a function of radial angle and gate respectively.

Figure 2.2. Illustration of the proposed PV correction process on a simulated MOBY phantom: (A) A gated short axis end-diastolic PET image of the LV myocardium was selected and profiles were taken across the image; one such profile is shown on the image. (B) Radial profiles (circles) were fit using the equation in Figure 2.1. The 1D fitted information, namely $w(\theta), R(\theta), C_{g}(\theta)$ and $C_{b}(\theta)$ were used to construct $(\mathrm{C})$ an estimated $2 \mathrm{D}$ background and blood image and (D) an estimated 2D heart image with uniform activity equal to unity. $(E)$ and $(F)$ represent the convolution of images $(C)$ and $(D)$ respectively with the PSF, thus (F) is an image of the regional RCs. (G) represents a transverse slice of the PET image (A) with blood and background removed. An estimate of the true activity in the myocardium can be obtained with the ratio $(H)$ sampled in the region of the myocardium along a profile angle $\theta$. This process was repeated for all gates (g).

Figure 2.3. Illustration of Phantom 3 in (a) end-diastole and (b) end-systole. The wall thicknesses ranged from $0.2-1.6 \mathrm{~mm}$ and $0.2-1.8 \mathrm{~mm}$ in end-diastole and end-systole respectively. The ejection fraction was $32 \%$. Phantom 2 has a similar appearance but with concentric contraction.

Figure 2.4. Resolution (FWHM) as a function of $\beta$. The results were obtained by fitting a Gaussian function to profiles obtained from images of a line source.

Figure 2.5. Image of a transverse plane of the uniform syringe. (a) The measured PET image and (b) a simulated image with SNR $=12.5$.

Figure 2.6. Squared SNR as a function of NEC and the true coincidences for a uniform activity distribution with a diameter comparable to a mouse. The linear fits are illustrated by the dashed lines.

Figure 2.7. Recovery of activity as a function of the average wall thickness. Each point represents one angle through the myocardium. The dashed line represents the true activity. The dots represent the maximum recovery in the images without partial volume correction. 
The open circles give the myocardium estimation with the $1 \mathrm{D}$ model $\left(C_{m, 1 D}(\theta)\right)$, while the stars represent the results with the 1D/2D model $\left(C_{m, 2 D}(\theta)\right)$. (a) The results for phantom 2 and (b) the results for phantom 3 . Note that the results from phantom 3 have a larger range in the average wall thickness simulated.

Figure 2.8. Difference between the estimated model $I_{m y s}$ and the true phantom myocardium in end-diastole and end-systole for phantoms 1-3.

Figure 2.9. End-diastolic images of the simulated MOBY phantom at five minutes postinjection for different SNRs.

Figure 2.10. The effect of noise on the partial volume recovery algorithm in the MOBY phantoms. (a) Background values are derived from the early time frame of Table 2.3 and (b) background values are derived from the late time frame of Table 2.3. The recovery prior to correction is denoted with the asterisks while the corrected values are denoted by the open circles.

Figure 2.11. Recovery versus relative background activities in (a) phantom 1, (b) phantom 2 and (c) phantom 3. The bottom graphs have an SNR of infinity while the top graphs have an SNR of 15.2

Figure 3.1. The 2D variable wall thickness mouse heart phantom used to test the 1D/2D PV correction algorithm.

Figure 3.2. Illustration of the 3D sampling process for the base and apex regions on a simulated ECG gated PET image of the heart. The spherical profile sampling $(r, \theta, \phi)$ of the apex is shown on the coronal image. The cylindrical $(r, \theta, z)$ sampling performed in the base region is illustrated on the transverse image. The location of each respective image is indicated by the dashed line on the corresponding image.

Figure 3.3. Cross section of the mouse heart phantom between the end-systolic and enddiastolic phases: (a) Raw digital phantom image. (b) Simulated image with Gaussian FWHM $=1.3 \mathrm{~mm}$ and (c) the measured image with $1800 \mathrm{~s}$ of experimental data (OSEM3D/MAP, $\beta=1$ ).

Figure 3.4. Average corrected activity versus average wall thickness for the (a) simulated and (b) measured data with $1800 \mathrm{~s}$ of data.

Figure 3.5. Average activity (top) and NU (bottom) versus plane for the 3D simulated MOBY heart phantom. Planes are ordered from apex to base, with the base region starting at plane 11. The NU was calculated as the standard deviation divided by the mean activity value. The ID/2D PV correction algorithm was only performed on slices $11-42$. .86

Figure 4.1. Schematic illustration of the fully 3D PV correction algorithm. .94

Figure 4.2. Illustration of the location of the point sources in the base and apex regions on an end-diastolic PET image of a $28 \mathrm{~g}$ mouse injected with $32 \mathrm{MBq}$ of $\left[{ }^{18} \mathrm{~F}\right]$-FDG. (a) Four point source measurements were obtained along the cardinal directions on one mid-LV plane. (b) One point source measurement was taken at the apex. The point source locations are indicated by the white dots. The dashed black line on image (a) shows the location of 
the cross section for image (b). The gray arrows indicate the point source location on each respective image.

Figure 4.3. The end-diastolic LV mouse phantom with fillable LV and liver chambers. Notable dimensions are shown on the figure. View A illustrates a side view through the phantom that intersects through middle of the LV and liver chambers. View B is a cross section of the LV region to demonstrate the LV dimensions. The dashed lines of view $\mathrm{C}$ indicate that only a portion of the phantom was selected. The purpose of view $C$ is to show the injection ports for the $\mathrm{LV}$

Figure 4.4. Example radial profile across a measured PSF (open circles) with the fitted profile (dashed line). The data shown were taken from mouse 4 at end-diastole with the point source placed at the basal lateral wall location.

Figure 4.5. Plot of the squared SNR measured in the mice livers as a function of relative activity concentration. The open circles represent the measured data while the straight line is the linear fit $\left(R^{2}>0.99\right)$.

Figure 4.6. Polar maps of the myocardial RCs for the 3D simulated MOBY images with and without noise. The polar map for noise realization 5 from Table 4.4 is displayed.

Figure 4.7. Plot of recovery coefficient versus plane, organized from apex to base. The 3D simulated MOBY data (a) without noise and (b) with noise. The error bars of corrected recovery in (b) are computed as the standard deviation across the different noise realizations. Error bars for uncorrected activity fall within the points.

Figure 4.8. The 3D mouse phantom. The transverse and coronal images of the simulated phantom are shown on the left while the transverse and coronal images of the measured phantom with correction for attenuation and scatter are shown on the right.

Figure 4.9. Polar maps for the 3D mouse phantom. The top/bottom rows are the polar maps without/with PV correction. The first column is the polar maps for the simulated phantom while the second and third columns are the polar maps for the measured phantom without and with corrections for scatter and attenuation respectively.

Figure 4.10. Illustration of the PV correction for mouse 4. The LV images at end-diastole with attenuation and scatter correction are shown on the top row. Polar maps, averaged across all gates, before (middle row) and after (bottom row) PV correction are presented for images without (left) and with (right) scatter and attenuation correction. Images are on a common scale for comparison.

Figure 4.11. Percent bias as a function of change in initial conditions for MOBY simulation. (a) Change in initial wall thickness and (b) change in initial activity. 122 


\section{Glossary}

$\begin{array}{ll}\text { ID } & \text { One-dimension } \\ \text { 2D } & \text { Two-dimension } \\ \text { 3D } & \text { Three-dimension } \\ \text { ACF } & \text { Attenuation correction factor } \\ \text { CT } & \text { Computed tomography } \\ \text { ECG } & \text { Electrocardiogram } \\ \text { FBP } & \text { Filtered-backprojection } \\ \text { FDG } & \text { Fluorodeoxyglucose } \\ \text { FVB } & \text { Friend Leukemia Virus B } \\ \text { FWHM } & \text { Full width at half maximum } \\ \text { GTM } & \text { Geometric transfer matrix } \\ \text { LOR } & \text { Line of response } \\ \text { LSO } & \text { Lutetium oxyorthosilicate } \\ \text { LV } & \text { Left ventricle } \\ \text { MAP } & \text { Maximum } a \text { posteriori } \\ \text { MLEM } & \text { Maximum-likelihood expectation maximization } \\ \text { MOBY } & \text { Mouse body } \\ \text { MRI } & \text { Magnetic resonance imaging } \\ \text { MRS } & \text { Magnetic resonance spectroscopy } \\ \text { NEC } & \text { Noise equivalent count } \\ \text { NEMA } & \text { National Electrical Manufacturers Association } \\ \text { NMF } & \text { Normalization multiplication factor } \\ \text { NU } & \text { Non-uniformity } \\ \text { OSEM } & \text { Ordered-subset expectation maximization } \\ \text { PET } & \text { Positron emission tomography } \\ \text { PMT } & \text { Photomultiplier tube } \\ \text { PSF } & \text { Point spread function } \\ \text { PV } & \text { Partial volume } \\ \text { RC } & \text { Recovery coefficient } \\ \text { ROI } & \text { Region of interest } \\ \text { RV } & \text { Right ventricle } \\ & \end{array}$


$\mathrm{SD}$ SNR

SPECT

VOI
Standard deviation

Signal-to-noise-ratio

Single photon emission computed tomography

Volume of interest 


\section{Chapter 1. Introduction}

\subsection{Molecular Imaging}

Molecular imaging is defined as the non-invasive visualization and measurement of biological function at the molecular and cellular levels in living systems (Sinusas et al. 2008, Pysz et al. 2010). Available imaging modalities include optical imaging, ultrasound, magnetic resonance imaging (MRI), magnetic resonance spectroscopy (MRS), X-ray computed tomography (CT), single photon emission computed tomography (SPECT) and positron emission tomography (PET). Optical imaging permits fast acquisitions and is highly sensitive $\left(10^{-17}-10^{-6} \mathrm{~mol} / \mathrm{L}\right)$ and quantitative, but lacks the ability to perform whole-body imaging, due to the limited depth penetration of the photons (Sinusas et al. 2008). Ultrasound imaging provides high spatial and temporal resolution, and is also sensitive $\left(10^{-9}-10^{-6} \mathrm{~mol} / \mathrm{L}\right)$. However, whole-body imaging is not permitted, and contrast agents are only available for vascular space imaging (Pysz et al. 2010). MRI has high spatial resolution and provides excellent soft tissue contrast. This allows for anatomic image generation, including whole-body imaging, in conjunction with physiological imaging. However, MRI has limited sensitivity $\left(10^{-5}-10^{-3} \mathrm{~mol} / \mathrm{L}\right)$ for 
the detection of contrast agents (Sinusas et al. 2008, Pysz et al. 2010). MRS can also be performed, but still suffers from low sensitivity $\left(10^{-5}-10^{-3} \mathrm{~mol} / \mathrm{L}\right)$. X-ray CT is another modality that enables high resolution whole-body anatomic imaging, however, the application of this modality for examining molecular probes is currently in the early developmental stages (deKemp et al. 2010). The advantage with CT is that it can be combined with the nuclear imaging modalities to add anatomical context.

PET and SPECT utilize tracers which are molecules that follow specific physiological processes, and have an attached radioisotope. Isotopes are selected that decay by positron or gamma-ray emission for PET and SPECT respectively. These nuclear imaging modalities have inferior spatial resolution compared to MRI, CT and ultrasound. However, both modalities permit whole-body imaging and have high sensitivity $\left(10^{-12}-10^{-10} \mathrm{~mol} / \mathrm{L}\right)$, and along with MRS, are used for the majority of clinical molecular imaging (Sinusas et al. 2008, Pysz et al. 2010). PET technology tends to be more expensive than SPECT technology, largely due to the fact that an onsite cyclotron is required to generate many of the radioisotopes. SPECT imaging allows imaging of multiple tracers simultaneously and SPECT radioisotopes typically have longer half-lives in comparison to the isotopes used in PET. PET has the advantage that radioisotopes produced can be attached to the tracers such that the isotope does not alter the chemical nature of the tracer. For example ${ }^{11} \mathrm{C}$, a positron emission isotope, can theoretically be attached to any physiological molecule containing carbon (e.g. $\left[{ }^{11} \mathrm{C}\right]$-glucose). PET has greater detection sensitivity $(>10 \times)$ in comparison to SPECT, enabling dynamic imaging and a greater ability for absolute image quantification (Sinusas et al. 2008). Although PET resolution is inferior to SPECT resolution in the case of small-animal preclinical 
systems, the resolution of clinical PET is higher than SPECT. PET has found a variety of clinical applications in oncology, neurology and cardiology and has recently found applications for small animal studies.

\subsection{Small-Animal Positron Emission Tomography}

With growing interest in studying animal models of human disease, small-animal PET has become an invaluable research tool. The technology is also facilitating proliferation of clinical PET by providing a conduit to examine animal models of human disease, develop new radiopharmaceuticals and accelerate new therapies within a controlled environment. Small-animal PET also enables new PET technology to be tested prior to being introduced into a clinical setting. Before the introduction of small-animal PET imaging, multiple animals were required to study preclinical tracer kinetics, because animals needed to be sacrificed at different time points. Noninvasive in vivo imaging permits longitudinal studies, wherein one animal can be studied at different time points with the advantage that each animal serves as its own control. This eliminates errors due to physiologic variability between animals.

There are several prototype and commercially available small-animal PET scanners (Hutchins et al. 2008, Levin and Zaidi 2007). The small-animal PET scanner used in this work is the Inveon dedicated PET (Siemens Preclinical Solutions, Knoxville, TN, USA) which is the successor of the preclinical PET system developed by Cherry et al. (1997). 


\subsection{Principles of PET}

Nuclei with a high proton to neutron ratio may decay by either positron emission or electron capture. Positron decay converts an atomic proton into a neutron releasing a positron and an electron neutrino following equation 1.1:

$$
{ }_{2}^{A} X \rightarrow{ }_{Z-1}^{A} Y+\beta^{+}+v_{e}
$$

where $X$ is the parent isotope, $Y$ is the daughter isotope, $A$ is the atomic number, $Z$ is the number of protons, $\beta^{+}$is a positron and $v_{e}$ is an electron neutrino.

Some of the commonly used PET isotopes are displayed in Table 1.1, along with their mean/maximum energies and mean/extrapolated ranges in water (Bacharach 1992). The first four isotopes in the table are produced with a cyclotron, while ${ }^{82} \mathrm{Rb}$ is produced in a generator from the decay of ${ }^{82} \mathrm{Sr}$. The $3.35 \mathrm{MeV}$ positron from ${ }^{82} \mathrm{Rb}$ decay occurs $83 \%$ of the time. Twelve percent of the time, a $2.57 \mathrm{MeV}$ positron is released in the decay (Bacharach 1992).

TABLE 1.1. COMMONLY USED PET ISOTOPES (BACHARACH 1992).

\begin{tabular}{cccccc}
\hline Isotope & $\begin{array}{c}\text { Half Life } \\
(\mathrm{min})\end{array}$ & $\begin{array}{c}\text { Max Energy } \\
(\mathrm{MeV})\end{array}$ & $\begin{array}{c}\text { Mean } \\
\text { Energy } \\
(\mathrm{MeV})\end{array}$ & $\begin{array}{c}\text { Extrapolated } \\
\text { Range }(\mathrm{mm})\end{array}$ & $\begin{array}{c}\text { Mean Range } \\
(\mathrm{mm})\end{array}$ \\
\hline${ }^{18} \mathrm{~F}$ & 109.8 & 0.64 & 0.25 & 2.3 & 0.35 \\
${ }^{11} \mathrm{C}$ & 20.3 & 0.96 & 0.39 & 4.1 & 0.56 \\
${ }^{13} \mathrm{~N}$ & 10.0 & 1.19 & 0.49 & 5.2 & 0.72 \\
${ }^{15} \mathrm{O}$ & 2.1 & 1.72 & 0.74 & 8.1 & 1.10 \\
${ }^{82} \mathrm{Rb}$ & 1.3 & 3.35 & 1.52 & 16.7 & 2.40 \\
\hline
\end{tabular}

Positrons lose their kinetic energy via inelastic collisions within millimeters of a decay event in water and annihilate with an electron producing two photons that are emitted at $180^{\circ}$ from each other, each carrying an energy of $511 \mathrm{keV}$. It is possible for 
more than two photons to be emitted, but this occurs in less than $0.003 \%$ of the annihilations (Cherry and Dahlbom 2004). The electron and positron may be in motion when annihilation occurs, and to conserve momentum, the photon trajectories can deviate from being $180^{\circ}$ apart from each other. This phenomenon is known as noncollinearity, and the distribution of angles follows a Gaussian distribution with a mean of $180^{\circ}$ and a full width at half maximum (FWHM) approximately equal to $0.5^{\circ}$ (Cherry and Dahlbom 2004).

To perform PET imaging, radioactive tracers are injected into the subject. PET tracers are created by attaching a positron emitting isotope to a molecule that follows a natural biological process. Tracers either directly follow, or are analogue to the biological process. A tracer that directly follows the process is formed when a native atom in the molecule is directly substituted with the radioisotope (e.g. ${ }^{11} \mathrm{C}$ substitutes the ${ }^{12} \mathrm{C}$ in $\mathrm{CO}_{2}$ to yield $\left.\left[{ }^{11} \mathrm{C}\right]-\mathrm{CO}_{2}\right)$. In contrast, an analogue is formed when radioisotope substitution changes the chemical nature of the biological molecule. For example, replacing the $\mathrm{OH}^{-}$ group with an ${ }^{18} \mathrm{~F}^{-}$in a glucose molecule results in a radioactive glucose analogue called ${ }^{18}$ F-fluorodeoxyglucose $\left(\left[{ }^{18} \mathrm{~F}\right]-\mathrm{FDG}\right)$.

\subsection{The Physics of PET}

Each photon from positron annihilation passes through the subject prior to being detected. The $511 \mathrm{keV}$ photons may undergo coherent scattering, photoelectric absorption or Compton scattering within body tissue. At these energies, pair production is not energetically feasible and the coherent scattering cross section is negligible. Thus, the 
dominant gamma-ray interactions are photoelectric absorption and Compton scattering. Photoelectric absorption occurs when all of the gamma-ray energy is transferred to an orbital electron in the medium, stripping an atomic electron. In Compton scattering the gamma-ray scatters off a free or loosely bound electron in the medium transferring part of the photon energy to the electron. The scattered photon loses energy in the interaction and changes direction. The energy of the scattered photon $\left(E_{s}\right)$ can be written in terms of the initial energy of the photon $\left(E_{i}\right)$ and the scattering angle $(\varphi)$ :

$$
E_{s}=\frac{m_{e} c^{2}}{\frac{m_{e} c^{2}}{E_{i}}+1-\cos \varphi}
$$

where $c$ is the speed of light and $m_{e}$ is the rest mass of an electron. The residual energy in the interaction is transferred to the recoil electron.

TABLE 1.2. INTERACTION PROBABILITIES FOR $511 \mathrm{KEV} \mathrm{PHOTONS} \mathrm{IN} \mathrm{VARIOUS} \mathrm{MATERIALS}$ (CHERRY AND DAHLBOM 2004).

\begin{tabular}{cccc}
\hline Material & $\mu_{C}\left(\mathrm{~cm}^{-1}\right)$ & $\mu_{p}\left(\mathrm{~cm}^{-1}\right)$ & $\mu\left(\mathrm{cm}^{-1}\right)$ \\
\hline Soft Tissue/Water & 0.096 & 0.00002 & 0.096 \\
Bone & 0.169 & 0.001 & 0.17 \\
Lead & 0.76 & 0.89 & 1.78 \\
Tungsten & 1.31 & 1.09 & 2.59 \\
Lutetium oxyorthosilicate (LSO) & 0.58 & 0.30 & 0.88 \\
\hline
\end{tabular}

The probability of a photoelectric $\left(\mu_{p}\right)$ or Compton $\left(\mu_{c}\right)$ interaction depends on the composition of the material. For $511 \mathrm{keV}$ photons, the probability of the photoelectric effect occurring per electron scales roughly as $Z^{3,4}$, while the Compton effect scales slowly with $Z$ as it depends on the number of electrons in the medium (Cherry and Dahlbom 2004). The probability of interaction per unit distance $\left(\mathrm{cm}^{-1}\right)$ is given in Table 
1.2 for some materials (Cherry and Dahlbom 2004). The probability that a photon will be attenuated or removed from its original trajectory is given by the linear attenuation coefficient $(\mu)$. At $511 \mathrm{keV}$ the linear attenuation coefficient is described by the photoelectric and Compton interaction probabilities:

$$
\mu=\mu_{p}+\mu_{c}
$$

The interaction by absorption and scatter of a monoenergetic beam of photons in a narrow-beam geometry can be described by:

$$
I=I_{o} e^{-\int \mu(x) d x}
$$

where $I_{o}$ is the initial beam intensity and $I$ is the intensity after passing through a material of thickness $d x$. In the case of PET, a broad-beam geometry is applicable, because photons that undergo Compton scatter can be detected. Thus, equation 1.4 will underestimate the true number of photons in the presence of scatter.

\subsection{Coincidence Detection}

The simultaneous detection or coincidence detection of the annihilation photons that are travelling $180^{\circ}$ apart from each other is the basis for PET imaging. A coincidence event indicates that an annihilation event occurred somewhere along the line joining two detectors, which is referred to as a line of response (LOR). A coincidence timing window, $2 \tau$, is set to minimize the probability that photons from uncorrelated events are detected in coincidence. The term $\tau$ describes the FWHM precision of the coincidence timing pulse (typically $2-5 \mathrm{~ns}$ ). Coincidences that are detected within the timing window are referred to as prompt coincidences. There are three types of prompt coincidences 
detected including true, random and scattered coincidences as illustrated in Figure 1.1. A true coincidence occurs when both annihilation photons reach opposing detectors without interacting in body tissue. A scattered coincidence is recorded when one or both of the annihilation photons undergo a Compton scatter prior to being detected. An energy acceptance window is set to help reject some of the scattered events as scattered photons carry less energy according to equation 1.2. However, as in the case of the timing window, the energy window has to be large enough to consider the energy resolution of the detector so that unscattered photons are not rejected. A random (or accidental) coincidence event occurs when two photons from separate annihilations are detected within the timing window. This occurs when a photon from each annihilation event is lost because of photon attenuation or because the photon has left the detection system. The random event rate can be described by the following relationship:

$$
R=2 \tau S_{1} S_{2}
$$

where $R$ is the random event rate, and $S_{l}$ and $S_{2}$ are the single photon event rates at detectors 1 and 2 respectively. Neglecting detector deadtime losses, the singles rates are directly proportional to the amount of radioactivity in the field of view. Therefore, the randoms rate is proportional to the square of the activity.

Scatter and random coincidence events result in mispositioned coincidences. Left uncorrected, these events degrade PET image quality, so they must be corrected to preserve accuracy. Attenuation of the true event rate occurs when one or both photons undergo a scatter, or are absorbed by the material in the field of view, or do not have enough energy to overcome the energy window. In this case, no coincidence event is 
recorded. This phenomenon degrades image accuracy by decreasing the expected number of recorded events.

To form a PET image, coincidence events are recorded for a large number of radioactive decay events from all of the LORs that intersect the scanner's threedimensional (3D) field of view. These data are then reconstructed as discussed in Section 1.8 .

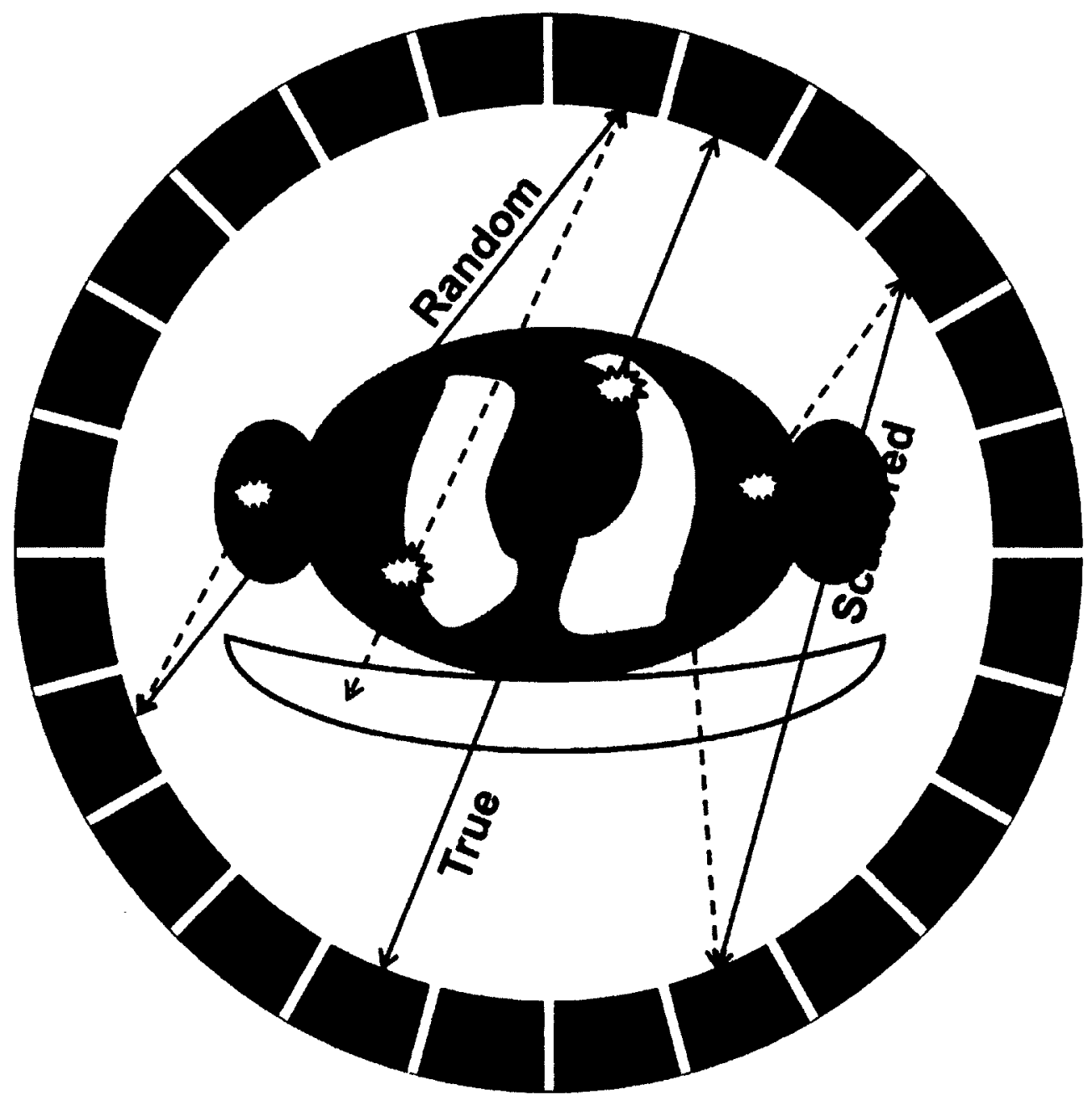

Figure 1.1. The different types of coincidences in PET illustrated with a cross section through a torso. The dashed lines represent the photon trajectories while the solid lines represent the coincidence detection line. In the case of the true coincidence, the initial photon trajectories follow the same path as the coincidence lines. 


\subsection{The Inveon Small-Animal PET Scanner}

The Inveon small-animal dedicated PET scanner (Siemens Preclinical Solutions, Knoxville, TN, USA) is designed for the imaging of rats and mice. The Inveon provides the high count rate and resolution necessary for small-animal imaging (Bao et al. 2009, Constantinescu and Mukherjee 2009, Kemp et al. 2009, Visser et al. 2009). The scanner is constructed from LSO scintillator crystals coupled to photomultiplier tubes (PMTs). The crystals are organized into $20 \times 20$ detector array blocks which are connected to the PMTs via tapered light guides. The crystals have a $1.51 \times 1.51 \mathrm{~mm}$ front face and a 10 $\mathrm{mm}$ length with a pitch of $1.59 \mathrm{~mm}$ in both transverse and axial directions. The average detector energy resolution is $12.3 \%$ (Mintzer and Siegel 2007). There are a total of 64 detector blocks with 16 blocks covering the transverse field of view and 4 arranged axially. The length of the axial field of view is $12.7 \mathrm{~cm}$ while the diameter of the ring is $16.1 \mathrm{~cm}$ and the system packing fraction is $92 \%$. The scanner has a $10 \mathrm{~cm}$ transverse imaging field of view. The detector configuration is illustrated by Mintzer and Siegel (2007) and Bao et al. (2008).

Data are collected and stored in list-mode format which is an event-by-event listing of the coincidences along with information such as time of an event, LOR address, etc. The Inveon permits acquisition of electrocardiogram (ECG) and respiratory gating trigger signals from the animals which are also stored in the list-mode data. List-mode acquisition permits retrospective binning of the data into user-defined dynamic time frames or into ECG and respiratory gates. The typical energy window used is 350-650 $\mathrm{keV}$, while a coincidence timing window of $3.42 \mathrm{~ns}$ is used. 


\subsection{Data Corrections in PET}

There are several factors that affect PET image quality which must be addressed in order to preserve quantitative accuracy. These include detector dead-time, detector nonuniformities, random coincidence events, isotope decay properties, scatter, attenuation, and finite image resolution.

Detector dead-time refers to the phenomenon whereby a second photon striking the detector cannot be processed, due to the time required to integrate the light signal and record the time, energy and position information of a first event. This causes the recorded event rate as a function of activity to become nonlinear. As the activity is increased, the underestimation of events becomes more apparent and with a high enough activity the event rate can begin to decrease. Left uncorrected, dead-time will cause an underestimation of the true activity distribution in the reconstructed image. This phenomenon is corrected on the Inveon using a calibrated global correction function which is related to the singles rates at the detectors.

Detector efficiencies vary due to individual detector responses which can differ due to the physical dimensions of the crystal, geometrical variations of detectors inside of the scanner, and detector electronics. The process of correcting for these nonuniformities is referred to as normalization. This correction is generally performed by measuring the individual LOR efficiencies by placing a uniform source inside the field of view and scanning for long time periods to ensure high count statistics. The response of each LOR is normalized by applying the individual LOR correction factors. The Inveon applies a component-based normalization (Casey et al. 1995) to correct for efficiencies. The normalization is factored into individual detector efficiencies and a geometrical factor 
that considers the position and the exposed face area of the crystals. The normalization multiplication factor (NMF) for an LOR is defined by (Cherry and Dahlbom 2004):

$$
N M F_{i, j}=\frac{1}{\varepsilon_{i} \varepsilon_{j} g_{i, j}}
$$

where $\varepsilon_{i}$ and $\varepsilon_{j}$ are the individual detector efficiencies at detectors $i$ and $j$ respectively and $g_{i j}$ is the geometric factor for a given LOR. The $g_{i j}$ factors are measured at the factory using very high count statistics and are assumed to be constant over time. The individual detector efficiencies are measured by scanning a uniform ${ }^{68} \mathrm{Ge}$ positron emission source until sufficient count statistics are obtained; the manufacturer recommends 12 billion counts. A normalization scan is performed periodically to account for variations in detector efficiency. By using a component-based approach, the count statistics of the coincidence normalization factors are improved. The number of LORs to be corrected is approximately equal to the square of the number of the detectors. The component-based approach uses only individual detector efficiencies, thus for the same ${ }^{68} \mathrm{Ge}$ scan, the count statistics are improved. The advantage in image quality using a component-based normalization over the LOR based method was illustrated for the Inveon by Visser et al. (2009) who showed improvements in image noise.

Random coincidences contain no spatial information about the activity distribution and thus the accuracy of PET images containing random events is decreased because the background activity is increased leading to a loss of contrast. Randoms correction can be performed by correcting individual LORs using equation 1.5. In this case, the singles event rates at the detectors are combined with the coincidence timing window to estimate the number of random events for each LOR. An alternative method for randoms correction is the delayed coincidence window method. This correction 
technique uses a singles event from one detector occurring at time $t$, and compares this event with a singles event at another detector occurring at a time $t+\Delta t . \Delta t$ is chosen to be large enough such that the photons recorded within each window could not have originated from the same coincidence event. These two uncorrelated singles events are combined as a random coincidence count for the specific LOR. These events are measured over the course of the scan over all the LORs then subtracted from the prompt coincidence data prior to image reconstruction. The method currently implemented on the Inveon scanner is the delayed coincidence method.

Isotope decay needs to be considered in PET imaging due to the short half-lives of the radioisotopes used (Table 1.1). The correction for this effect is performed by applying a global decay correction factor based on the length and start time of the imaging frame. PET isotopes can also decay by electron capture, where the probability of electron capture is characteristic of each isotope. Decay branching is corrected by dividing by the characteristic isotope branching fraction.

As described earlier, photon scatter leads to mispositioned events and thus degrades image quality and accuracy, while photon attenuation leads to a reduction of counts. The longest photon paths originate from the center of the subject, thus both effects are most pronounced at the central location. In PET, the standard method for correcting for attenuation is to measure the attenuation distribution using a secondary transmission scan. Transmission scanning is performed using a source that is positioned outside of the object in the field of view and measuring the number of photons that pass through the object $(I)$. If the initial photon intensity $\left(I_{o}\right)$ is known, and is measured with a 
blank scan without the object present, the attenuation correction factors (ACFs) for a given LOR can be obtained using the following relationship:

$$
A C F=\frac{I_{0}}{l}
$$

The ACFs are multiplied by the recorded true event rate to obtain the number of true events in each LOR, in the absence of photon attenuation. Transmission scanning can be obtained using a rotating positron emission source such as ${ }^{68} \mathrm{Ge}$ (deKemp and Nahmias 1994) or a source that is a single gamma-ray emitter such as ${ }^{137} \mathrm{Cs}$ (Yu and Nahmias 1995, Karp et al. 1995). CT images can also be acquired and coregistered to the PET images to obtain the ACFs by scaling the attenuation coefficients measured from the $\mathrm{CT}$ images to $511 \mathrm{keV}$ (Watson et al. 2004). Alternatively, MRI can be used (Hoffmann et al. 2009) to compute the ACFs. Because an MRI image represents the distribution of hydrogen nuclei, obtaining the ACFs at $511 \mathrm{keV}$ requires conversion of the MRI signal. This is achieved by segmenting the MRI image into different tissue types and assigning the appropriate attenuation coefficient to each tissue. The Inveon dedicated PET system performs attenuation correction using a ${ }^{57}$ Co point source, which is a single gamma-ray emitter. Transmission scans are acquired by rotating the source around the subject while translating the bed through the scanner. An energy window of $120-125 \mathrm{keV}$ is used that focuses on the $122 \mathrm{keV}$ gamma-ray emitted by the source. The narrow energy window permits transmission scanning before or after tracer injection.

Scatter correction is performed prior to attenuation correction. This is to ensure that all scattered photons are removed prior to multiplying each LOR by the corresponding measured ACFs. There are several methods available to correct for the scatter phenomenon (Zaidi and Montandon 2007). Currently, the most popular method is 
the single-scatter simulation as proposed by Watson (2000) and this is the algorithm that is implemented on the Inveon. Emission and transmission data are acquired and reconstructed to obtain images of the approximate activity distribution and attenuation coefficients respectively. The number of single-scattered photons in each LOR is calculated by integrating over the scattering volume while considering the physics of Compton scattering. The emission image is used as an estimate to the relative activity distribution, necessary for determining emitter density. Transmission images are used for identifying the attenuation coefficients for the different tissues types inside the subject. The computed single-scattered distribution is subtracted from the emission data prior to performing attenuation correction and image reconstruction.

\subsection{Image Reconstruction}

With PET, the measurements only reveal that an annihilation has occurred somewhere along the LOR. Due to the relationship between photon speed, the size of small animals and currently available coincidence timing windows for small-animal PET scanners, it is impossible to pinpoint the precise location of an annihilation event along an LOR. Therefore, raw projection data must be reconstructed to obtain images of the estimated activity distribution. Prior to performing image reconstruction, list-mode data are typically histogrammed into sinograms. A sinogram is a matrix where each element corresponds to a line integral through the activity distribution along a particular LOR. The term is coined as sinogram because each pixel in image space traces a sine wave in sinogram space. PET data are sampled over the entire 3D field of view, and thus 3D 
sinograms can be produced by including oblique LORs across the axial field of view of the scanner, defined by angle $\phi$ as illustrated in Figure 1.2.
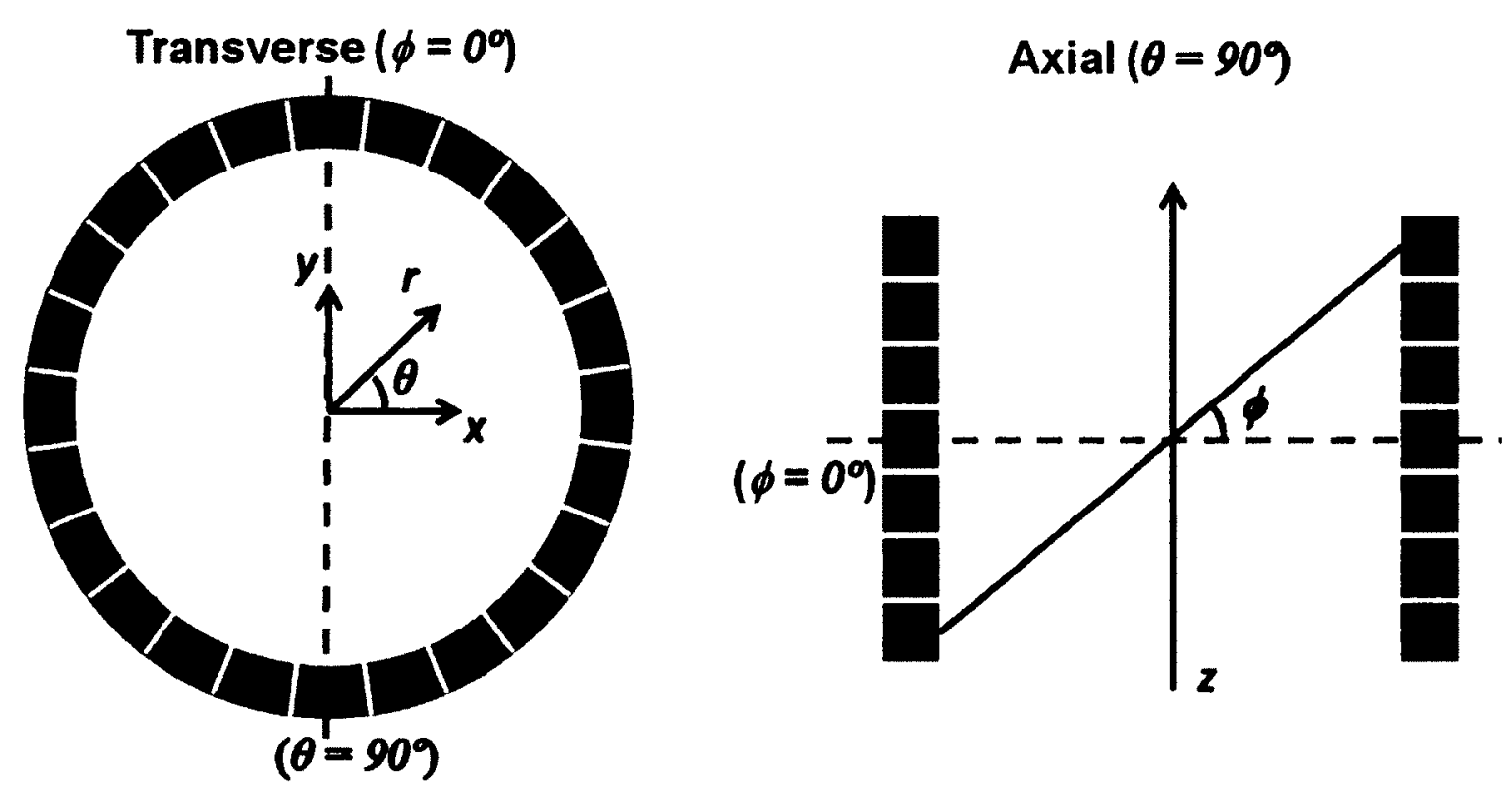

Figure 1.2. Sinogram sampling variables illustrated on a transverse view $\left(\phi=0^{\circ}\right)$ and on an axial view $\left(\theta=90^{\circ}\right)$ through the scanner's field of view. Oblique sinograms are obtained by sampling as a function of $r, \theta$ and $\phi$. 2D sinograms can be sampled by setting $\phi=0$.

Each 3D sinogram pixel location is defined by the radial distance from the center of the field of view $(r)$, the projection angle $(\theta)$ and $\phi$. Figure 1.3 demonstrates how a two-dimensional (2D) transverse cross section through an activity distribution is forward projected into a $2 \mathrm{D}$ sinogram (i.e. $\phi=0$ ). The color bar is used as a guide to give the relative intensity of the activity distribution and the sinogram. The spatial distribution intensity is in units of activity concentration (e.g. $\mathrm{Bq} / \mathrm{mL}$ ) while the sinogram is in units of counts. Thus, the intensity of each sinogram pixel is directly dependent on the length 
of the scan and the activity distribution. A complete set of $3 \mathrm{D}$ sinograms are obtained by sampling the entire field of view by increasing $\phi$. 2D-only sinograms can also be obtained by setting $\phi=0$ and sampling the set of 2D transverse planes as a function of $z$ (Figure 1.2).
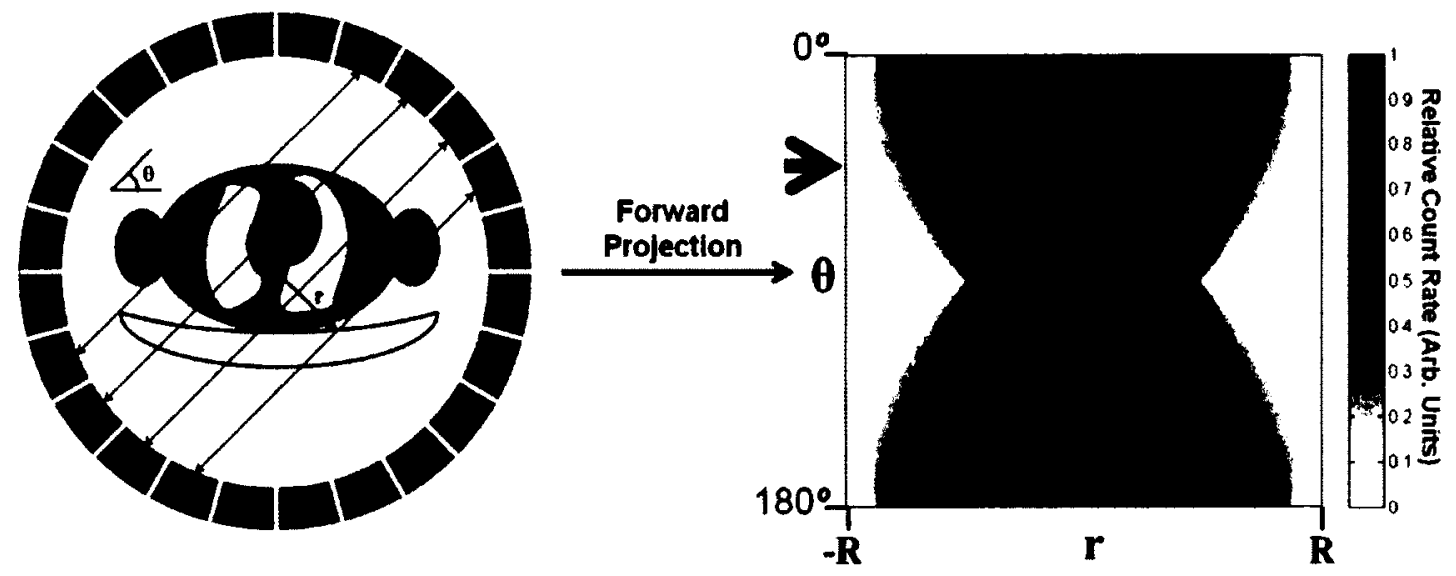

Figure 1.3. The measurement of a 2D activity distribution into sinogram space. The intensity in each sinogram pixel is obtained by computing the line integral through the activity distribution at a particular $r$ and $\theta$. The color bar on the right gives the relative intensity for the activity distribution and the sinogram in arbitrary units. The large arrow pointing to a row on the sinogram corresponds to the angle of the LORs drawn on the activity distribution.

The Inveon considers samples from all LORs transecting the 3D field of view. These $3 \mathrm{D}$ data can be binned to $2 \mathrm{D}$ or $3 \mathrm{D}$ sinograms depending on whether $2 \mathrm{D}$ or $3 \mathrm{D}$ reconstruction is performed, respectively. To perform $2 \mathrm{D}$ reconstruction, the $3 \mathrm{D}$ data are collapsed into a collection of 2D sinograms using Fourier rebinning (Defrise et al. 1997). Each plane is reconstructed separately using the 2D analytic filtered-backprojection 
(FBP) algorithm or the 2D statistical-based ordered-subset expectation maximization (OSEM) algorithm to form a collection of contiguous 2D images which describes the 3D volume. FBP is explained in detail elsewhere (Defrise and Kinahan 1998). For the Inveon, 3D sinograms are obtained with a maximum ring difference of 79 and a span of 3. The ring difference indicates that the LORs are sampled over all possible oblique planes as there are a total of 80 detector planes. The span refers to how many adjacent LORs are included into a particular oblique sinogram at angle $\varphi$ between the transverse and axial directions. Increasing the span will increase the number of counts per bin and will thus improve the noise properties in the reconstructed image. However, as the span increases the axial resolution is degraded because more oblique LORs, which do no intersect along the same lines, are collapsed into a single oblique sinogram (projection mashing). The reconstruction used in this work is 3D OSEM (OSEM3D) in combination with the 3D maximum a posteriori (MAP) algorithm. OSEM and OSEM3D perform similarly but are applied to 2D and 3D sinograms respectively.

The basic principle of OSEM stems from maximum-likelihood expectation maximization (MLEM) which is described as follows. Let $y_{i}$ be the number of recorded coincidences for the $i-t h$ LOR. The mean $\bar{y}_{l}$ is related to the unknown activity distribution $f$ through the following equation (Qi and Leahy 2006):

$$
\bar{y}=P f+r+s
$$

where $r$ is the expected number of random evens, $s$ is the expected number of scattered events, and $P$ is the scanner projection matrix (i.e. the detector response model) which is defined for the Inveon as (Qi et al. 1998a, 1998b):

$$
P=P_{\text {det }, \text { sens }} P_{\text {det }, \text { blur }} P_{\text {attn }} P_{\text {geom }}
$$


where $P_{\text {det,sens }}$ are the normalization factors to correct for detector efficiencies from equation 1.6, $P_{\text {det,blur }}$ models photon noncollinearity, intercrystal scatter and penetration, $P_{a t m}$ are the attenuation correction factors (see Section 1.7), and $P_{\text {geom }}$ is a matrix that gives the probability that a photon pair originating from the activity distribution reaches the front face of particular detector in the absence of attenuation and noncollinearity. The product between $P_{\text {det,sens }}$ and $P_{\text {geom }}$ yields the NMF factor (equation 1.6). Under the assumption that the measured data $y$ follow a Poisson distribution, the maximum likelihood estimate of $f$ can be computed by maximizing the log-likelihood objective function:

$$
L(y \mid f)=\sum_{i=1}^{N} y_{i} \log \left(\bar{y}_{1}\right)-\overline{y_{l}}-\log \left(y_{i} !\right)
$$

where $N$ is the number of LORs. The MLEM reconstruction algorithm is derived by finding the maximum of the log-likelihood equation (Shepp and Vardi 1982, Lange and Carson 1984):

$$
f_{j}^{k+1}=\frac{f_{j}^{k}}{\Sigma_{i} P_{i, j}} \sum_{i} \frac{P_{i, j} y_{i}}{\sum_{j} P_{i, j} f_{j}^{k}+r_{i}+s_{i}}
$$

where $k$ is the iteration number and $j$ is the image voxel. Therefore, the role of the MLEM algorithm is to maximize the log-likelihood function of equation 1.9 by considering the projection data to follow a Poisson distribution. OSEM follows by using only a subset of projection angles within each iteration in order to accelerate convergence (Hudson and Larkin 1994). The reconstruction works by iteratively updating the estimated PET image until there is agreement between measured $(y)$ and estimated projection data $(\bar{y})$.

The objective function $(\Phi)$ for MAP reconstruction differs slightly from equation 1.9. It is formulated from Bayes' theorem. Taking the log of the posterior density of Bayes' theorem, the objective function is (Qi and Leahy 2006): 


$$
\Phi(f)=L(y \mid f)+\ln [p(f)]
$$

where $p(f)$ is the prior probability that is independent of the measured projections and $L(y \mid f)$ is given in equation 1.9. For the Inveon MAP reconstruction, $p(f)$ is modeled as a Gibbs prior with an energy function $U(f)$ and a hyperparameter $\beta$ that controls the local smoothness in a reconstructed image (Qi and Leahy 2000). The objective function is thus:

$$
\Phi(f)=L(y \mid f)-\beta U(f) .
$$

$U(f)$ is chosen to enforce smoothness between neighbouring voxels in a reconstructed image while still enabling abrupt changes between different organs (Qi and Leahy 2006). The actual implementation on the Inveon allows $\beta$ to change as a function of voxel position to encourage uniform resolution across the entire field of view. Equation 1.12 is maximized using the preconditioned conjugate gradient algorithm to obtain an image estimate $f$ with $y$ equal to the 3D sinograms.

The Inveon uses OSEM3D to obtain an initial image estimate and MAP reconstruction to obtain the final image estimate. The fastMAPTM algorithm is used (Cho et al. 2007) which speeds up the MAP algorithm using inverse Fourier rebinning to reduce computational costs from iterating between $3 \mathrm{D}$ sinogram space and the $3 \mathrm{D}$ image volume.

Statistical-based iterative algorithms can be advantageous over analytic methods in terms of signal-to-noise ratio (SNR) and resolution properties. The reconstruction implicitly considers the Poisson nature of the noise in the data, and the solutions give more weight to bins with more counts whereas FBP weights the noise equally throughout the image. Furthermore, statistical-based reconstructions have a system matrix $P$ that models the physics of photon detection which helps to improve image resolution. The 
disadvantage with these reconstructions is that they are nonlinear. A drawback to the OSEM algorithm is that it is not convergent (Qi and Leahy 2006) and overiterating can cause the image noise to increase. Conversely, MAP is convergent and offers a regularized solution to the problem resulting in smooth images with the level of smoothing dependent on the choice $\beta$ from equation 1.12. For these reasons, image reconstruction is typically performed with 2 OSEM $3 \mathrm{D}$ and 18 MAP iterations on the Inveon.

\subsection{Quantitative Image Calibration}

Quantifying PET images in absolute units (e.g. $\mathrm{Bq} / \mathrm{mL}$ ) requires placing a source with known activity concentration inside of the scanner's field of view and determining a calibration factor from the reconstructed image. A source is chosen with a similar geometry compared with the subject of interest to emulate a realistic activity distribution, reducing potential errors due to differences in the physics of photon detection. A long acquisition is performed to minimize errors due to limited count statistics. Data are reconstructed using the same parameters and data corrections (Section 1.7) that are used for the subjects of interest. A region of interest (ROI) is drawn over the activity distribution and the mean pixel intensity within the ROI $\left(A_{R O I}\right)$ is combined with the known activity distribution $\left(A_{T_{r u e}}\right)$ to derive the image calibration factor $\left(\mathrm{Cal}_{M G}\right)$ :

$$
\mathrm{Cal}_{I M G}=\frac{A_{\text {True }}}{A_{\text {ROI }}}
$$

Multiplying $\mathrm{Cal}_{I M G}$ by the reconstructed image intensity produces a calibrated image in units of $A_{\text {True }}$. 
For mouse imaging on the Inveon, a $60 \mathrm{~mL}$ syringe is filled to $30 \mathrm{~mL}$ with 40 $\mathrm{MBq}$ of $\left[{ }^{18} \mathrm{~F}\right]-\mathrm{FDG}$ diluted in water. The cylindrical activity distribution has a similar diameter and length to a mouse. The syringe is scanned for 2 hours and reconstructed with the same reconstruction algorithm parameters and corrections applied that are used for mouse imaging. An ROI is drawn over the central region of the phantom and $\mathrm{Cal}_{\mathrm{MG}}$ is derived.

\subsection{Image Resolution}

The FWHM reconstructed PET image resolution can be described by the following relationship (Moses and Derenzo 1993):

$$
F W H M=a \sqrt{(d / 2)^{2}+b^{2}+s^{2}+(0.0022 D)^{2}}
$$

where $a$ is a factor dependent on the type of reconstruction, $d$ is the detector pitch, $b$ is the position decoding accuracy of the detector, $s$ is the positron range of the isotope and $0.0022 D$ is due to photon noncollinearity with $D$ representing the ring diameter. The factor $a$ can range from 1.0-1.3 depending on the reconstruction algorithm used and on how well the resolution blurring is modeled (i.e. The $P$ matrix of equation 1.8 ) in the reconstruction and on the $\beta$ parameter of equation 1.12 when MAP reconstruction is used. The scanner's true intrinsic resolution can be obtained by setting $a$ equal to unity and $s$ equal to zero. For the Inveon the factors $d$ and $D$ are 1.59 and $161 \mathrm{~mm}$ as described in Section 1.6. Images reconstructed with 2D FBP can reach a $1.5 \mathrm{~mm}$ FWHM resolution at the center of the field of view for the Inveon as measured with ${ }^{18} \mathrm{~F}$ (Constantinescu and Mukherjee 2009). The factor $a$ is approximately equal to 1.2 for FBP (Lecomte 2004) and 
the effective Gaussian FWHM positron range for ${ }^{18} \mathrm{~F}$ is $0.54 \mathrm{~mm}$ (Lecomte 2004); note that this value differs from the mean value shown in Table 1.1. Using equation 1.14 the estimated PMT position decoding accuracy and the intrinsic resolution of the Inveon are estimated as 0.71 and $1.12 \mathrm{~mm}$ respectively. If $a$ is approximated as 1.1 for the OSEM3D/MAP reconstruction with $\beta=0.5$, the reconstructed image resolution with ${ }^{18} \mathrm{~F}$ is estimated as $1.4 \mathrm{~mm}$ FWHM.

\subsection{The Partial Volume Effect in Cardiac PET Imaging}

The heart is responsible for pumping blood throughout the body and thus has high energy demands. The left ventricle (LV) is the chamber of the heart responsible for pumping oxygenated blood throughout the systemic circulation. Thus, PET radiotracers designed to study perfusion and metabolism tend to accumulate in the LV myocardium and the corresponding PET images can be used to assess heart disease and myocardial viability (Moore and El Fakhri 2007). For example, $\left[{ }^{18} \mathrm{~F}\right]-\mathrm{FDG}$ is designed such that the corresponding activity distribution is related to glucose uptake (Taegtmeyer 1996) which can be used clinically to assess myocardial viability (Moore and El Fakhri 2007).

The LV myocardial wall thickness of mice is on the order of small-animal PET system spatial resolution. The reconstructed image spatial resolution of the Inveon's OSEM3D/MAP reconstruction is approximately $1.4 \mathrm{~mm}$ (Section 1.10 ) and LV myocardial wall thickness of mice is on the order of $1.0 \mathrm{~mm}$. Therefore, reconstructed image resolution does not allow the LV myocardium to be fully resolved and this can lead to inaccuracies in the quantification of myocardial activity (Hoffman et al. 1979). 
Consider the FDG-like activity distribution illustrated in Figure 1.4 on a transverse cross section through the MOuse BodY (MOBY) phantom (Segars et al. 2004) at the enddiastolic cardiac contraction phase; i.e. when the heart is in its final relaxed phase in contrast to end-systole where the heart is in the final contraction phase.

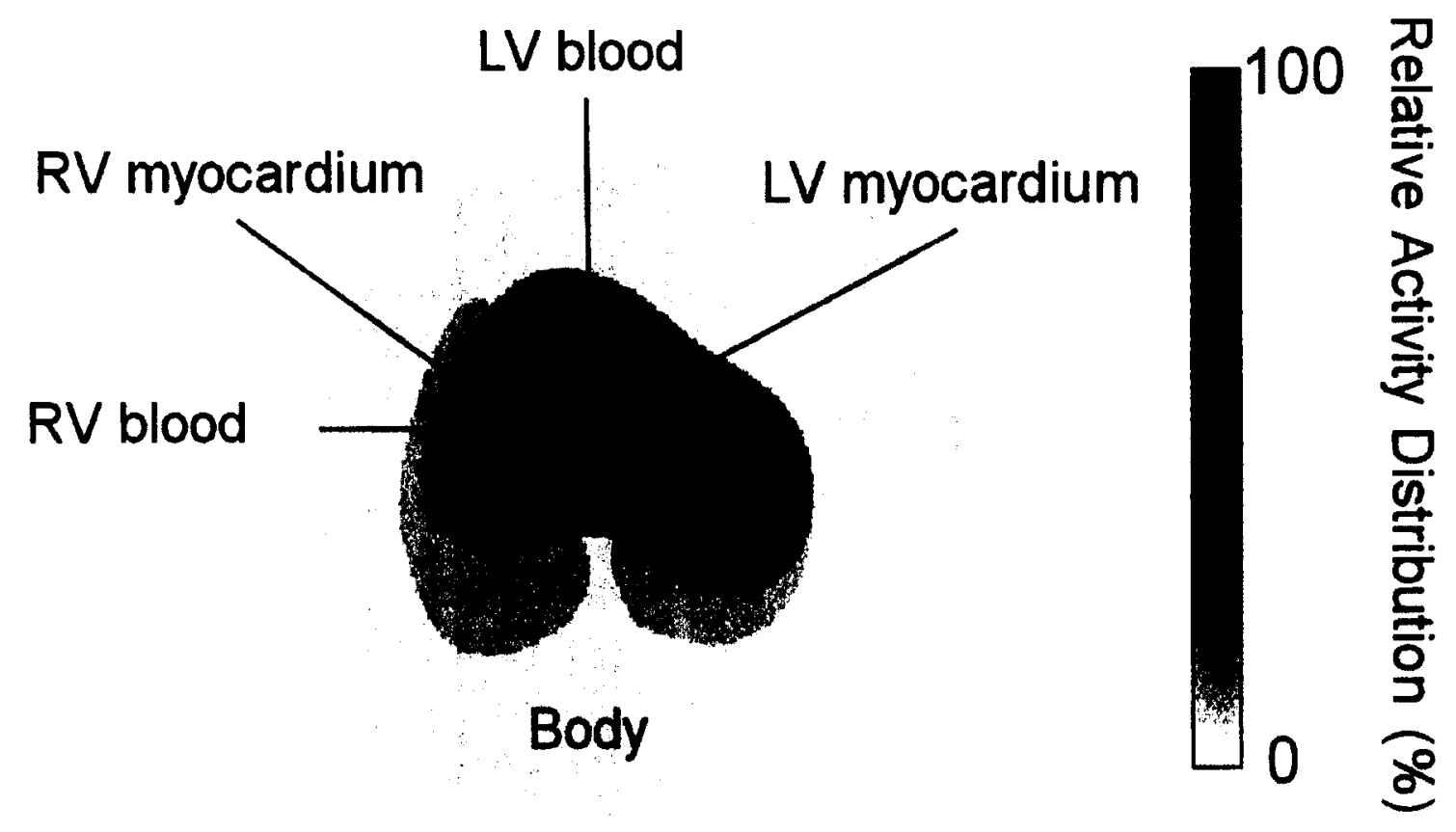

Figure 1.4. Relative activity distribution through a transverse slice of the MOBY phantom with the $L V$ and $R V$ myocardium having the highest relative activity.

The MOBY phantom is a digital phantom representing the mouse anatomy that includes cardiac and respiratory motion and allows input of individual organ activity. On 
the figure, the LV myocardium and right ventricle (RV) myocardium, the muscle responsible for pumping deoxygenated blood through the lungs, have a higher relative activity in comparison to the blood, liver, lung, and body. Each organ has homogeneous activity. To illustrate the effect that PET image resolution has on the activity distribution of Figure 1.4, Figure 1.5 displays the true activity distribution $\left(A_{\text {True }}\right)$, and a simulated measured PET activity distribution $\left(A_{P E T}\right)$ obtained by convolving $A_{\text {True }}$ with a $2 \mathrm{D}$ Gaussian point spread function (PSF) with a FWHM resolution of $1.4 \mathrm{~mm}$. The Gaussian convolution models the response of the scanner to an activity distribution placed within the field of view. The figure illustrates the effect of resolution degradation for the LV myocardium ( $\left.A_{L V, \text { myocardium }}\right)$, the blood/background ( $\left.A_{\text {blood/background }}\right)$ and the entire activity distribution ( $\left.A_{\text {rotal }}\right)$.

It is evident from Figure 1.5 that finite spatial resolution has the effect of blurring the measured activity distribution. In the case of the LV myocardium, the recovery coefficient $(\mathrm{RC})$, defined as the ratio of the measured peak activity to the true activity is $0.53 \pm 0.07$. The $\mathrm{RC}$ was sampled as the maximum values from the center of mass of the myocardium in $10^{\circ}$ increments. The $\mathrm{RC}$ decreases because the activity within the $\mathrm{LV}$ myocardium spills over into neighbouring pixels. The spillover effect is evident from $A_{P E T}$ and the profiles taken across the LV myocardium. Although the total activity is conserved in the image, the apparent recovery is decreased within the $\mathrm{LV}$ region. The magnitude of blurring is dependent on the surrounding activity distribution and the thickness of the myocardial wall, which explains why the homogeneity of the activity distribution was compromised, as indicated by a non-zero standard deviation. 


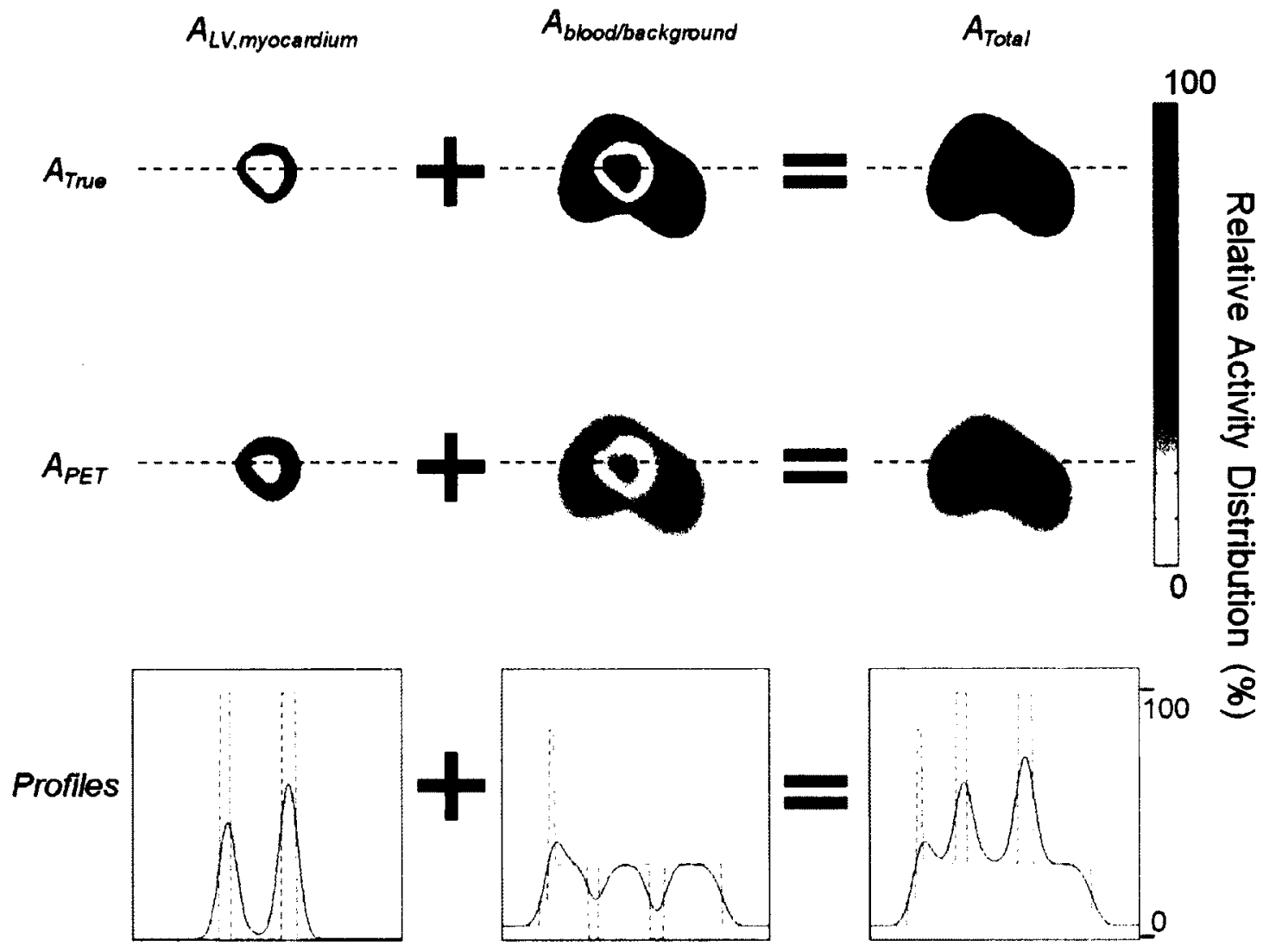

Figure 1.5. The effect of resolution degradation is illustrated with an FDG-like organ distribution on the cross section of the MOBY phantom. The top row shows the true activity distribution. The middle row displays the simulated measured PET images and the bottom row displays profiles across the activity distributions, with the location of each profile indicated on each image by the dashed line. The first, second and last columns show the relative activity distributions for the $L V$ myocardium, blood/background and total body respectively. The dashed profiles represent $A_{\text {True }}$ while the darker profile represents $A_{\text {PET }}$. 
Figure 1.6 demonstrates the effect of wall thickness on the RC for a FWHM Gaussian resolution of $1.4 \mathrm{~mm}$. The figure reveals a nonlinear relationship between RC and wall thickness. Furthermore, when the wall thickness is on the order of the resolution, there are significant losses. To fully recover the true activity value, the wall thickness must be greater than $2 \times$ FWHM. Thus, very small wall thicknesses suffer dramatic losses making it most difficult to quantify activity in these structures.

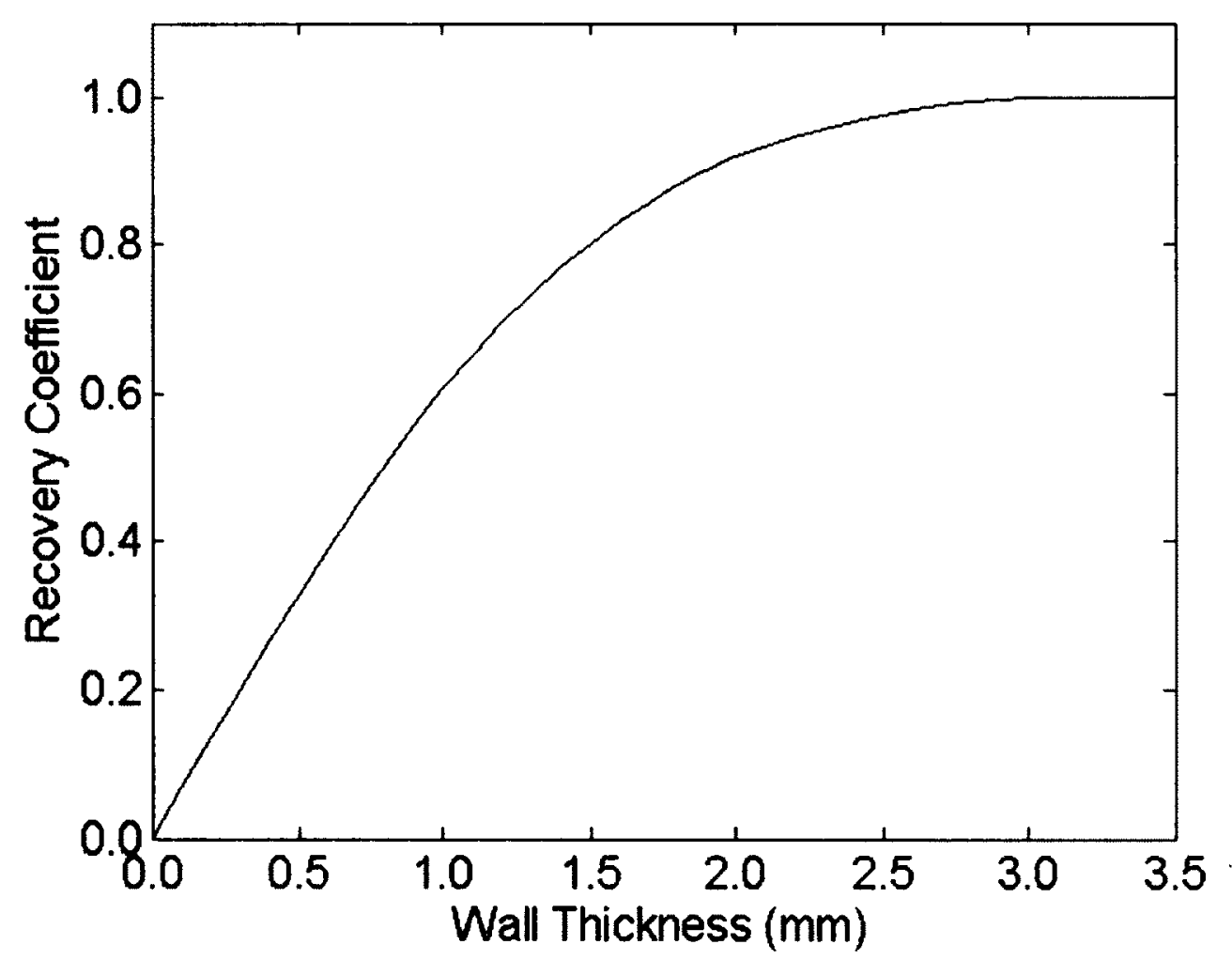

Figure 1.6. RC versus wall thickness for a FWHM Gaussian resolution of $1.4 \mathrm{~mm}$. 
The second column of Figure 1.5 demonstrates the effect that blood/background activity has on LV myocardium quantification. In this case, the LV myocardial activity is equal to zero, and thus no counts are expected to be recorded within the LV myocardial region. The image of $A_{P E T}$ and the profiles across the images indicate that this is not the case, and that counts from the blood/background activity distributions are in fact spilling into the LV region. The spill-in of the additional counts causes the apparent RC within this region to increase. The third column displays the images and profiles for the $\left(A_{\text {Total }}\right)$. The RC for the total activity distribution of $A_{P E T}$ was calculated as $0.68 \pm 0.04$. Thus, in this case, background activity increases the apparent RC and increases homogeneity of the activity distribution in the LV myocardium region. Therefore, each pixel in the simulated $\left(A_{\text {Total }}\right)$ contains a mixing of tissue signals from adjacent organs due to resolution blurring. The spatial mixing of tissue signals throughout the image is referred to as the partial volume (PV) effect. PV blurring affects quantitative accuracy and impedes the ability to localize individual organs because of spatial blurring.

The discussion thus far has been restricted to the 2D case. However, PET is a $3 \mathrm{D}$ imaging modality. Therefore, PV effects are observed in all spatial dimensions. Figure 1.7 illustrates $A_{\text {True }}$ and $A_{P E T}$ for a 3D LV myocardium with homogeneous activity distribution. The background activity was set equal to zero to eliminate spill in effects into the LV region. $A_{P E T}$ was computed by convolving $A_{\text {True }}$ with a 3D Gaussian PSF with an isotropic FWHM resolution of $1.4 \mathrm{~mm}$. The figure displays a cross sectional view (transverse), a front view (coronal) and a side view (sagittal) through the heart image. In this case the long axis of the heart is oriented along the $z$-dimension while the short axis of the heart is aligned with $(x, y)$. 

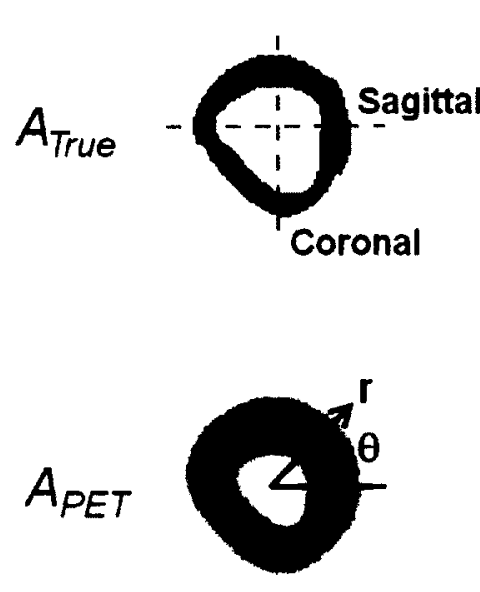

Transverse
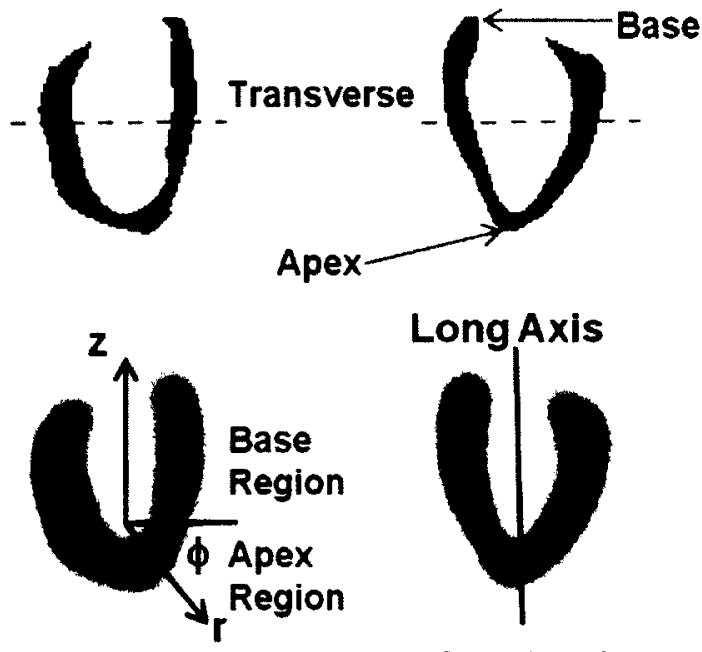

Coronal

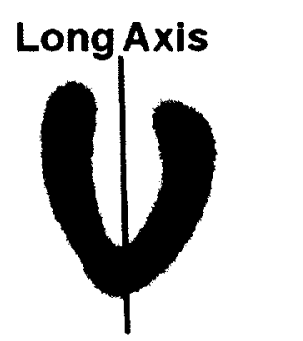

Sagittal

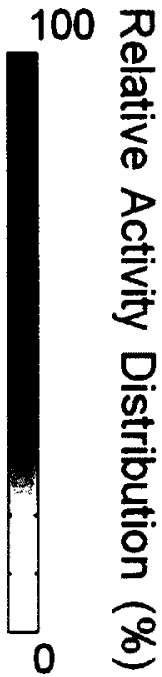

Figure 1.7. The 3D view of $A_{\text {True }}$ and $A_{P E Y}$ of the $\mathrm{LV}$ at end-diastole from the MOBY phantom. The locations of the transverse, coronal and sagittal views are located on $A_{\text {True }}$ and are denoted by dashed lines on the corresponding images. In this case, the LV long axis is oriented along the $z$ dimension, while the short axis is in $(x, y)$. The base and apex of the heart are indicated on the sagittal view of $A_{P E T}$. Sampling directions for polar map formation are indicated on $A_{P E T}$. A color map is shown on the right to give the relative activity distributions of the images.

PV effects can be observed on $A_{P E T}$ through the entire 3D structure. Between the apex (bottom) and base (top) of the heart, there is the least amount of PV losses because surrounding activity along the axis of the heart spills into the region partially compensating for the lost counts. At the base (top) and apex (bottom) limits of the heart, apparent PV losses are greater because blurring occurs in all three dimensions and there 
is no local activity along the $\mathrm{z}$ dimension to help compensate for these losses. It can be difficult to visualize the entire 3D activity distribution through the LV myocardium using the 3 views presented in Figure 1.7. Instead, it is customary to represent the 3D activity distribution using a polar map which samples the LV myocardial activity values and presents them as a $2 \mathrm{D}$ polar image as illustrated in Figure 1.8. The polar maps are generated by sampling the peak myocardial activity along a radial profile from the center of the LV myocardium. In the base region of the heart, the values are sampled in cylindrical coordinates, while in the apex region, the values are sampled in spherical coordinates. The sampling is indicated on the heart images of Figure 1.7 and on the polar maps in Figure 1.8. The figure illustrates the ideal polar map created from $A_{\text {True }}$ and the measured polar map generated from the image of $A_{P E T}$.

The ideal polar map of Figure 1.8 is expected with a homogeneous activity distribution and no resolution losses while the measured polar map reveals the PV effects due to finite resolution. Reductions in relative activity and non-uniformity are visible on the figure confirming the results from $A_{P E T}$ in Figure 1.7. These results demonstrate the need for a PV correction algorithm to restore quantitative accuracy of PET image myocardium activity distribution. While the discussion in this section is limited to PV effects resulting from a finite spatial resolution, cardiac and respiratory motion can also induce additional PV blurring effects. Motion due to cardiac contraction or lung inspiration/expiration causes additional blurring of the LV myocardium activity distribution that can reduce apparent uptake with FDG imaging. These motion effects further complicate quantitative accuracy and must be considered. 

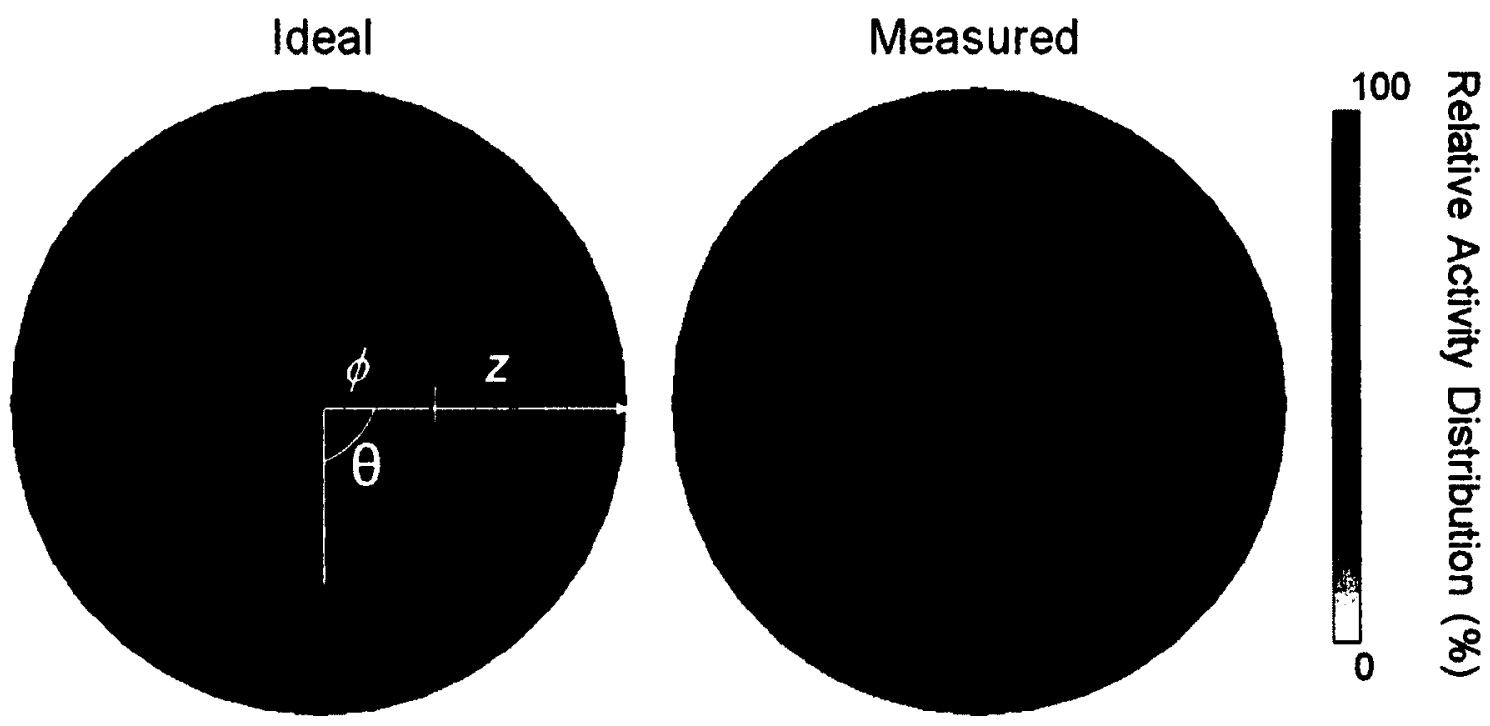

Figure 1.8. Polar maps for a 3D activity distribution in the $L V$ myocardium. The left image shows a uniform polar map for an LV myocardial activity distribution that does not suffer from PV losses, while the right image demonstrates a measured PET image that suffers from non-uniform PV effects. Polar map sampling is indicated on the ideal activity distribution. A color map is shown on the right, which gives the relative activity distribution of both polar maps. Polar maps are generated using FlowQuant (Klein et al. 2010).

\subsection{Literature Survey}

The PV effect in PET has been the focus of several research studies and various methods have been proposed to correct for this phenomenon (Rousset et al. 2007), however none are currently adopted as the standard. The correction methods can be divided into two subcategories: reconstruction-based methods and post-reconstruction image-based methods (Rousset et al. 2007). Reconstruction-based methods are performed 
by using prior anatomic information or by modeling the scanner's detector response (e.g. equation 1.8) to reduce PV effects in reconstructed PET images. Baete et al. (2004) use coregistered anatomic data derived from MRI in their iterative reconstruction algorithm. The anatomic data help improve the localization of cortical brain activity and are shown to help in the detection of subtle hypo-metabolic regions. Incorporating a detector response model into reconstruction algorithms can help reduce PV losses. Qi et al. (1998a, 1998b) used an explicit model of detector blur (equation 1.8) computed from Monte Carlo simulations of the specific detection system, as part of reconstruction. In contrast, Sureau et al. (2008) and Panin et al. (2006) incorporated physical point source measurements into their reconstruction to account for detector response effects. These resolution modeling approaches improve contrast recovery and reduce PV effects (Panin et al. 2006, Qi et al. 1998a, 1998b, Sureau et al. 2008) especially in comparison to analytic reconstruction algorithms such as FBP (Chatziioannou et al. 2000). However, these algorithms lack the ability to completely remove all PV effects. Therefore, additional PV correction is still required to recover accurate activity values in cardiac PET.

While reconstruction-based PV correction methods have received some attention, there is much more focus dedicated to post-reconstruction image-based methods. One of the simplest approaches to correct for the PV effect is to derive RCs from reconstructed images of phantoms with known activity concentrations and dimensions that resemble the organs of interest (Hoffmann et al. 1979). For example, this method can be used for myocardial PET imaging. deKemp et al. (2000) derive a global RC using a cardiac phantom measurement. The disadvantage with this type of approach is that it does not 
account for local variations in myocardial wall thickness or account for local variations in the myocardium/background activity distribution. Johnson et al. (2010) propose to correct for PV losses in myocardial PET imaging by first gating the PET image and correcting the measured end-diastolic activity concentration relative to the end-systolic image by assuming that the end-systolic image have full recovery. Using the isotope of interest a phantom measurement is then used to determine PV losses for the assumed systolic wall thickness. The advantage with this approach is that the positron range of the studied isotope is considered, and PV effects due to cardiac motion are minimized. However, this method still requires an $\mathrm{RC}$ measurement based on an assumed uniform wall thickness. Nuyts et al. (1996) propose an algorithm that convolves an anatomic heart image with the scanner PSF to derive regional RCs. Epicardial/endocardial contours are derived from the PET image using an automated edge detection program that is based on examining gradients across the myocardium (Nuyts et al. 1989). The contours are arranged to form a heart image, again assuming a uniform myocardial wall thickness, which is convolved with a PSF to derive the RCs. The disadvantage with the algorithm is that because a constant LV wall thickness is assumed, the algorithm could not consider variations in wall thickness, which limit quantitative accuracy in hearts with variable wall thickness. Gambhir (1990) and Porenta et al. (1995) performed PV correction by representing a profile across the myocardium of an ECG gated PET image as a onedimensional (1D) square wave convolved with a 1D Gaussian. The square wave has background, blood and myocardial activities which are separated by endocardial and epicardial wall borders, while the 1D Gaussian represents the resolution blur of the scanner. Each profile is fit using the method of nonlinear least squares. The algorithm 
eliminates PV effects due to motion using ECG gating and adopts to variable LV wall thicknesses. The model can provide an estimate to the 3D activity distribution of the $\mathrm{LV}$ myocardium. However, the model assumes that there is homogeneous activity across a radial profile of the $\mathrm{LV}$ myocardium and that $3 \mathrm{D}$ blurring can be described with $1 \mathrm{D}$ fitting. An alternative method for myocardial PET imaging by Muzic et al. (1998) expands on the ROI method by Huesman (1984). The algorithm examines an ROI in sinogram space which is projected from image space and includes modeling of scatter and resolution. The algorithm is capable of producing regional activity concentrations to within $5 \%$ of the truth when wall thicknesses are $1 \mathrm{~cm}$ or thicker in humans. However, the methodology has only been considered using 2D images/sinograms.

Teo et al. (2007) and Tohka et al. (2008) explore an iterative deconvolution approach that utilizes local image-based PSF measurements to reverse the blurring process in tumor and brain imaging respectively. These methods are beneficial because they do not rely on anatomical imaging. However deconvolution is shown to amplify noise in a corrected image (Teo et al. 2007, Tohka et al. 2008). Kirov et al. (2008) propose an iterative deconvolution technique that is regularized based on the local topology of the reconstructed image and using a PSF that is more smoothly varying than the true scanner PSF. The method simultaneously improves corrected image variance and recovery with regards to tumor imaging.

Other post-reconstruction image-based PV correction methods focus on incorporating additional parameters into kinetic models and several techniques are available. lida et al. (1988) include parameters into the kinetic model for $\left[{ }^{15} \mathrm{O}\right]$-water to account for tissue and blood spillover effects. The spillover from the blood pool into the 
myocardium is measured separately with a blood volume measurement using $\left[{ }^{15} \mathrm{O}\right]-$ carbon monoxide. Measuring spillover in this manner can lead to errors due to misregistration between the two images (Huang et al. 1985). Bergmann et al. (1989) perform a similar technique, but their model does not require a separate blood volume measurement. Hutchins et al. (1992), use a simplified approach that couples the blood pool spillover and myocardial tissue contribution to the PET signal into one parameter. Their model was tested on $\left[{ }^{13} \mathrm{~N}\right]$-ammonia imaging. Feng et al. (1996) apply a comparable technique, but examine cardiac FDG PET imaging. Bacharach et al. (2000) and Lodge et al. (2000) include PV correction parameters in their kinetic models of tumor blood flow. Incorporating PV correction factors into kinetic models is advantageous because the magnitude of the effect is constrained by the kinetic model. Furthermore, dynamic data are used so there are many image frames to derive the factors from, thus improving the robustness of the estimate (Soret et al. 2007). However, acquiring dynamic data can also be disadvantageous because the data are susceptible to body motion artifacts and the data are split into multiple time frames, thus decreasing the SNR of the activity distribution in the separate image frames. The discussed models also ignore spill-in from all adjacent organs (RV blood spillover is included) into the tissue of interest, thus potentially biasing the results.

Anatomic imaging, from modalities such as CT and MRI, can offer valuable information for PV correction (Rousset et al. 2007). Transmission imaging, as proposed by lida et al. (1991), can be combined with a $\left[{ }^{15} \mathrm{O}\right]-\mathrm{CO}$ blood pool scan to derive an extravascular density image which describes the fractional volume containing myocardium tissue. This image can be used as an indicator of viable myocardium. 
Wassenaar et al. (2006) use a similar technique but focus on explicitly correcting PV effects of the myocardial activity. The method performs well for 3D PET over a range of wall thicknesses, but is most sensitive to errors in the smaller wall thicknesses, potentially because of the increased effect of background spillover into the myocardium. The partition based method that is used for brain imaging (Müller-Gärtner et al. 1992), requires a segmented MRI image that is registered with a PET image. The MRI image is segmented into gray matter, white matter, and cerebrospinal fluid images where each tissue type is assumed to have uniform uptake. The activity of each tissue is adjusted to match the actual contribution to the PET image by convolving the segmented images with the PSF and comparing to the PET image. Meltzer et al. (1996) expanded on this method by increasing the number of tissue segments to four. The geometric transfer matrix (GTM) method (Rousset et al. 1998), involves acquiring a high resolution anatomical MRI image of the brain that is coregistered to a PET image. MRI images are segmented into $N$ ROIs, where each ROI represents a true tissue segment that is assumed to have homogeneous radioactivity. The activity in each segment is then determined by comparing the PET image to a linear combination of the segments. The multiresolution approach by Bouisson et al (2006) decomposes a high resolution image (e.g. MRI or CT) and a low resolution image (e.g. PET) using wavelets and incorporates spatial frequency information from the high resolution image into the low resolution image to correct for PV effects. In the case of cardiac imaging, respiratory and cardiac motion further complicate coregistration in the combined modalities of PET/CT (Alessio et al. 2010a) and PET/MRI (Nekolla et al. 2009). The accuracy of anatomic imaging based PV correction techniques depends on the ability to segment and coregister the anatomic 
image to the PET image (Rousset et al. 2007) and is thus a potential limitation of these methods.

There are limited studies on PV correction for cardiac mouse PET imaging. Green et al. $(1998,2004)$ derive a global RC using the method of Gambhir (1990) for ungated PET images. Fang and Muzic (2008) propose a method for correction of the imagederived blood input function in mice for spillover and partial volume losses, but the PV correction of myocardial activity is not explicitly considered. In a recent study, Maroy et al. (2010) obtain PV free estimates of time activity curves of various organs from PET images of mice. Organ segmentation is performed using the local means analysis method which is a segmentation method based on local differences in tracer kinetics (Maroy et al. 2008). Each segmented organ is assumed to have homogeneous activity, and PV effects are minimized by sampling only a subset of voxels from each segmented organ that are minimally affected by PV losses. Their method did not require PSF measurement but assumed each organ, including the heart had homogeneous activity. This method did not consider the LV myocardium explicitly.

The GTM method can restore quantitative accuracy to within $10 \%$ in brain imaging (Roussett et al. 1998), while iterative deconvolution techniques can provide activity estimates that are approximately within $10 \%$ of the true activity for tumour imaging (Teo et al. 2007). As discussed in Section 1.11 PV effects decrease the relative activity distribution in cardiac mouse PET imaging by approximately $30 \%$. The goal of this research is to create a novel PV correction technique to restore quantitative accuracy in cardiac mouse PET imaging to within $10 \%$. It is predicted that this level of accuracy can be obtained using a PV correction method that is based on convolving an estimated 
LV image with the scanner PSF. Estimated LV images are created using five parameters including blood, background and myocardial activities that are separated into distinct compartments by an endocardial wall border and a myocardial wall thickness. In Chapter 2, a combined 1D/2D method is developed. Blood and background activities along with the myocardial wall border positions are obtained using 1D fitting. A 2D model is created using the fitted parameters to derive the myocardial activity. In Chapter 3 , the $1 \mathrm{D} / 2 \mathrm{D}$ method is experimentally validated and the $1 \mathrm{D} / 2 \mathrm{D}$ model is expanded to $1 \mathrm{D} / 3 \mathrm{D}$ to take into consideration the entire 3D image volume. Finally, a fully $3 \mathrm{D}$ model is explored in Chapter 4 where parameters are fit in 3D such that no $1 \mathrm{D}$ model is required. 


\section{Chapter 2. \\ A Partial Volume Correction Strategy for Cardiac Mouse PET Imaging using a 1D/2D Method}

Preliminary results for the work presented in this chapter were published in abstract form (Dumouchel and deKemp 2008, 2011a).

(C) 2011 IEEE. Reprinted with permission, from Tyler Dumouchel and Robert A. deKemp, Analytical-Based Partial Volume Recovery in Mouse Heart Imaging, IEEE Transactions on Nuclear Science, February 2011 (Dumouchel and deKemp 2011 b). This paper has been reformatted and the introduction has been edited to conform to the thesis format.

\subsection{Introduction}

In this chapter, a PV correction method for cardiac mouse PET imaging is explored that uses a 1D model to obtain myocardium contours and surrounding activity values. The $1 \mathrm{D}$ information is interpolated onto a $2 \mathrm{D}$ grid and a $2 \mathrm{D}$ filter is applied to determine RCs for myocardial activity. The $1 \mathrm{D}$ model is rooted on the basis of convolving a ID square wave model with a 1D Gaussian scanner PSF (Gambhir 1990). The 1D method has been used to evaluate LV geometry and contractile function in 
humans (Porenta et al. 1995, Freiberg et al. 2004, Khorsand et al. 2003, Bartlett et al. 1996) and is also a potential solution to the PV problem for humans (Gambhir 1990). Green et al. $(1998,2004)$ also used this method to derive global RCs applied to static (non ECG gated) mouse images. The limitation with the 1D methodology is that it assumes that there are PV losses only along the profile dimension. PET is a 3D imaging modality and is subject to blurring along all spatial dimensions, and thus the $1 \mathrm{D}$ model might not be completely accurate at describing the blurring process. However, using the ID model, the myocardial borders, blood activity and background activity can be determined in a computationally efficient manner.

In this study, the fitted 1D parameters are arranged onto a 2D grid in order to increase correction accuracy of the myocardial activity, by taking into account additional PV losses due to the local curvature of the myocardium. The blood, background and contour information are interpolated onto a 2D grid and the scanner PSF is utilized to derive regional RCs for PV correction of PET images. The model is robust in the setting of variable blood and background activities and image noise. Furthermore, there is no requirement for a secondary anatomic image and thus coregistration errors do not occur. The methodology works independently of a tracer kinetic model to estimate the LV geometry, and accounts for cardiac motion by using ECG gating, in an attempt to eliminate the major factors affecting cardiac mouse image quantification.

The objective of this chapter is to present the framework for the proposed 1D/2D correction and demonstrate how the algorithm provides a more accurate estimate of the true myocardial activity than the raw PET image or a $1 \mathrm{D}$ model alone. The 1D/2D model is characterized in a variety of situations to validate the algorithm's performance when 
noise, different background activities, variable wall thicknesses and wall motions are encountered. This chapter focuses on mouse imaging, but the methodology could be adapted for other species with similar relationships between resolution and heart size.

\subsection{Materials and Methods}

\subsubsection{The ID/2D Partial Volume Correction Algorithm}

The PV correction methodology is based on the assumption that the activity distribution along a radial profile through the LV myocardial wall can be described by a rectangular function having adjacent blood and background activities. The measured activity distribution is subject to resolution blur from the detection system and reconstruction process. In the heart wall this blurring is modeled by a $1 \mathrm{D}$ convolution operation between a rectangular (square) function and a Gaussian function (Gambhir 1990, Porenta et al. 1995). The operation and all parameters are shown in Figure 2.1. A radial profile across a PET image $\left(I_{P E T}\right)$ is thus described by:

$$
\begin{aligned}
\frac{1}{2} C_{g}+\frac{1}{2} C_{b}+ & \frac{1}{2} C_{m} \operatorname{erf}\left(\frac{\mathrm{x}-\mathrm{R}}{\sqrt{2} \sigma}\right)-\frac{1}{2} C_{m} \operatorname{erf}\left(\frac{\mathrm{x}-\mathrm{R}-\mathrm{w}}{\sqrt{2} \sigma}\right) \\
- & \frac{1}{2} C_{b} \operatorname{erf}\left(\frac{\mathrm{x}-\mathrm{R}}{\sqrt{2} \sigma}\right)+\frac{1}{2} C_{g} \operatorname{erf}\left(\frac{\mathrm{x}-\mathrm{R}-\mathrm{w}}{\sqrt{2} \sigma}\right)
\end{aligned}
$$

There are five free parameters in the 1D analytic model including $C_{m}(\theta, g)$, $C_{b}(\theta, g), C_{g}(\theta, g), R(\theta, g)$ and $w(\theta, g)$. The scanner PSF is characterized by $\sigma$, and measured as described in Section 2.2.3. The $\theta$ and $g$ variables are used to explicitly indicate that the quantities are a function of angle and gate respectively. The assumption that a profile through the heart wall can be described by a rectangular function requires that the images 
used for fitting are motion-free, and must thus be ECG-gated. The 1D model is assumed to accurately predict $w(\theta, g), R(\theta, g), C_{g}(\theta, g)$ and $C_{b}(\theta, g)$ locally and this information can be used to develop a 2D model to estimate the myocardial activity in a given image plane.
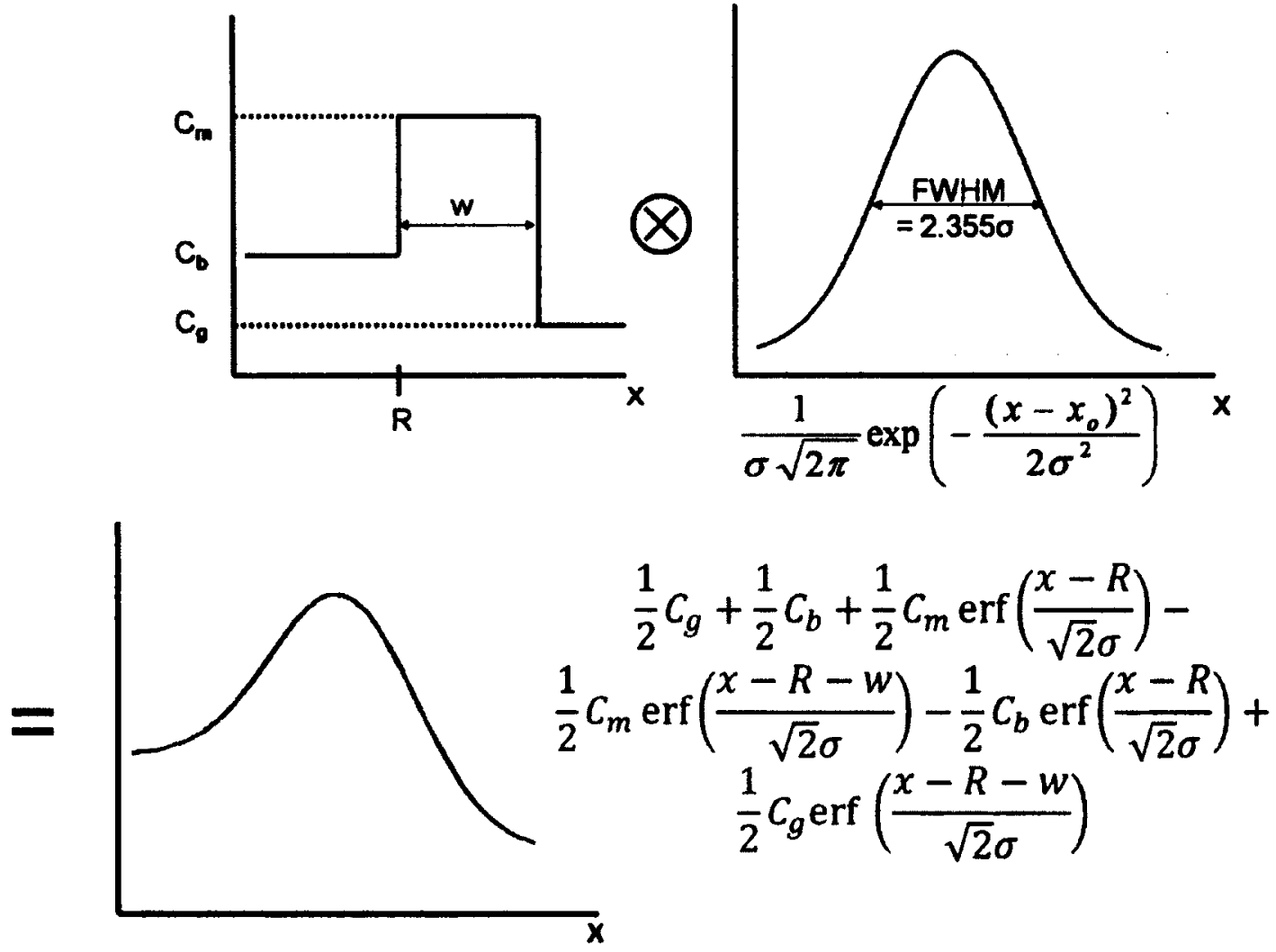

Figure 2.1. Illustration of the $1 \mathrm{D}$ convolution used to approximate the PV effects in the myocardium. $C_{m}$ is the true myocardial activity, $C_{b}$ is the blood activity, $C_{g}$ is the background activity, $R$ defines the location of the myocardial/blood border, $w$ is the width of the LV myocardium, $\sigma$ defines the width of the Gaussian (FWHM = 2.3550), erf is the error function, $\otimes$ is the 1D convolution operator and $x$ is the radial position along the profile. $\theta$ and $g$ denote that the values are obtained as a function of radial angle and gate respectively. 
The combined 1D/2D model is based on interpolating $w$ and $R$ onto a 2D Cartesian grid and creating two images. One set of gated images consists of the myocardial contours filled in with a true relative activity value of unity, $I_{m y o}(g)$, and the second set of gated images are composed of the blood and the background information, $I_{\text {back }}(g)$ and $I_{\text {blood }}(g)$, as determined from $C_{b}(\theta, g)$ and $C_{g}(\theta, g)$. Making use of the distributive property of convolution, the apparent activity in the myocardium in the PET image can be described using the following equation:

$$
\begin{gathered}
I_{P E T, \text { myo }}(g)=I_{P E T}(g)- \\
{\left[I_{b a c k}(g)+I_{b l o o d}(g)\right] \otimes P S F}
\end{gathered}
$$

where $I_{P E T}(g)$ is a gated PET image, PSF is the point spread function, $\otimes$ is the 2D convolution operator and $I_{P E T, m y o}(g)$ is an estimate of the apparent myocardial activity in the gated PET image in the absence of blood and background spillover. The estimated myocardial image, $I_{m y o}(g)$, can be convolved with the PSF to obtain an estimate of the regional RCs to correct the PET image by the following equations:

$$
\begin{gathered}
I_{\text {conv,myo }}(g)=I_{\text {myo }}(g) \otimes P S F \\
C_{m, 2 D}(\theta, g)=\frac{I_{P E T, m y o}\left(r=\operatorname{argmax}\left(I_{P E T, m y o}(\theta, g)\right), \theta, g\right)}{I_{\text {conv,myo }}\left(r=\operatorname{argmax}\left(I_{P E T, m y o}(\theta, g)\right), \theta, g\right)}
\end{gathered}
$$

where $I_{\text {conv,myo }}(g)$ is an image containing estimated regional RCs and $C_{m, 2 D}(\theta, g)$ is an estimate of the true myocardial activity sampled along a radial profile at an angle $\theta$. The ratio presented in equation 2.4 is computed at the maximum of the myocardial activity. The entire process is illustrated in Figure 2.2. Images in Figure 2.2 were cropped around the myocardium in order to emphasize the process visually. 

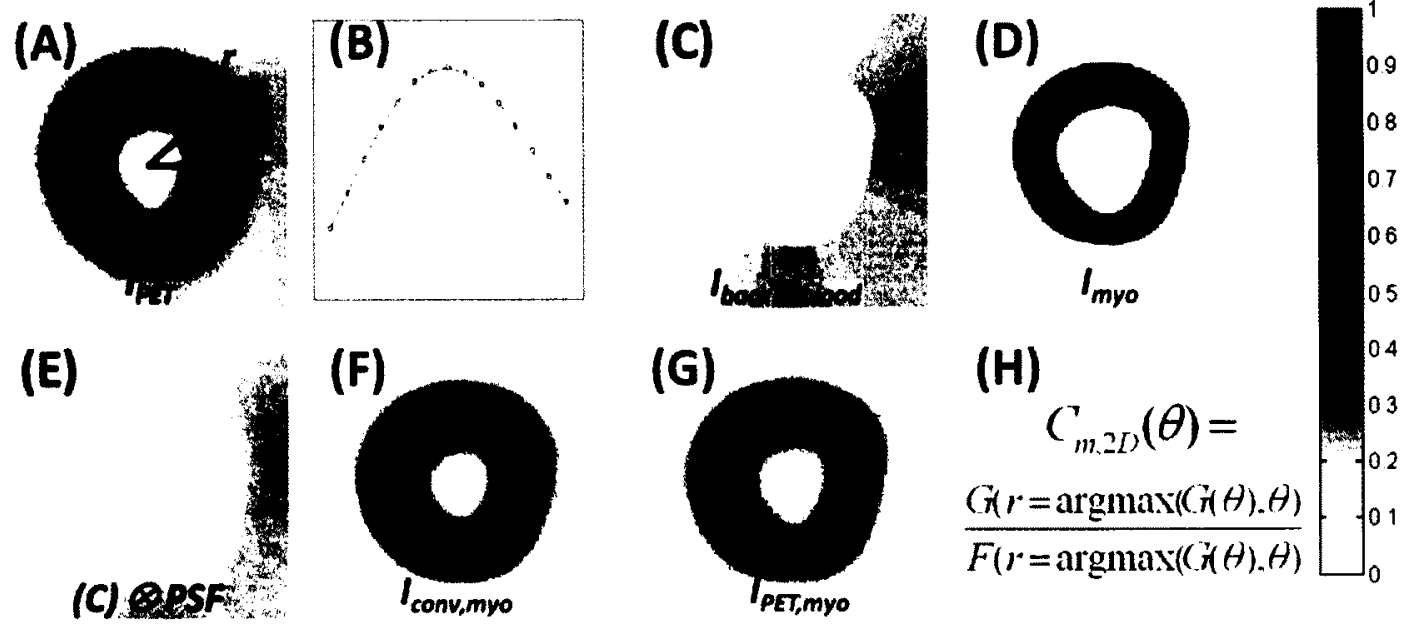

(E)

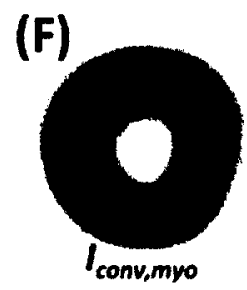

Figure 2.2. Illustration of the proposed PV correction process on a simulated MOBY phantom: (A) A gated short axis end-diastolic PET image of the LV myocardium was selected and profiles were taken across the image; one such profile is shown on the image. (B) Radial profiles (circles) were fit using the equation in Figure 2.1. The 1D fitted information, namely $w(\theta), R(\theta), C_{g}(\theta)$ and $C_{b}(\theta)$ were used to construct $(C)$ an estimated $2 D$ background and blood image and $(D)$ an estimated 2D heart image with uniform activity equal to unity. (E) and (F) represent the convolution of images $(C)$ and $(D)$ respectively with the PSF, thus (F) is an image of the regional RCs. (G) represents a transverse slice of the PET image (A) with blood and background removed. An estimate of the true activity in the myocardium can be obtained with the ratio $(H)$ sampled in the region of the myocardium along a profile angle $\theta$. This process was repeated for all gates (g).

The PET images are linearly interpolated onto a $0.1 \mathrm{~mm}$ pixel grid prior to performing any operations. The center of each image is found by summing over all gates and defining the center of mass of the LV cavity. Thirty-six radial profiles are obtained in 
$10^{\circ}$ increments for each gate. The maximum value of each profile is found and a $1.5 \mathrm{~mm}$ band around this value is selected for fitting. The selection of this band for fitting ensures that a non-uniform background or spillover from the contralateral wall has minimal bias on the 1D fitting results. All $1 \mathrm{D}$ model parameters are fit independently for each gate $(g)$ and angle $(\theta)$ using the method of nonlinear least squares. Nonlinear least squares is used because it is computationally efficient and is convergent. The blood activity, $C_{b}(\theta, g)$, is averaged over all angles to obtain one value for each gate, $C_{b}(g)$, to form the estimated blood image, $I_{\text {blood }}(g)$. The background activity is allowed to vary over angle and gate. The background images, $I_{b a c k}(g)$, are created by assigning the measured $C_{b}(\theta, g)$ values as pie slices originating from the center of the object outside the epicardial border. This can be visualized in the example of Figure 2.2(C). $I_{b a c k}(g)$ included enough background pixels (greater than $2 \times$ FWHM from epicardial borders) to avoid truncation effects from the convolution in equation 2.2. The myocardial activity, $C_{m, 2 D}(\theta, g)$, is sampled at the peak value as a function of angle and gate following equation 2.4. The goal of the proposed correction is to provide a single estimate of average myocardial activity in each angular region of the heart image. Thus, the final myocardial activity is found by averaging $C_{m, 2 D}(\theta, g)$ over all gates to obtain $C_{m, 2 D}(\theta)$

\subsubsection{PET System Description}

The system used in this study is the Inveon (Siemens Preclinical Solutions, Knoxville, TN, USA). This system is described in detail in Section 1.6. Briefly, data are acquired in list mode with a $350-650 \mathrm{keV}$ energy window and a $3.42 \mathrm{~ns}$ timing window. The raw data are binned directly into 3D sinograms using a ring difference of 79 and a span of 3. The OSEM3D/MAP algorithm is used for image reconstruction (Section 1.8) 
with 2 OSEM3D iterations, 18 MAP iterations, a $\beta$ of 0.05 and both fastMAPTM and uniform resolution enabled. The criteria for selecting a $\beta$ of 0.05 will be discussed in Section 2.3.1. Images are reconstructed onto a $128 \times 128$ or $256 \times 256$ matrix with 0.2 $\mathrm{mm}$ pixels in the transverse dimension and 159 axial planes with a slice thickness of 0.8 $\mathrm{mm}$. All images are corrected for random events by the delayed coincidence window method. Detector and geometry efficiencies are corrected as part of reconstruction.

\subsubsection{Point Spread Function}

The reconstructed PSF in the transverse plane was measured by fitting a Gaussian to the line response of the scanner. A polyethylene tube with a small inner diameter and outer diameter was selected for performing the measurement (PE-10 tubing, inner diameter $=0.28 \mathrm{~mm}$, outer diameter $=0.64 \mathrm{~mm}$ ). An $\left[{ }^{18} \mathrm{~F}\right]$-solution, with a concentration of $1900 \mathrm{MBq} / \mathrm{mL}$, was injected into the tubing and placed $3.5 \mathrm{~mm}$ from the axial center of the field of view. A one-hour acquisition was performed and reconstructed with standard settings. Alternate reconstructions were explored by changing the $\beta$ values in OSEM3D/MAP to study how resolution was affected by $\beta$. The central transverse plane and two outer planes, $\pm 4 \mathrm{~cm}$, were used for resolution determination. Profiles that were 15 pixels long were taken through the maximum intensity pixel in the tangential and radial directions for each slice. All profiles were fit to a $1 \mathrm{D}$ Gaussian function using the method of nonlinear least squares in MATLAB (The MathWorks, Inc., Natick, MA, USA). It was assumed that the PSF was Gaussian with radial and tangential resolutions given by the FWHMs of the fitted distributions. A Gaussian function is of particular interest because the $(x, y)$ smoothing generated by convolution is separable and it is necessary to have a Gaussian PSF for the 1D model fitting presented in Section 2.2.1. 
The goodness of fit was assessed using the chi-squared statistic. The results were summarized by plotting the average resolution \pm standard deviation (SD) over all fits versus the $\beta$ value.

\subsubsection{Noise Properties}

For a realistic PET simulation, it is important to model the noise properties of a reconstructed image accurately. To determine these properties for the Inveon OSEM3D/MAP reconstruction, a $30 \mathrm{~mL}$ syringe with a $2.4 \mathrm{~cm}$ outer diameter and thin plastic walls was filled with $22 \mathrm{~mL}$ of a $100 \mathrm{MBq}\left[{ }^{13} \mathrm{~N}\right]$-solution. A high initial concentration was used so that a wide range of count rates could be studied as the activity decayed. The syringe was centered in both the axial and transverse fields of view. A 100 minute acquisition (ten half-lives) was performed with standard acquisition parameters. The measured data were binned into 3D sinograms divided into ten-minute intervals. The sinograms were reconstructed with OSEM3D/MAP and standard settings onto a $128 \times$ 128 grid. A cylindrical volume of interest (VOI) was drawn on the central region of the syringe, $18.2 \mathrm{~mm}$ in diameter in the transverse plane and $4 \mathrm{~cm}$ in the axial direction. The distribution of pixel values was fit to a Gaussian function as shown previously for MAP (Qi and Leahy 2000). The standard deviation $\left(\sigma_{V O I}\right)$ and the mean $\left(\mu_{V O I}\right)$ were also calculated for each region. A simulated image was created to ensure the noise properties could be simulated accurately in comparison to a measured PET image of the cylinder. Gaussian noise was added to a circular image which was then convolved with the PSF. The simulated image had a final SNR of 12.5 , which was compared to a PET image with the same SNR. 
With the FBP reconstruction algorithm, the square of the SNR within each VOI in a uniform cylinder is proportional to the noise equivalent count (NEC) rate of the tomograph (Strother et al. 1990) according to the following equation:

$$
S N R^{2}=\left(\frac{\mu_{V O I}}{\sigma_{V O I}}\right)^{2} \propto N E C=\frac{T^{2}}{T+S+a f R}
$$

where $T, S$ and $R$ are the number of true, scattered and random events, respectively. The factor $f$ is the fraction of the sinogram width that the object subtends radially and the factor $a$ is between 1-2 depending on how random coincidences are corrected ( $a=2$ in this case for delayed coincidence randoms subtraction). The NEC can be approximated from the count rates stored in the header files (Dumouchel and deKemp 2008), assuming that the scatter fraction measured from the $2.5 \mathrm{~cm}$ diameter National Electrical Manufacturers Association (NEMA) mouse-like phantom (NEMA 2008) is applicable to the uniform syringe. This is true, as the syringe and the phantom have similar dimensions. A scatter fraction of $8.8 \%$ was adopted (Dumouchel and deKemp 2008). The SNR of each interval was plotted versus the NEC to observe if the SNR from an OSEM3D/MAP reconstruction scales linearly with the NEC. The SNR was also plotted versus the true coincidence counts, as this quantity can be measured for a given acquisition.

To estimate the noise present in a typical heart scan on the Inveon, a $30 \mathrm{~g}$ normal mouse was anesthetized under $2 \%$ isoflurane and injected with $30 \mathrm{MBq}$ of $\left[{ }^{18} \mathrm{~F}\right]$-FDG in the tail vein and a one-hour acquisition was performed with standard settings. Two time frames were reconstructed including a 4-5-minute frame and a 5-10-minute frame postinjection. Standard settings were used to reconstruct the images onto a $256 \times 256$ grid. A $100 \mathrm{~mm}^{3}$ VOI was drawn over the liver and the mean and standard deviation of the 
region were computed. The LV myocardium was subject to PV effects, and thus if a VOI was placed over the LV, the measured SNR would be biased because the activity distribution is known not to be uniform in this region. To determine the SNR in the heart, the SNR in the liver was scaled by the activity ratio assuming a linear relationship exists between square of the SNR and true activity.

\subsubsection{Simulations}

Gaussian noise was added to a 2D image, representing a short-axis slice of the heart, and convolved with the PSF. The simulations were constructed such that a range of wall thicknesses and wall motions was considered. Four digital phantoms were created, namely: (1) a concentric uniform wall thickness and wall motion phantom, (2) a phantom with variable wall thickness and uniform contraction, (3) a phantom with variable wall thicknesses including a thinned wall with no contraction representing infarcted (i.e. diseased or scar) tissue and (4) a cross section of the MOBY digital phantom. Phantoms 1-3 were constructed such that the end-diastolic myocardial wall thickness and endocardial wall motion were similar to the MOBY phantom while ejection fractions were estimated from a mouse LV quantification study (Stegger et al. 2009); ejection fraction is the fraction of total LV blood volume that is pumped out of the LV cavity on each heart beat and is defined as the percent difference in blood volume between enddiastole and end-systole. For these phantoms, there was no wall motion simulated within the time of each gate. A Fourier series was used to describe the endocardial wall motion as a function of gate $(g)$ :

$$
r=\frac{a_{0}}{2}+\sum_{n=1}^{2} a_{n} \cos \left(\frac{2 \pi n g}{N}\right)+\sum_{n=1}^{2} b_{n} \sin \left(\frac{2 \pi n g}{N}\right)
$$

where $r$ was defined as location of the endocardial wall from the center of the cavity, $a_{n}$ 
and $b_{n}$ were the Fourier coefficients, and $N$ was the number of gates which was set to 16 . The coefficients of the series were estimated by fitting endocardial contours from the MOBY phantom using the method of nonlinear least squares. All phantoms were created such that the endocardium contracted radially inward toward a defined centroid in 2D following equation 2.6, while conservation of the myocardial wall area was used to define the epicardial contour contraction in 2D. Phantom 1 had an ejection fraction of $64 \%$, simulating a healthy heart, an end-diastolic wall thickness of $1 \mathrm{~mm}$, and an endsystolic wall thickness of $1.3 \mathrm{~mm}$. Phantom 2 also had an ejection fraction of $64 \%$ but the wall thicknesses ranged from 0.4 to $1.4 \mathrm{~mm}$ in end-diastole and from 0.7 to $1.7 \mathrm{~mm}$ in end-systole. Both phantoms 1 and 2 contracted concentrically. Phantom 3 was constructed with an ejection fraction of $32 \%$ to simulate a heart with infarcted tissue (Steggar et al. 2009) in which there was a thin wall that did not thicken, but translated as the adjacent heart walls contracted. The wall thicknesses ranged from 0.2 to $1.6 \mathrm{~mm}$ in end-diastole and from 0.2 to $1.8 \mathrm{~mm}$ in end-systole. The end-diastolic and end-systolic phases of this phantom are illustrated in Figure 2.3(a) and 2.3(b), respectively. Phantom (2) is not shown, but has a similar appearance to Phantom (3). Phantom (4) was extracted from a short axis slice of a MOBY phantom simulation. The phantom was reoriented to align the heart along Cartesian coordinates, and the central short axis slice of the heart was selected.

To determine appropriate blood and background activity for the phantoms, five normal mice were anesthetized under $2 \%$ isoflurane and imaged using $\left[{ }^{18} \mathrm{~F}\right]$-FDG. The mice ranged in body mass from $30-50 \mathrm{~g}$ while the injected activity was between $30-70$ MBq. Two five-minute frames were reconstructed with OSEM3D/MAP at 5 and 55 
minutes post-injection. The images were reconstructed onto a square grid of 256 pixels with a pixel size of $0.2 \mathrm{~mm}$. VOIs were placed to encompass the organs of interest and the maximum value from each region was recorded. The maximum values were used to minimize the PV losses in smaller structures. Organs analyzed included the LV myocardium, LV blood pool, RV myocardium, RV blood pool, liver and background. The background VOI was a small region placed outside of the heart, adjacent to the LV lateral free wall. For the purpose of forming simulations, only the relative uptake with respect to the $\mathrm{LV}$ was important.
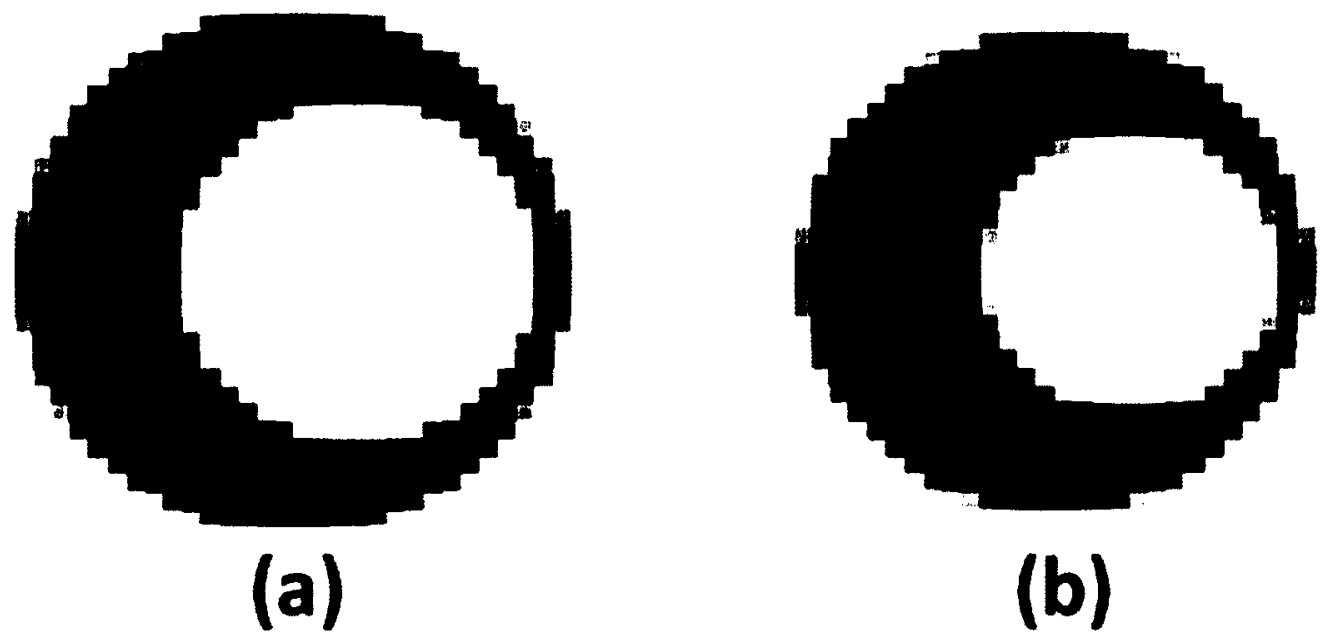

Figure 2.3. Illustration of Phantom 3 in (a) end-diastole and (b) end-systole. The wall thicknesses ranged from $0.2-1.6 \mathrm{~mm}$ and $0.2-1.8 \mathrm{~mm}$ in end-diastole and end-systole respectively. The ejection fraction was $32 \%$. Phantom 2 has a similar appearance but with concentric contraction.

Phantoms 1-3 were based on the measured values of LV myocardium, blood and liver activities. An LV blood activity of 0.01 was used in all cases because in the mice 
imaged it was observed that FDG clears from the blood rapidly after injection. The relative liver activity ranged from 0.02 to 0.41 taking into consideration two standard deviations from the mean value. For phantoms 1-3, three background regions were considered in this range, namely $0.02,0.22$ and 0.41 . To evaluate the effects of noise and wall thickness a representative background of 0.22 was considered. For phantom 4, two activity distributions were created using all FDG uptake values for both the early and late time frames.

Phantoms 1-3 were constructed on $0.01 \mathrm{~mm}$ pixels and downsampled to $0.2 \mathrm{~mm}$ in order to simulate the tissue fraction effect that occurs when the pixel size is too large to adequately sample the myocardium. The MOBY phantom was simulated directly onto $0.2 \mathrm{~mm}$ pixels. Gaussian distributed noise was added to all of the phantoms and a variety of noise levels were simulated as determined from the analysis of Section 2.2.4. Following the addition of noise, the PSF was applied through convolution to obtain simulated PET images.

The accuracy of the PV correction from the 1D/2D model was assessed by directly comparing the recovered value to the true myocardial activity. The mean and standard deviation were computed over the 36 sampled myocardial activity estimates, $C_{m, 2 D}(\theta)$, from the $1 \mathrm{D} / 2 \mathrm{D}$ model to obtain a global estimate $C_{m, 2 D} \pm \mathrm{SD}$. The $1 \mathrm{D}$ fitted values were averaged over all gates to obtain 36 myocardial estimates, $C_{m, I D}(\theta)$ for comparison to the $1 \mathrm{D} / 2 \mathrm{D}$ results. The mean and standard deviation were computed over the 36 estimates of $C_{m, I D}(\theta)$ to obtain $C_{m, l D} \pm \mathrm{SD}$. The entire algorithm was implemented in MATLAB. 


\subsection{Results}

\subsubsection{Point Spread Function}

Figure 2.4 demonstrates how the FWHM resolution changes with the $\beta$ value for a line source in air. Smaller $\beta$ values in the reconstruction inevitably lead to improved resolution. However, as the $\beta$ value decreases, the assumption of a Gaussian PSF becomes less accurate. At a $\beta<0.05$, the computed chi-squared values are above the critical value of 21.0 taken at a significance level of 0.05 . Table 2.1 illustrates how the average chi-squared value increases with a decreasing $\beta$.

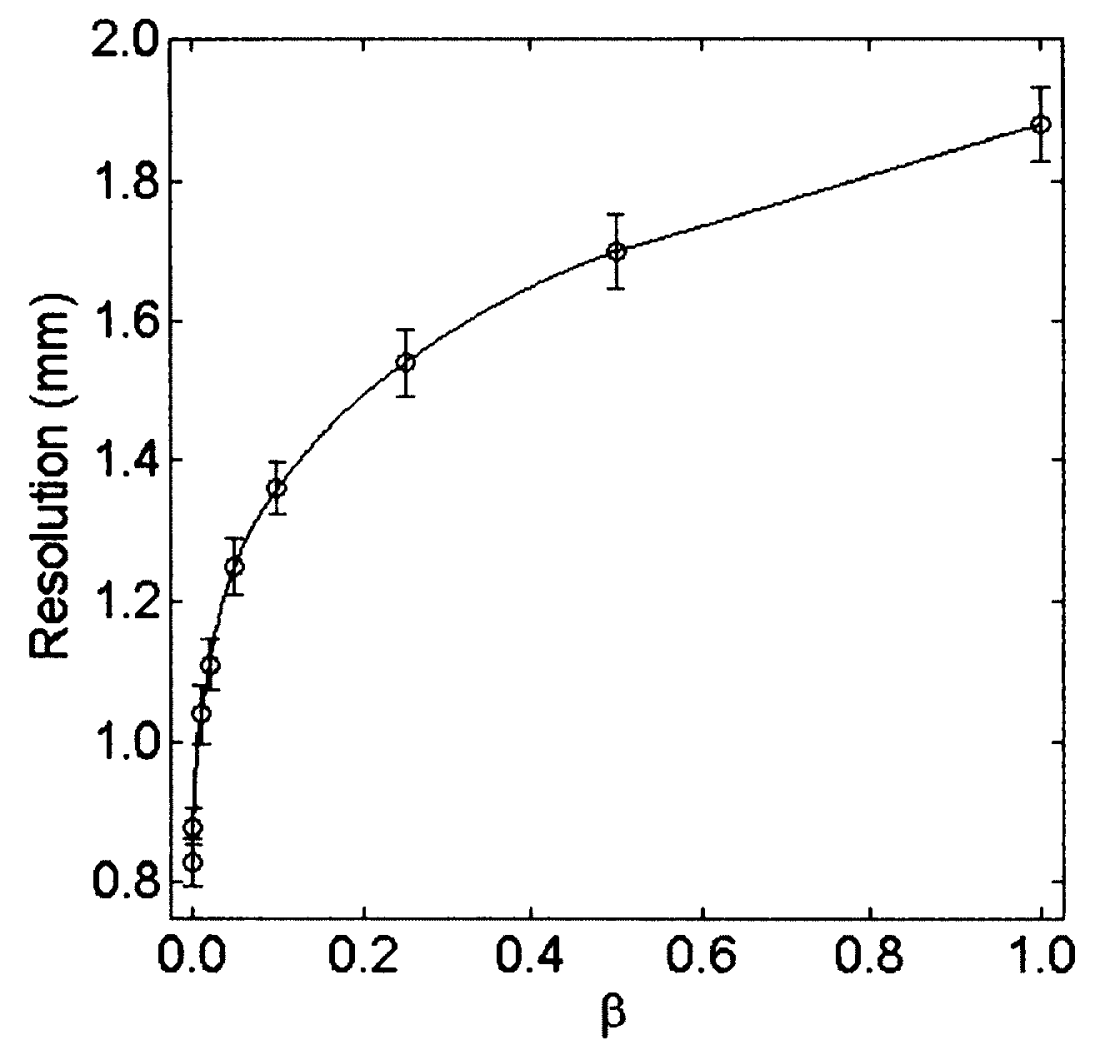

Figure 2.4. Resolution (FWHM) as a function of $\beta$. The results were obtained by fitting a Gaussian function to profiles obtained from images of a line source. 
TABLE 2.1. THE GOODNESS OF FIT $\left(\chi^{2}\right)$ AS A FUNCTION OF $\beta$.

\begin{tabular}{cccccccccc}
\hline$\beta$ & 0 & 0.001 & 0.01 & 0.02 & 0.05 & 0.1 & 0.25 & 0.5 & 1 \\
$\chi^{2}$ & 49.6 & 40.7 & 38.6 & 25.1 & 17.9 & 10.7 & 3.2 & 0.7 & 0.2 \\
\hline
\end{tabular}

A Gaussian PSF is required for the model described in Section 2.2 .1 , thus a $\beta$ value of 0.05 was adopted for simulation and fitting purposes. The average resolution at this $\beta$ was $1.25 \pm 0.04 \mathrm{~mm}$. The results in this section are reported as an average resolution because the tangential resolution was always within $3 \%$ of the radial resolution.

\subsubsection{Noise Properties}

The image of the syringe reconstructed with OSEM3D/MAP reveals that the noise has a correlative component meaning that the process of reconstruction, or the application of the PSF, has the effect of blurring the noise from the raw data (Aston et al. 2002). Although the correlative noise model may not completely describe the noise in the image (Leahy and Qi 2000), the choice of the MAP smoothing prior, $\beta=0.05$, is assumed to generate images that are dominated by correlative noise. Uncorrelated noise, or high frequency noise, is another noise model considered in PET (Aston et al. 2002) which results in additive random noise on the final PET image. Examining the data from the syringe (Figure 2.5(a)), the appropriate model appears to be the correlative noise model because additive random noise is not visible. Thus for simulation, only the correlative noise model is adapted. 

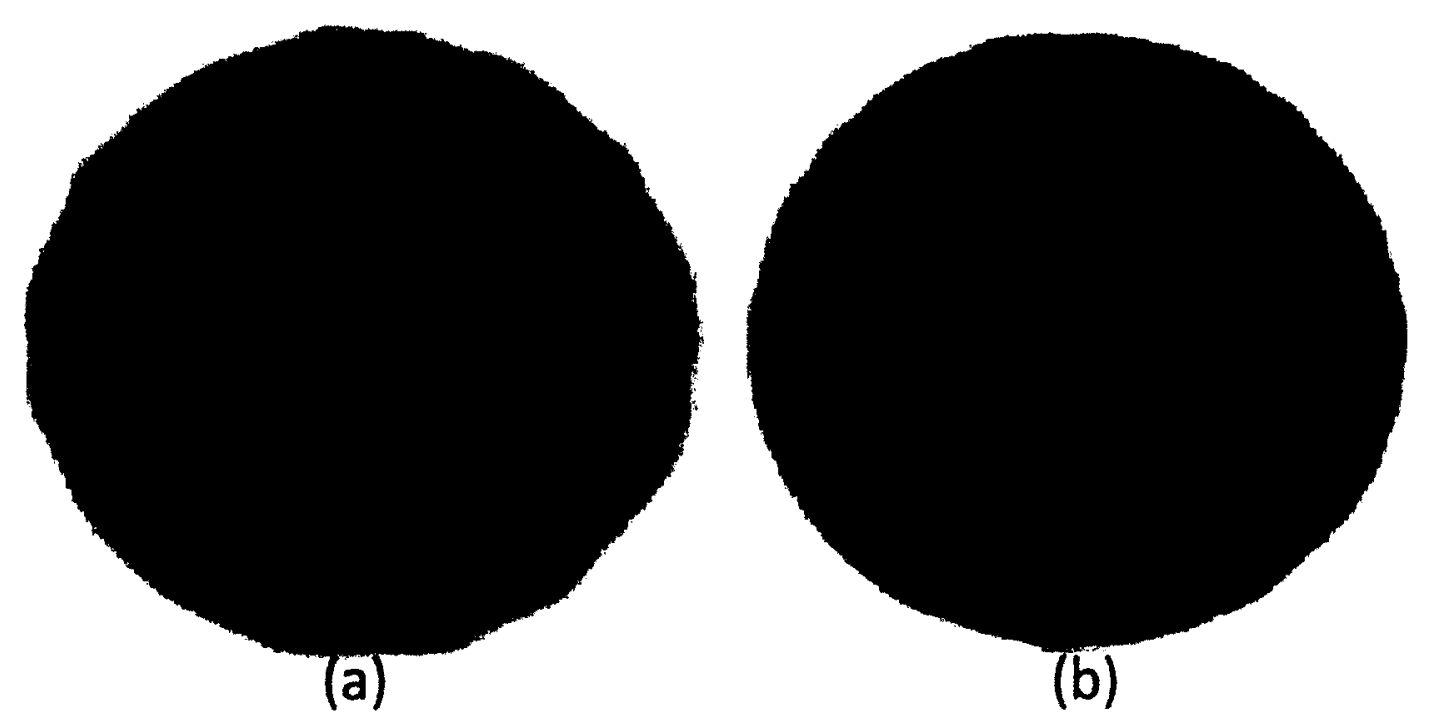

Figure 2.5. Image of a transverse plane of the uniform syringe. (a) The measured PET image and (b) a simulated image with $\mathrm{SNR}=12.5$.

Figure 2.5 illustrates qualitatively how the measured PET image and the simulated image have similar noise properties. Subtle differences in noise textures are noted towards the edge of the phantom. The noise added prior to the PSF operation had a lower SNR of 1.4. The PSF convolution reduces the noise, and thus increases the SNR. The simulated image also had a Gaussian noise distribution even after convolution, supporting the hypothesis that Gaussian noise added prior to applying the PSF serves as an appropriate simulation of the noise properties. 


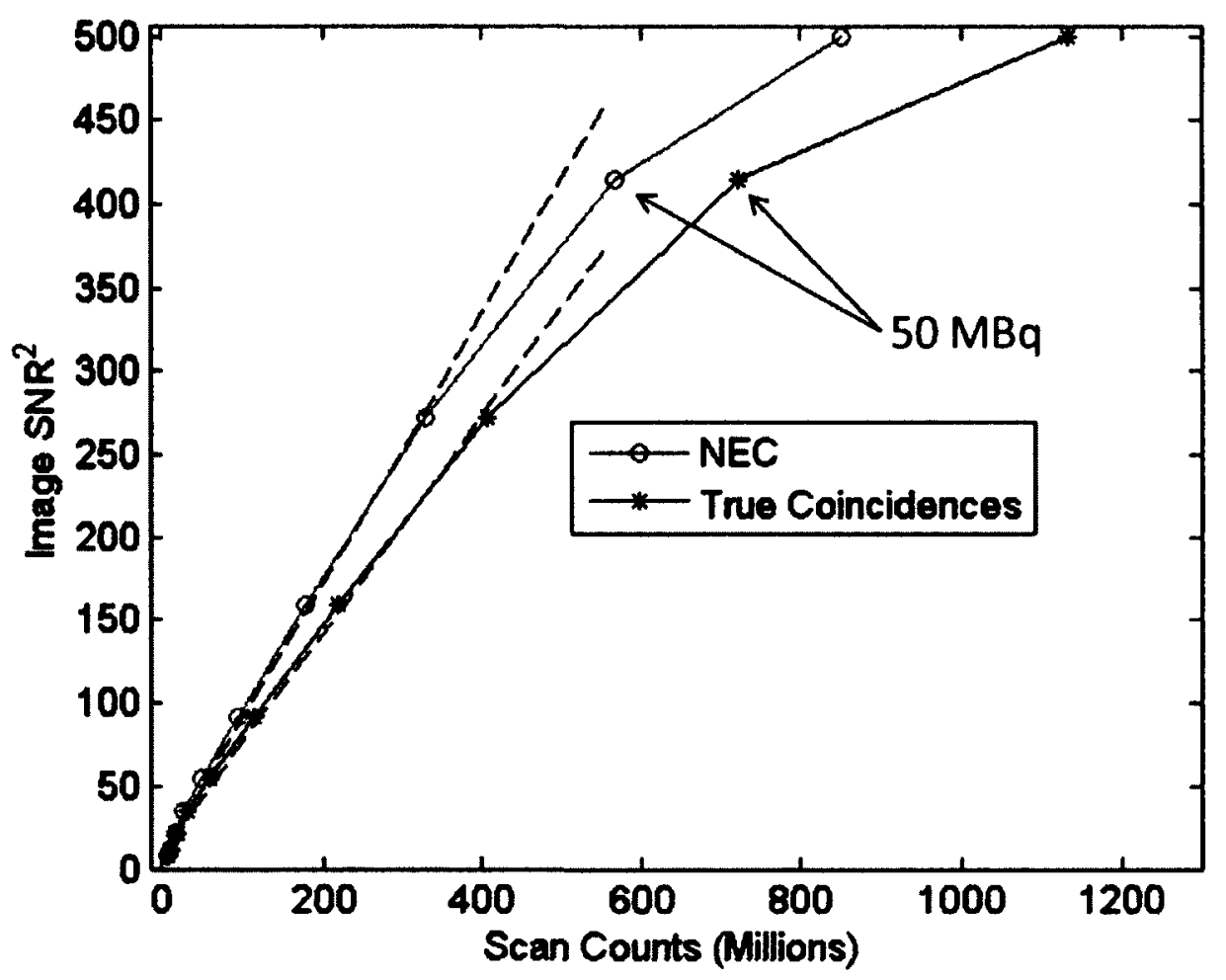

Figure 2.6. Squared SNR as a function of NEC and the true coincidences for a uniform activity distribution with a diameter comparable to a mouse. The linear fits are illustrated by the dashed lines.

The square of the SNR versus the NEC for the syringe study is displayed in Figure 2.6. The quantity is also shown as a function of the true coincidences from the header. The square of the SNR was found to increase linearly $\left(r^{2}>0.99\right)$ with both quantities below 300 million counts. For both lines, deviation from the linear relationship corresponded to an activity of $50 \mathrm{MBq}$. Thus, points corresponding to an activity greater than or equal to $50 \mathrm{MBq}$ were excluded from the linear fit. Mice are typically injected 
with less than $50 \mathrm{MBq}$ of activity, thus for the range of activities used in our mouse studies, a linear relationship is expected to exist. Therefore, noise is added assuming that the $\mathrm{SNR}^{2}$ is proportional to activity in the simulations.

TABLE 2.2. EVALUATION OF SNR IN THE LIVER FOR TWO TIME FRAMES.

\begin{tabular}{cccc}
\hline $\begin{array}{c}\text { Frame Length } \\
\text { (min) }\end{array}$ & $\begin{array}{c}\text { SNR(SNR }{ }^{2} \text { ) in } \\
\text { Liver }\end{array}$ & $\begin{array}{c}\text { Activity Ratio } \\
\text { (liver to } \\
\text { myocardium) }\end{array}$ & $\begin{array}{c}\text { Scaled SNR for } \\
\text { Myocardium }\end{array}$ \\
\hline 1 & $5.87(34.5)$ & 0.5 & 7.6 \\
5 & $8.77(76.9)$ & 0.3 & 15.2 \\
\hline
\end{tabular}

The SNR results from the mouse VOI analysis in the liver are presented in Table 2.2. The SNR increases as expected when a longer time frame is selected. The linear relationship from the data of Figure 2.6 between $\mathrm{SNR}^{2}$ and the true counts can be used to scale the SNR expected in the liver to the SNR in the heart with knowledge of the ratio of activity between the heart and liver. This yielded an expected myocardial SNR of 7.6 for the one-minute frame and an SNR of 15.2 for the five-minute frame. These results provide an estimate of the SNR in the heart that should be simulated for a mouse FDG image. These results assume that frame lengths are between 1 and 5 minutes and that the measured relative uptake is representative of FDG heart imaging. Simulated phantoms were created based on these results, and the background noise was adjusted relative to the myocardial SNR using the relationship of Figure 2.6. An SNR of infinity (no noise) was also simulated for comparison. 


\subsubsection{Partial Volume Correction of the Simulations}

Table 2.3 summarizes the mean and SD for the relative uptake in the organs from $\left[{ }^{18} \mathrm{~F}\right]-\mathrm{FDG}$ mouse PET images. Relative uptake was computed against the $\mathrm{LV}$, thus there was no SD for this organ.

Phantom 1 was simulated in the absence of noise and with a background of 0.22 to assess the algorithm's performance with a concentric uniform wall thickness phantom. Prior to PV-correction the mean recovery, averaged over all gates and angles, was 0.71 . The 1D model fitting led to an improvement with an average $C_{m, I D}$ equal to $0.959 \pm 0.004$ while the $1 \mathrm{D} / 2 \mathrm{D}$ model resulted in an average recovery of $C_{m, 2 D}$ equal to $0.988 \pm 0.001$. The use of the 1D fitting alone improved the recovery substantially, and the 1D/2D model further improved the results $(\mathrm{p}<0.001)$ in comparison to the 1D method, giving a myocardial activity estimate closer to the true activity (i.e. $-1.2 \%$ bias versus $-4.1 \%$ ).

TABLE 2.3. RELATIVE MEAN UPTAKE VALUES \pm SD FROM MICE INJECTED WITH $\left[{ }^{18} \mathrm{~F}\right]$-FDG.

\begin{tabular}{ccccccc}
\hline $\begin{array}{c}\text { Time } \\
\text { frame }\end{array}$ & $\begin{array}{c}\text { LV } \\
\text { Myocardium }\end{array}$ & $\begin{array}{c}\text { LV } \\
\text { Blood }\end{array}$ & $\begin{array}{c}\text { Back- } \\
\text { ground }\end{array}$ & Liver & $\begin{array}{c}\text { RV } \\
\text { Myocardium }\end{array}$ & $\begin{array}{c}\text { RV } \\
\text { Blood }\end{array}$ \\
\hline Early (5- & 1 & 0.04 & 0.14 & 0.27 & 0.34 & 0.21 \\
10 mins) & 1 & \pm 0.04 & \pm 0.05 & \pm 0.07 & \pm 0.10 & \pm 0.05 \\
& & 0 & 0.06 & 0.04 & 0.21 & 0.10 \\
Late (55- & 1 & \pm 0 & \pm 0.02 & \pm 0.01 & \pm 0.06 & \pm 0.04 \\
60 mins) & 1 & & & & & \\
\hline
\end{tabular}


Phantoms 2 and 3 were studied to examine how $C_{m, I D}(\theta)$ and $C_{m, 2 D}(\theta)$ vary with the average myocardial thickness (for a given angle). In both cases a background of 0.22 was simulated. The results are summarized in Figure 2.7. The average $C_{m, 2 D}$ computed over all 36 angles, was $0.993 \pm 0.026$ for phantom 2 and $0.953 \pm 0.074$ for phantom 3 .

Again, there was a substantial improvement in the estimated myocardial activity when performing the 1D fitting. The 1D/2D model further improved the accuracy for almost all angles. At larger wall thicknesses, the algorithm tended to overestimate the true activity slightly in comparison to the 1D fitting alone. Below $0.4 \mathrm{~mm}$, there was more error in comparison to the larger wall thicknesses. There was a significant improvement in the average recovery between the $1 \mathrm{D}$ and combined $1 \mathrm{D} / 2 \mathrm{D}$ corrections $(p<0.001)$. For phantom 2 the bias was improved from $-4.2 \%$ to $0.0 \%$, while for phantom 3 the bias was improved from $-9.0 \%$ to $-4.7 \%$.

For phantoms 1-3 the error on $I_{m y o}$ was assessed with background activity equal to 0.22 . Figure 2.8 illustrates the difference between $I_{m y o}$ and the true myocardium of the phantoms for end-diastole and end-systole. The mean error on wall thickness estimates along with associated maximum and minimum values from the $1 \mathrm{D}$ model are summarized in Table 2.4 .

The 1D algorithm underestimated the true wall thickness as shown in Table 2.4. The largest walls in phantoms 2 and 3, at end-systole, are subject to the largest errors and these errors result in some overcorrection as shown in Figure 2.7. 

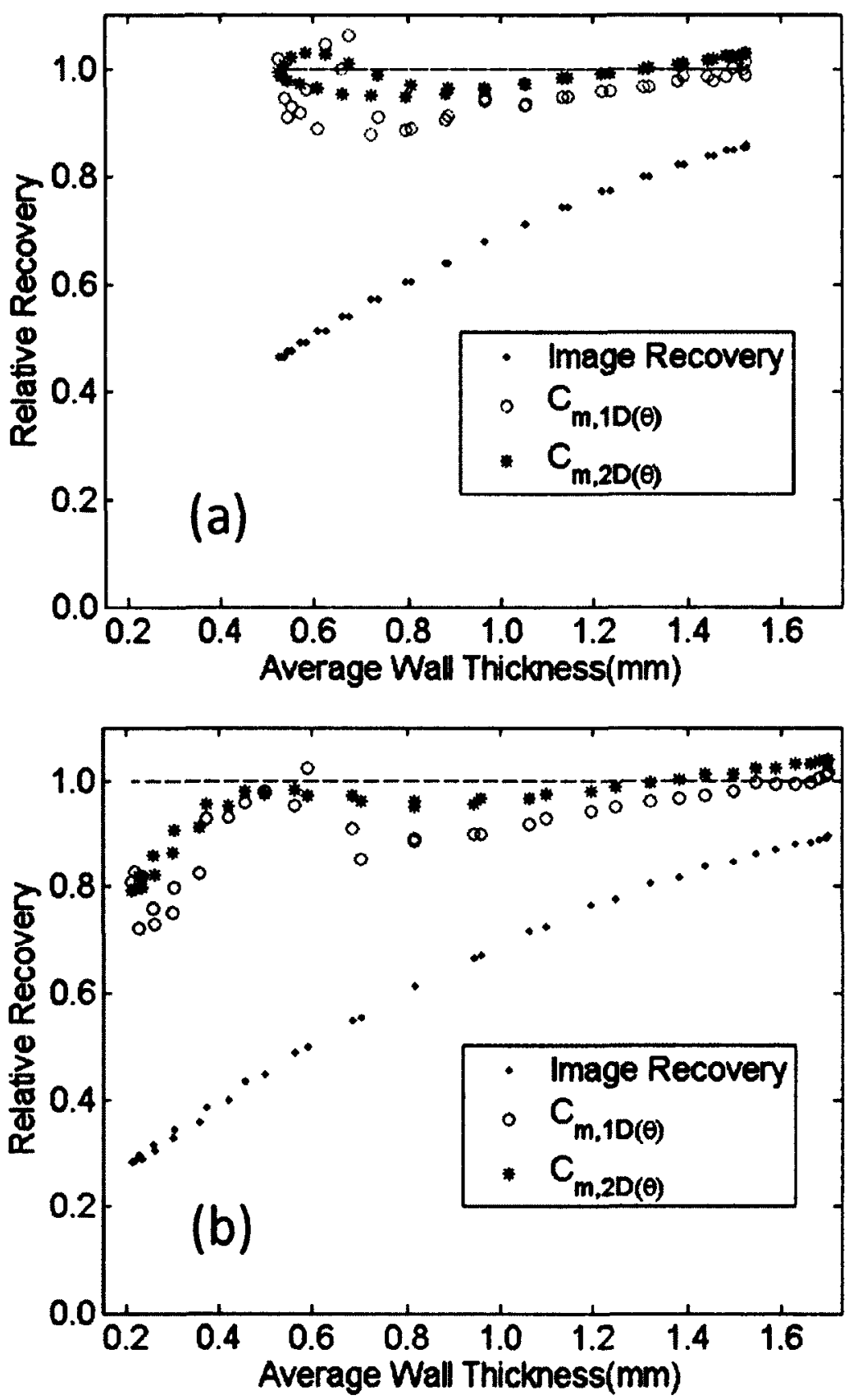

Figure 2.7. Recovery of activity as a function of the average wall thickness. Each point represents one angle through the myocardium. The dashed line represents the true activity. The dots represent the maximum recovery in the images without partial volume correction. The open circles give the myocardium estimation with the 1D model $\left(C_{m, 1 D}(\theta)\right)$, while the stars represent the results with the 1D/2D model $\left(C_{m, 2 D}(\theta)\right)$. (a) The results for phantom 2 and (b) the results for phantom 3. Note that the results from phantom 3 have a larger range in the average wall thickness simulated. 
TABle 2.4. ERrors on WALl Thickness Estimates from the 1D MODEl.

\begin{tabular}{cccc}
\hline Phantom & $\begin{array}{c}\text { Mean error on } \\
\text { wall thickness } \\
(\mathrm{mm})\end{array}$ & $\begin{array}{c}\text { Minimum error } \\
\text { on wall } \\
\text { thickness }(\mathrm{mm})\end{array}$ & $\begin{array}{c}\text { Maximum error } \\
\text { on wall } \\
\text { thickness }(\mathrm{mm})\end{array}$ \\
\hline 1 & -0.03 & -0.06 & 0.01 \\
2 & -0.06 & -0.25 & 0.04 \\
3 & -0.05 & -0.31 & 0.05 \\
\hline
\end{tabular}

\section{Phantom 1}

\section{End Diastole End Systole}

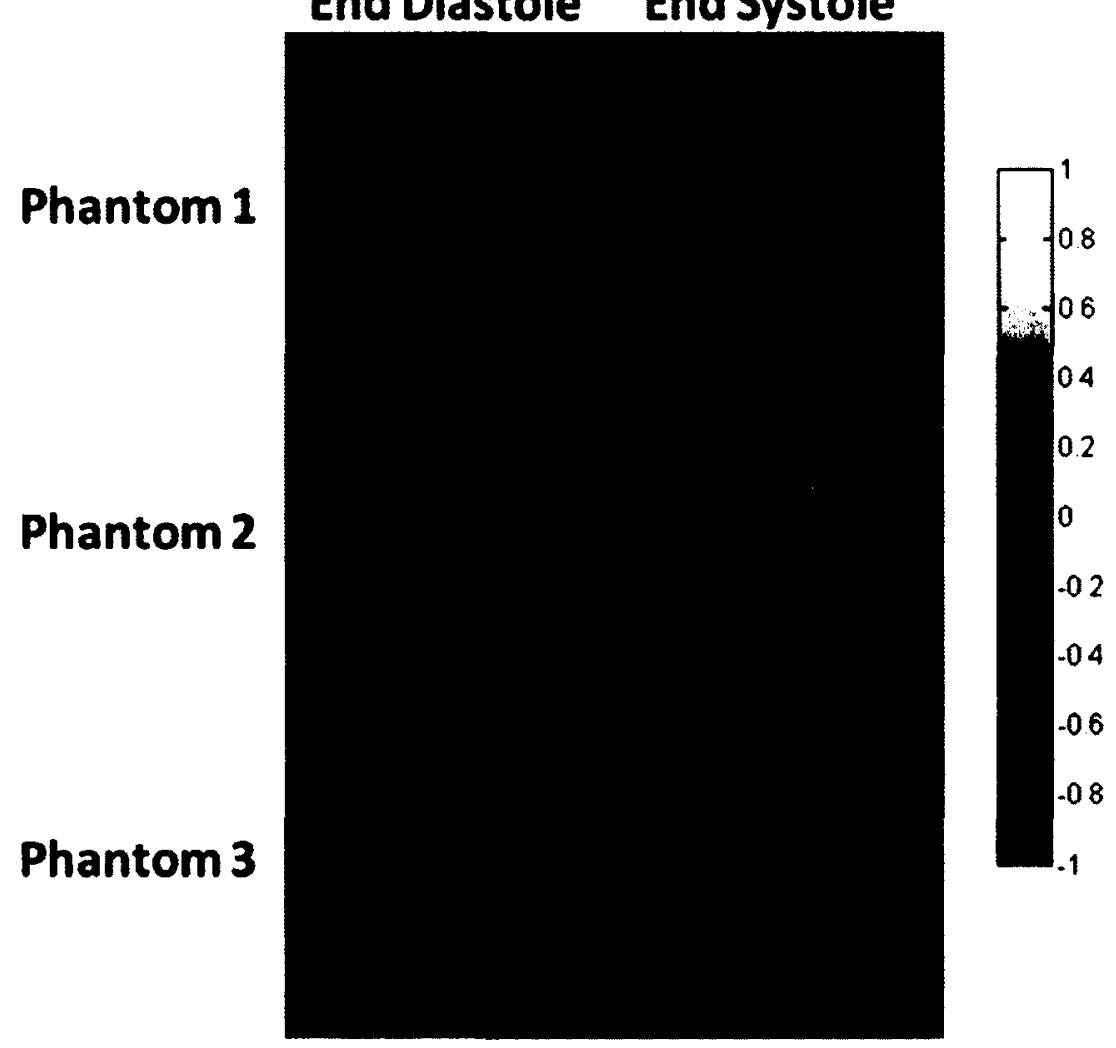

Figure 2.8. Difference between the estimated model $I_{\text {myo }}$ and the true phantom myocardium in end-diastole and end-systole for phantoms 1-3.

The simulated MOBY phantoms with the two different background distributions (Table 2.3) were used to examine how noise affects the PV correction algorithm. The end-diastolic images simulated from the early time frame of Table 2.3 are shown in Figure 2.9. The SNRs that were simulated were infinity (no noise), 15.2 and 7.6. 


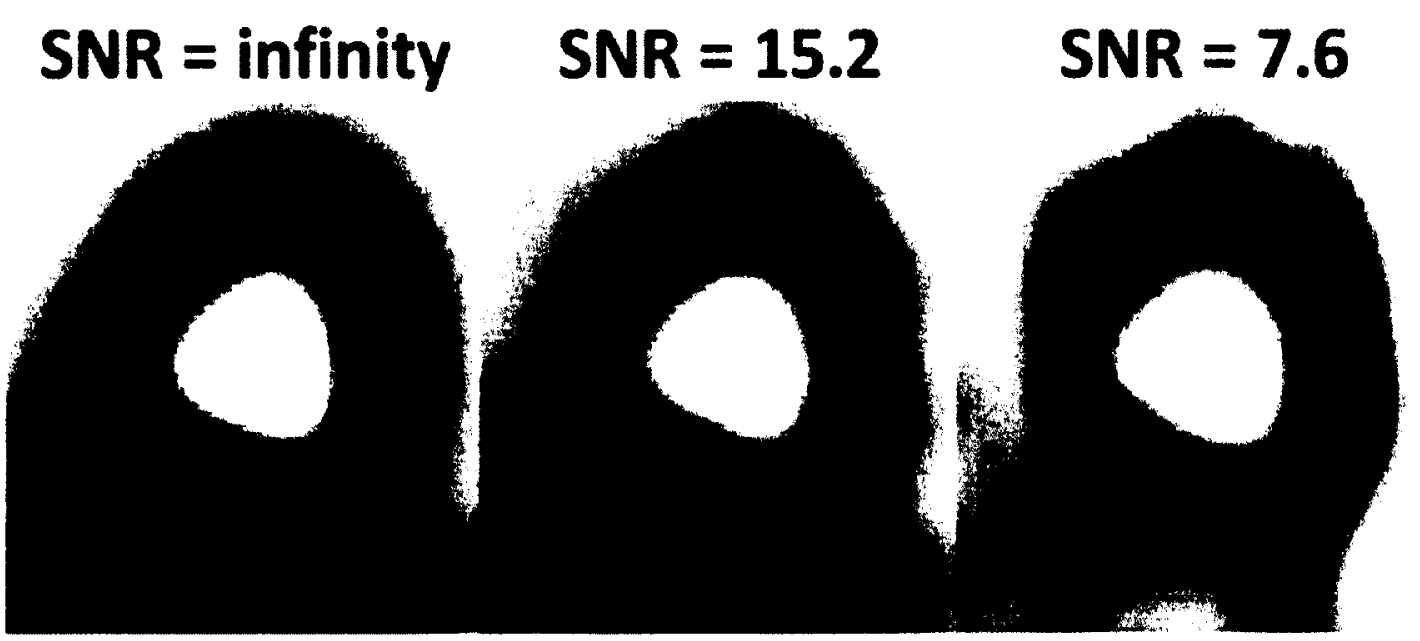

Figure 2.9. End-diastolic images of the simulated MOBY phantom at five minutes post-injection for different SNRs.

There is a noticeable deterioration in image quality with increasing noise. $C_{m, 2 D} \pm$ SD is plotted for both MOBY phantoms in Figure 2.10. For the SNRs of 7.6 and 15.2, the PET image was simulated ten and five times respectively. $C_{m, 2 D}$ and the SD were computed for each trial and averaged over all trials to obtain one estimate for each SNR. In the case of no noise, only one trial was performed. The figure also displays the recovery before correction and the true activity for comparison.

In both cases, the average recovery is substantially improved by PV correction. Interestingly, the average recovery value appears to be closer to the truth under noisy conditions. There is a slight bias in the results for both phantoms with SNRs of infinity and 15.2 . The bias ranged from $3-5 \%(p<0.001)$. The underestimation of activity with noiseless conditions is attributed to the ID model's inability to perfectly estimate the parameters. As expected, in both cases the standard deviation on $C_{m .2 D}$ was highest when 
the SNR value was lowest. In the case of Figure 2.10(a) with an SNR of 15.2, the results were actually superior to the case of no noise in terms of both $C_{m, 2 D}$ and standard deviation. In the case of Figure 2.10(b) the average recovery increases in accuracy with decreasing SNR but with a loss of precision. These results demonstrate that the algorithm can perform well even in the presence of realistic noise levels.

The LV blood activity was found to be small in the animal studies but the background had a wide range of values due to the surrounding structures. The relative blood activity was 0.01 . Relative backgrounds of $0.02,0.22$ and 0.41 were examined under SNRs of infinity and 15.2 in phantoms 1-3 to study the effect of background on the model. The blood $\left(C_{b}\right)$ estimated from the $1 \mathrm{D}$ model overestimated the true blood activity by $1-7 \%$ relative to the myocardium. The background $\left(C_{g}\right)$ estimated from the $1 \mathrm{D}$ model was within $1-3 \%$ relative to the myocardium. The mean $\pm \mathrm{SD}$ recovered myocardial activity for the $1 \mathrm{D} / 2 \mathrm{D}$ model was plotted as a function of the relative background in Figure 2.11 for phantoms $1-3$. The top graphs represent the results in the presence of noise, while the bottom graphs represent the absence of noise.

The SD of phantom 1 was not displayed on the graphs because the data fell within the circles on the graph. For phantoms 1 and 2 the corrected activity was close to unity with and without noise and across all relative background activities. For phantom 2 , the correction also substantially decreased the variability which originated from the different wall thicknesses encountered. For phantom 3, the corrected activity was within $5 \%$ for relative background levels of 0.02 and 0.22 with and without noise. When the background was increased to 0.41 the corrected activity was underestimated by $8 \%$ when no noise was present and overestimated by $10 \%$ when noise was present. The precision 
was also reduced in phantom 3 when the background was 0.41 in comparison to all of the other data points of Figure 2.11.
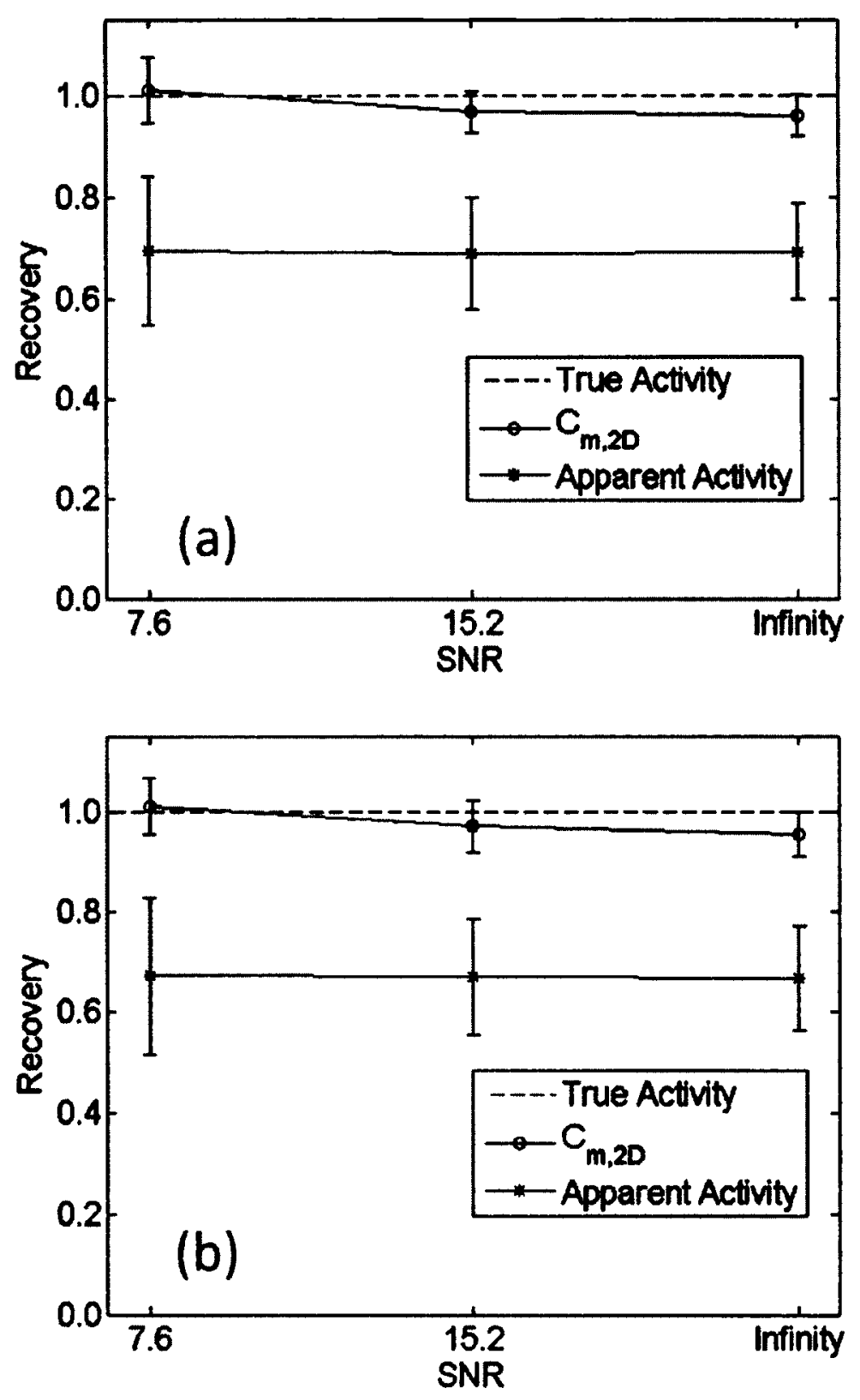

Figure 2.10. The effect of noise on the partial volume recovery algorithm in the MOBY phantoms. (a) Background values are derived from the early time frame of Table 2.3 and (b) background values are derived from the late time frame of Table 2.3. The recovery prior to correction is denoted with the asterisks while the corrected values are denoted by the open circles. 

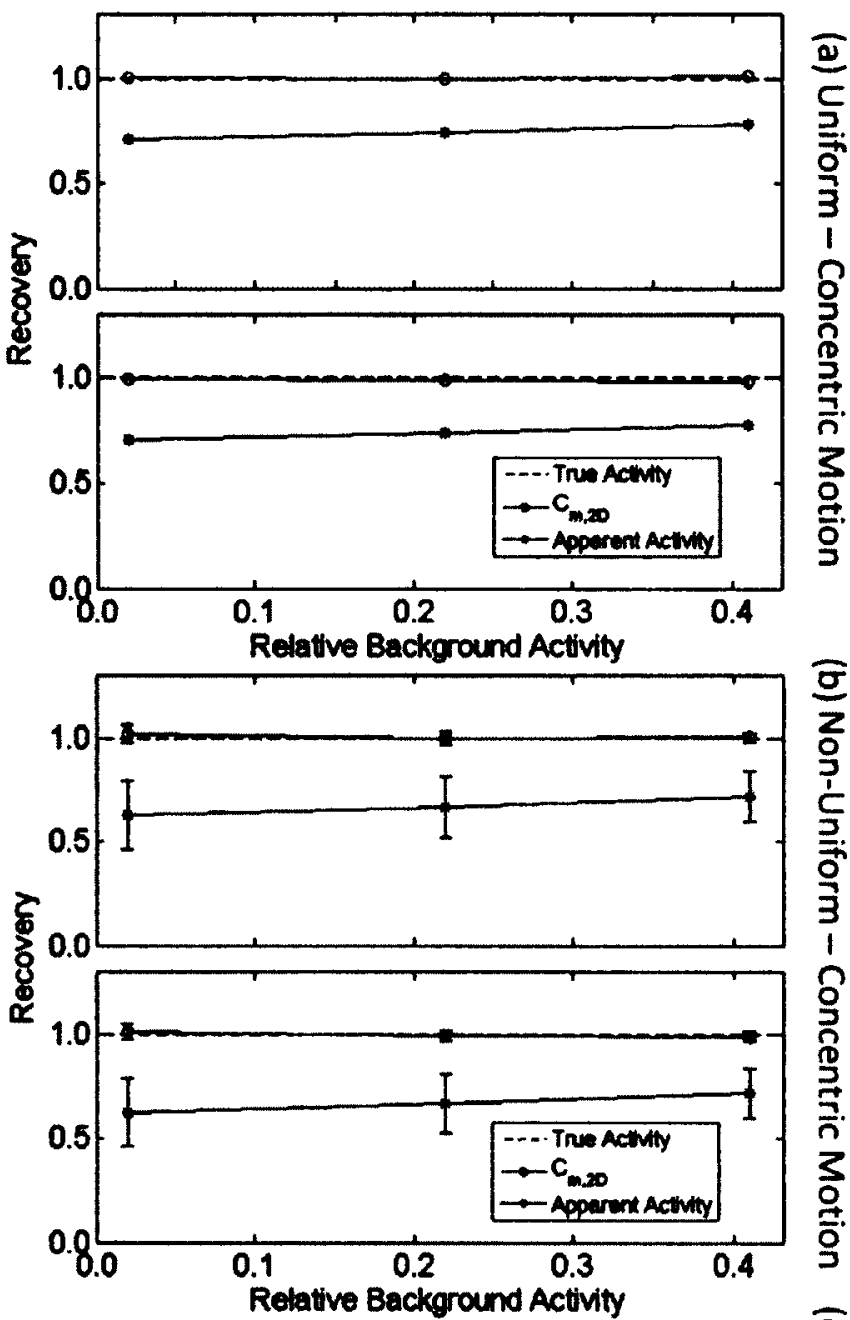

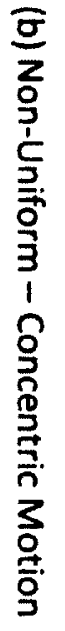

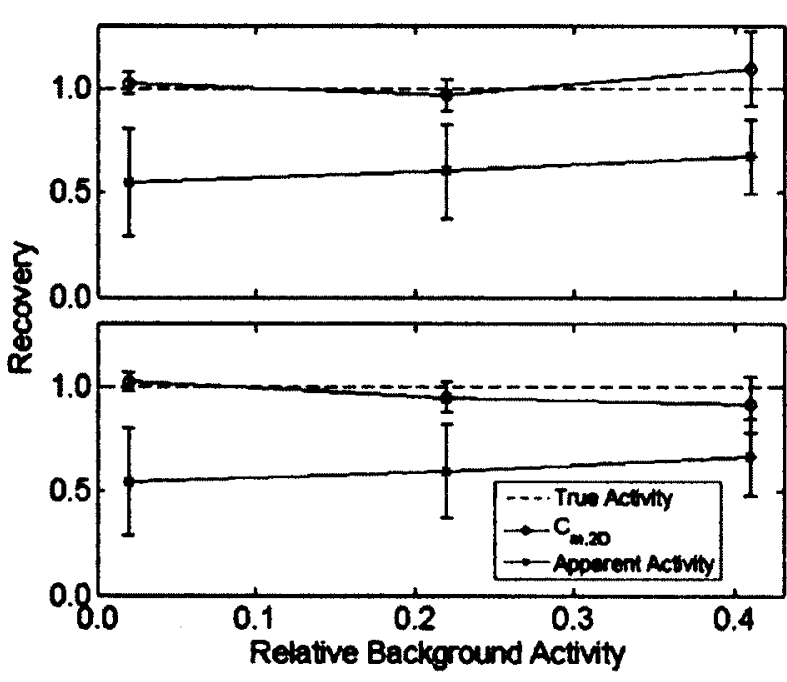

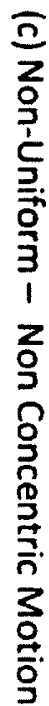

Figure 2.11. Recovery versus relative background activities in (a) phantom 1, (b) phantom 2 and (c) phantom 3. The bottom graphs have an SNR of infinity while the top graphs have an SNR of 15.2. 


\subsection{Discussion}

The 1D fitting algorithm alone was found to substantially improve the recovered activity in the LV myocardium of a simulated mouse heart geometry with contractile motion. Using the LV myocardial wall, blood and background information from the ID fitting algorithm, the results were improved further by subtracting the simulated background from a PET image and then deriving RCs by convolving the 2D myocardial estimate with the PSF. This 1D/2D model performed well in a variety of situations, but is subject to limitations due to noise, very thin walls and high background activity.

The correction of the uniform phantom demonstrates that the algorithm can yield myocardial activity estimates close to the true activity distribution. The small standard deviation demonstrates that fitting along various angles in a uniform LV is precise, ensuring that the correction can reproduce the same results independent of the sampling angle intersecting the myocardium.

With the 1D/2D model, it was found that the algorithm was less accurate when very small wall thicknesses $(<0.4 \mathrm{~mm})$ were encountered as indicated in Figure 2.7. Phantom 2 had a relatively constant corrected recovery close to the true value, across the range of wall thicknesses $(0.5-1.5 \mathrm{~mm})$ leading to a small variability in the myocardial activity estimates. Phantom 3 had much more variability as expected in the very thin walls simulating infarcted tissue. The problem with fitting small wall thicknesses is that the borders of the wall were more difficult to define due to the tissue fraction effect. Figure 2.3 demonstrated that for phantom 3 there was not full recovery in the thinnest part of the wall, even prior to convolving the phantom image with the PSF. After convolution, the wall activity spilled into the background, but because the background 
had a comparable uptake to the simulated wall, the algorithm had more difficulty discriminating between the wall and background activities. For the case of a $0.2 \mathrm{~mm}$ wall thickness, the algorithm should ideally produce a wall thickness estimate of exactly 1 pixel. However, a wall thickness estimate greater than 1 pixel was found, resulting in an underestimated activity because the RC was overestimated. Furthermore, phantom 3 had little motion for the smallest wall thicknesses. Thus, the benefit of wall thickening was not exploited to increase the accuracy of the correction as in phantom 2. The large underestimation of activity in the small walls was not observed in phantom 2, mainly because there was adequate recovery in the smallest wall $(>0.5 \mathrm{~mm})$ and there was normal wall thickening. Another interesting feature was noted in the results for phantom 3. In the small wall thicknesses there is a rapid rise in recovery, peaking at an average wall thickness of $0.5 \mathrm{~mm}$, because of the contrast improvement from the diminished tissue fraction effect. The peak may signify that either the chosen initial conditions, or the fitting intervals are optimized under these wall thicknesses for this particular phantom. The slight underestimation of recovery between $0.8 \mathrm{~mm}$ and $1 \mathrm{~mm}$ is common to both phantoms and is due to overestimating the myocardial wall thicknesses slightly using the 1D model. Narrowing the fitting interval in the 1D model to exclude more blood and background activity could help improve the accuracy but less data would be available for fitting. In phantoms 2 and 3, however, the algorithm appeared to perform well in the range of average wall thicknesses between 0.6 and $1.4 \mathrm{~mm}$ which are typical of the normal LV wall thickness in mice. For wall thicknesses greater than $1.4 \mathrm{~mm}$ the $1 \mathrm{D} / 2 \mathrm{D}$ model slightly overestimated the recovery because the fitting interval was not wide enough to accurately describe the full wall thickness. This overestimation could be 
corrected, however, wall thicknesses greater than $1.4 \mathrm{~mm}$ are uncommon.

The simulated SNR was observed to have an impact on the image quality as expected (Figure 2.9) and the ability of the algorithm to perform an accurate PV correction (Figure 2.10). Some underestimation of activity was expected before correction, because the wall thicknesses encountered in this phantom were on the order of $1 \mathrm{~mm}$. When noise was present, the 1D fitting detects a thinner myocardial wall contour which translates into a larger correction factor. This increased the accuracy of the correction even though variability on the estimate was increased.

Figure 2.11 demonstrates the $1 \mathrm{D} / 2 \mathrm{D}$ model performance in a variety of background activities and noise typical to FDG heart imaging. When noise was present the variability in the estimated myocardial activity was larger, although the average myocardial activity was still observed to be close to unity with and without noise. In the case of phantom 3, there was more variability in the corrected values in comparison to the other two phantoms, with the most variability being recorded for highest background. This increased variability can be attributed partially to the tissue fraction effect in very thin walls, as discussed previously. When noise was added the borders were even more difficult to detect. This is clear from Figure $2.11(\mathrm{c})$ as the overall variability in the data is largest for this case. In general, the contractile function (wall thickening) of the heart can help average out these effects, however, there was no contraction in the thinnest wall of this phantom. Thus, averaging the estimated myocardial activity values throughout the cardiac cycle did not aid in improving the values. Therefore, this algorithm is most sensitive to correcting PV effects in infarcted tissue, especially in high background and under noisy conditions. Infarcted regions require the largest correction factors and are 
thus most sensitive to errors. An advantage to using the 1D/2D model is that over/under estimated wall thicknesses are interpolated onto a $2 \mathrm{D}$ grid. Thus, if the neighboring thicknesses are accurate, the PSF smoothing will help to reduce these over/under estimates locally. Although the algorithm is most sensitive to infarcted areas in the heart, it still provides an estimate that is much closer to the true activity; e.g. an improvement from $30 \%$ recovery to $80 \%$ recovery in $0.2 \mathrm{~mm}$ walls.

The accuracy of the PSF is a vital component of the $1 \mathrm{D}$ and $1 \mathrm{D} / 2 \mathrm{D}$ models. The selection of $a \beta$ of 0.05 ensured that the PSF had a Gaussian shape. However, it was also assumed that the width of the PSF was well characterized. A sensitivity analysis was performed with phantom 1 such that the FWHM of the PSF was over/underestimated. Phantom 1 was simulated with no noise, a background of 0.22 and a PSF with a FWHM of $1.25 \mathrm{~mm}$. The 1D/2D model was implemented with a FWHM of 1.2 (and 1.3) mm. The model was found to underestimate or overestimate the myocardial activity by approximately $5 \%$ when the smaller or larger FWHM values were used respectively. This demonstrates the need for accurately measuring the PSF prior to performing corrections. When considering studies performed in a PET scanner, the PSF should be measured in soft tissue ideally with count rates that are typical of FDG mouse heart imaging. This ensures the PSF is measured under realistic imaging conditions.

This chapter focused on a 2D correction model based on 1D model fitting. The 1D model was fit independently in each angle, neglecting local curvature but producing a robust fit. A fully 2D model could be beneficial but assumptions on the geometrical shape of the myocardial activity distribution would be needed. Describing a higher order spatial dimension requires more model parameters to take into consideration local 
curvature of the LV myocardium and this may be less reliable than the simpler 1D methodology.

This work assumed a spatially invariant resolution throughout the field of view, however, this is not a requirement of the model. The only requirement for the 1D model presented in Figure 2.1 is that the PSF be spatially invariant along the 1D profile. If the PSF was known, the model could be adjusted to incorporate spatial variance, and the smoothing operations of equations 2.2 and 2.3 could also be modified accordingly.

Anatomic images are not required for the presented algorithm, however, highresolution anatomic images could be beneficial in delineating the myocardium. With a uniform activity distribution assumed radially across the myocardial thickness, as examined in this chapter, the mean error on wall thickness was on the order of the pixel size. Coregistration and segmentation of an anatomic image would likely lead to similar errors. Thus, in the cases studied, anatomic imaging may or may not offer substantial improvement in accuracy. The methodology was most limited under high background and noise conditions in the non-uniform non-concentric phantom with the $0.2 \mathrm{~mm}$ wall. Under these conditions, an anatomic image may aid in accurate delineation of the thinnest wall.

The activity distribution across the LV myocardium thickness may be nonuniform although the presented model assumed that the uptake was uniform in the radial direction. PET measures an average transmural activity due to intrinsic resolution limitations. Subtle variations in myocardial activity across a healthy myocardium cannot be distinguished because the PET image represents an average activity over LV wall thickness. However, in the case of a subendocardial (partial-thickness) infarct, there 
could be distinct regions across an LV myocardial profile with different uptake and the 1D algorithm would have difficulty resolving the myocardial walls. In these cases an anatomic image may also be useful in aiding wall delineation. Furthermore, the method was tested with phantoms that had homogeneous activity in the myocardium. The model only assumes that there is homogeneous activity in the radial direction and makes no assumption on the activity distribution in the circumferential direction.

In addition to scanner resolution, contractile and respiratory motion limit the ability to quantify LV myocardial activity. In this study, contractile motion was eliminated by ECG gating. In contrast, respiratory motion was not considered, as this effect is more subtle than cardiac motion and affects less than $25 \%$ of the cycle (Yang et al. 2005). In future studies, dual-gating could be used and the effect of respiratory motion could be minimized simply by excluding the fraction of the data where the motion is most significant.

The noise simulations performed were based on the noise properties from a single PET measurement with the $30 \mathrm{~mL}$ syringe filled with an $\left[{ }^{13} \mathrm{~N}\right]$-solution. To be more comparable to a Monte Carlo simulation, ensemble noise properties could be derived by obtaining multiple realizations of the same source and deriving the SNR across the realizations. This could help produce simulated images that are more representative of the true noise distribution. However, the simulation method described in Section 2.2.4 is computationally efficient and eliminates the need for multiple data acquisitions.

The goal of the combined 1D/2D model is to provide an accurate and automated approach for PV correction for mouse heart imaging. The methodology could also be applied to other species as well. While the developed model was constructed for short- 
axis 2D images, it may be extended for use on 3D LV myocardial images. The model makes an assumption that there are minimal PV losses along the dimension perpendicular to the heart wall. This is true towards the base of the heart, but not at the apex where the LV myocardium begins to curve in the $3^{\text {rd }}$ dimension. To apply the $2 \mathrm{D}$ model to these images, 2D images must be formed from the myocardium such that there are minimal PV losses along the axis perpendicular to the plane chosen. These images can be obtained by sampling the apex radially outward from the center of mass of the myocardium (Lortie $e t$ al. 2007). With a proper 3D sampling process, it could be possible to use the algorithm to fully correct 3D mouse images of the entire LV myocardium.

\subsection{Conclusion}

The combined $1 \mathrm{D} / 2 \mathrm{D}$ model developed in this study has been demonstrated to reduce PV effects in simulated LV myocardial mouse heart imaging. The model provides accurate estimates of the true myocardial activity in a variety of situations. Wall thickness, wall motion, noise and background activity were all observed to affect the performance of the algorithm, although under typical conditions the algorithm was accurate to within $5 \%$, on average. One limitation of the algorithm was that very thin infarcted tissue was difficult to correct, because of the tissue fraction effect. Although this limits the correction accuracy, the 1D/2D model still offers a substantial improvement of recovered activity in infarcted regions. The model also has benefits in that it does not require coregistered anatomic images, it can be easily automated and it takes into consideration contractile motion by using ECG-gated images. The 1D/2D 
model thus offers a practical solution to the PV problem and should present an improvement to quantitative $3 \mathrm{D}$ heart imaging. 


\section{Chapter 3. Experimental Validation of the 1D/2D Partial Volume Correction Method and Extension to 1D/3D}

Preliminary results for the work presented in this chapter were published in abstract form (Dumouchel and deKemp 2010a).

(C) 2010 IEEE. Reprinted with permission, from Tyler Dumouchel and Robert A. deKemp, Recovery of Partial Volume Losses in Cardiac Mouse PET Imaging using a Combined 1D/2D and a Combined 1D/3D Model, 2010 IEEE Nuclear Science Symposium Conference Record, October 30, 2010 - November 6, 2010 (Dumouchel and deKemp 2010b). This paper has been reformatted, expanded upon, the text has been edited and figures have been converted to black and white to conform to the thesis format.

\subsection{Introduction}

In this chapter, the combined 1D/2D algorithm of Chapter 2 is validated using a physical 2D mouse heart phantom designed to emulate a $2 \mathrm{D}$ short-axis gated mouse heart. The algorithm is also tested in vivo on basal slices of $\left[{ }^{18} \mathrm{~F}\right]-\mathrm{FDG}$ gated cardiac mouse PET images. 
As discussed in Section 1.11, PET is a 3D imaging modality and PV effects are expected along all spatial dimensions. In the base region of the LV, where the LV myocardium is sampled in cylindrical coordinates (Figure 1.7), the 1D/2D model was able to reduce PV losses (Chapter 2). However, in the apex region of the LV myocardium where the LV myocardium geometry is sampled in spherical coordinates, it is predicted that the $1 \mathrm{D} / 2 \mathrm{D}$ model is insufficient to fully describe PV losses. Following a similar methodology to the $1 \mathrm{D} / 2 \mathrm{D}$ algorithm, a $1 \mathrm{D} / 3 \mathrm{D}$ algorithm is proposed and the algorithm is tested using 3D digital simulations.

\subsection{Materials and Methods}

\subsubsection{The ID/2D Partial Volume Correction Algorithm}

The combined 1D/2D PV correction algorithm used is explained in Section 2.2.1.

\subsubsection{The 2D Gated Mouse Heart Phantom}

The 1D/2D implementation of the algorithm was tested on a physical phantom that was manufactured such that resolution losses only occur in 2D, along the transverse dimensions $(x, y)$. The phantom had variable wall thickness, and cardiac motion was approximated by dividing the phantom into eight transverse planes where each plane represents a particular ECG-gated cardiac phase. Plane thicknesses were made large enough such that axial resolution losses were minimal to ensure that each plane in the reconstructed image could be considered independently in 2D. The phantom had a wall thickness of $0.4-1.4 \mathrm{~mm}$ in end-diastole and $0.7-1.7 \mathrm{~mm}$ in end-systole. The phantom was designed to have an ejection fraction of $64 \%$, which is typical of a healthy mouse 
(Steggar et al. 2009). The heart was designed to emulate uniform contraction, similar to a real beating heart (Segars et al. 2004). A Fourier series was used to describe the endocardial wall motion as a function of gate while conservation of wall area was used to define the epicardial wall contour contraction (Section 2.2.5). There was no wall motion between gates. The phantom was constructed from clear cast acrylic that has a linear attenuation coefficient of $0.111 \mathrm{~cm}^{-1}$ at $511 \mathrm{keV}$ (Berger et al. 1999). This attenuation coefficient is larger than that of water $\left(0.096 \mathrm{~cm}^{-1}\right.$, Berger et al. 1999) which is comparable to soft tissue. Thus it was possible that this phantom was not completely representative of small animal tissue. However, both attenuation coefficients are dominated by Compton scattering (Berger et al. 1999) and at $511 \mathrm{keV}$ small-angle forward scattering is favoured (Cherry et al. 2003). Thus, although there was a $14 \%$ difference between attenuation coefficients, it was believed that using tissue equivalent plastic would not significantly alter the results. The $2 \mathrm{D}$ mouse heart phantom design is illustrated in Figure 3.1. The sagittal axis view illustrates the eight different planes that were constructed (dashed lines), where each plane corresponds to a specific ECG-gated cardiac phase. A transverse view is also shown for the plane representing end-diastole with wall thicknesses ranging from 0.4 to $1.4 \mathrm{~mm}$. The locations of each corresponding view are illustrated by the black dashed line on each respective image. The white region in the phantom represents the fillable chamber while the gray regions represent the clear cast acrylic. The different shades of phantom material are for illustrative purposes, showing the separate machined components. 


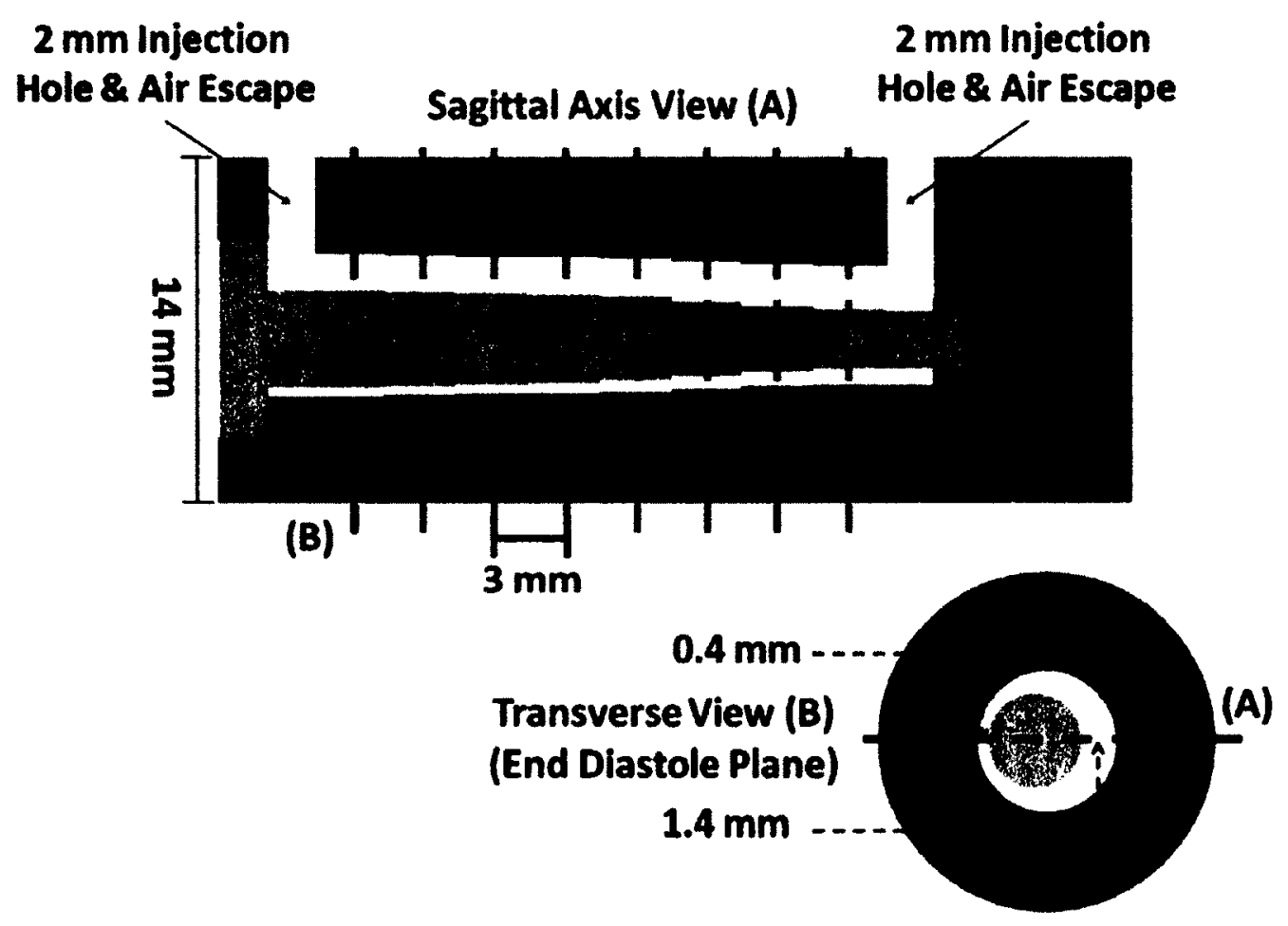

Figure 3.1. The 2D variable wall thickness mouse heart phantom used to test the 1D/2D PV correction algorithm.

The phantom was filled with $19 \mathrm{MBq} / \mathrm{mL}$ of $\left[{ }^{18} \mathrm{~F}\right]-\mathrm{FDG}$ and scanned with the Inveon for 150,600 and $1800 \mathrm{~s}$ with the phantom positioned close to the center of the field of view. Data were binned to 3D sinograms using a ring difference of 79 and a span of 3. Images were reconstructed using the OSEM3D/MAP algorithm with 2 OSEM3D iterations, $18 \mathrm{MAP}$ iterations and $\beta$ set equal to 1, to ensure a Gaussian PSF response and to increase the SNR, with uniform resolution and fastMAPTM enabled. The images were reconstructed onto a $256 \times 256 \times 159$ matrix with $0.2 \times 0.2 \times 0.8 \mathrm{~mm}^{3}$ pixels. The activity concentration was uniform throughout the entire phantom. Therefore, the 2D PV correction algorithm described in Section 2.2.1 was performed with the image data. The central transverse plane from each section, as denoted by the vertical lines in Figure 3.1 
was extracted. The PV correction was applied with a $\Delta \theta$ of $10^{\circ}$ in equation 2.4. The PSF could not be independently measured using a point source placed in air in a zero background due to the nonlinear nature of MAP reconstruction (Qi et al. 1998b). For a line source in air it was determined that a $\beta$ equaling 0.05 resulted in a Gaussian PSF. However, for more distributed sources, such as the mouse heart phantom, this $\beta$ value was not guaranteed to yield a Gaussian PSF. A higher value of $\beta$ was thus chosen to ensure that the PSF could be assumed Gaussian.

As an alternate method, the FWHM of the Gaussian PSF was estimated by changing the resolution in the PV correction algorithm until the non-uniformity (NU) of the mean corrected activity was minimized. The NU is defined by:

$$
\mathrm{NU}=\frac{S D\left(C_{m, 2 D}(\theta)\right)}{\operatorname{Mean}\left(C_{m, 2 D}(\theta)\right)} \times 100 \%
$$

where $S D\left(C_{m, 2 D}(\theta)\right)$ and $M e a n\left(C_{m, 2 D}(\theta)\right)$ are the standard deviation and mean of the PVcorrected activity over all gates (i.e. planes of the physical phantom). Equation 3.1 gives a measure of the overall variability of activity within the phantom and can be applied to uncorrected activity, or to a 3D activity distribution. With uniform activity injected into the fillable portion of the phantom, it was expected that the NU should equal zero. The resolution used in the PV correction algorithm that was found to minimize the NU was thus taken as the best image resolution estimate.

This resolution was used to simulate a $2 \mathrm{D}$ digital phantom that had the same geometry as the physical phantom. Simulations were created by convolving the 2D digital phantom with a 2D Gaussian with the measured FWHM resolution. The PV correction was applied using the same sampling method as described for the measured phantom data. 


\subsection{3. $\left[{ }^{18} F\right]-F D G 2 D$ Mouse Imaging}

Two $30 \mathrm{~g}$ mice were injected with 20 and $35 \mathrm{MBq}$ of $\left[{ }^{18} \mathrm{~F}\right]$-FDG respectively and scanned for 30 minutes, starting 30 -minutes post-injection. Three-dimensional sinograms were binned with 16 ECG gates, and images were reconstructed with OSEM3D/MAP with the same reconstruction parameters as described in Section 3.2.2. The 2D PV correction algorithm was performed on a transverse plane through the LV with the same sampling as discussed in Section 3.2.2. The plane was selected from the central base region. The resolution of the image was estimated by minimizing the $\mathrm{NU}$ of the corrected activity.

\subsubsection{The 1D/3D Partial Volume Correction Algorithm}

The methodology for the 1D/2D PV correction algorithm can be applied to a 3D heart geometry as follows: 3D ECG gated PET images are acquired, and 1D profiles are sampled radially outward from the center of mass of the LV myocardium in 3D (Figure 3.2). In the base and apex regions, sampling is performed in cylindrical $(r, \theta, z)$ and spherical $(r, \theta, \phi)$ coordinates respectively.

All 1D basal and apical profiles are fit to the 1D function as described in Figure 2.1. Apical myocardial, blood and background images are formed by interpolating the fitted ID parameters onto a Cartesian grid. These images are merged with the corresponding base region images and the operations outlined in equations 2.2 and 2.3 are performed to obtain estimates for $I_{P E T, m y o}(g)$ and $I_{\text {conv,myo }}(g)$ in $3 \mathrm{D}$. However, the convolution operation is performed in 3D and the PSF is represented by a 3D Gaussian. The corrected LV myocardium activity $\left(C_{m, 3 D}\right)$ values are sampled in the region of the myocardium along the directions illustrated in Figure 3.2: 


$$
C_{m, 3 D}(\theta,\{z, \phi\}, g)=\frac{I_{P E T, m y o}\left(r=\operatorname{argmax}\left(I_{P E T, m y o}(\theta,\{z, \phi], g), \theta,\{z, \phi\}, g\right)\right.}{I_{\text {conv,myo }}\left(r=\operatorname{argmax}\left(I_{P E T, m y o}(\theta,\{z, \phi], g), \theta,\{z, \phi\}, g\right)\right.}
$$
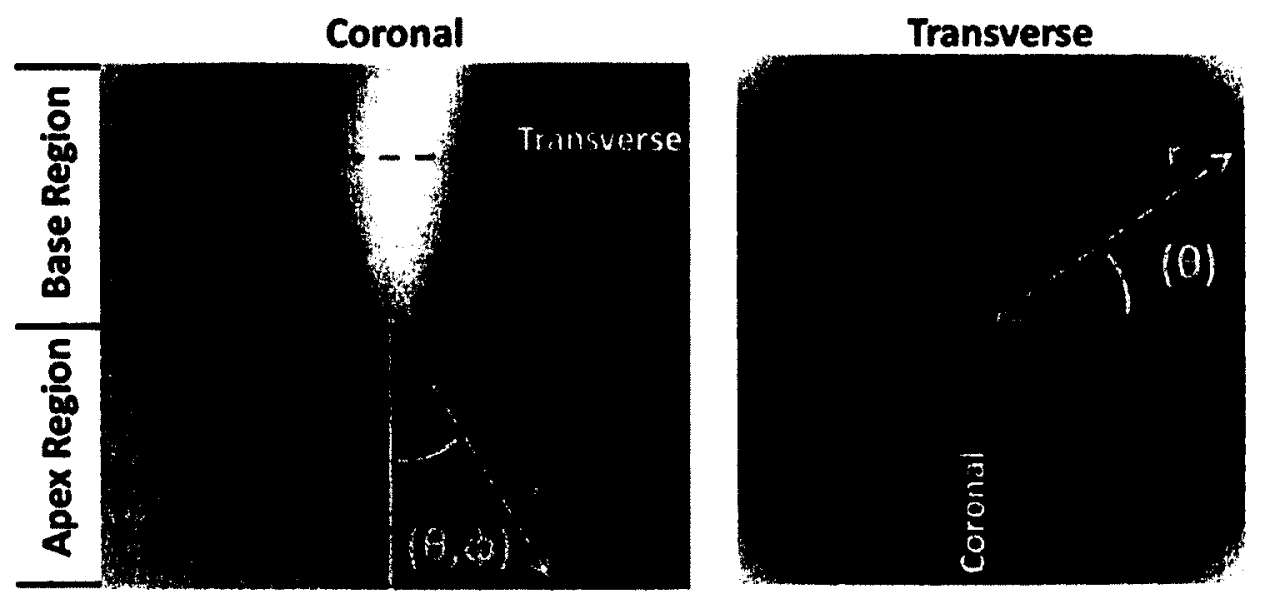

Figure 3.2. Illustration of the 3D sampling process for the base and apex regions on a simulated ECG gated PET image of the heart. The spherical profile sampling $(r, \theta$, $\phi)$ of the apex is shown on the coronal image. The cylindrical $(r, \theta, z)$ sampling performed in the base region is illustrated on the transverse image. The location of each respective image is indicated by the dashed line on the corresponding image.

\subsubsection{The 3D Digital MOBY PET Simulation}

Three-dimensional digital images were simulated using the MOBY phantom convolved with a 3D Gaussian PSF with isotropic resolution and a FWHM equal to the resolution found in Section 3.2.2. The relative activity distribution was typical of an FDG mouse scan at five-minutes post-injection (Table 2.3). The LV activity concentration was set equal to $19 \mathrm{MBq} / \mathrm{mL}$ to be equal to the known activity concentration of the mouse phantom. Simulations were performed on $0.2 \times 0.2 \times 0.4$ and $0.2 \times 0.2 \times 0.8 \mathrm{~mm}^{3}$ pixels, such that 2 axial pixel sizes were considered. Images were linearly interpolated onto 0.1 $\mathrm{mm}$ pixels and the 1D/3D PV correction algorithm was performed using the known FWHM resolution. The $1 \mathrm{D}$ radial profiles and equation 3.2 were sampled with $\Delta \theta=10^{\circ}$, $\Delta \mathrm{z}=0.1 \mathrm{~mm}$ and $\Delta \phi=10^{\circ}$. 


\subsection{Results}

\subsubsection{D/2D PV Correction of the 2D Gated Mouse Heart Phantom}

A resolution of $1.3 \mathrm{~mm}$ FWHM was found to minimize the NU of the PVcorrected activity from the measured phantom data under the assumption of a Gaussian PSF. Figure 3.3 depicts a transverse cross section across the digital, simulated and measured phantom images that were taken at mid-phase between the end-diastole and end-systole phases. Upon comparing Figure 3.3(a) to Figures 3.3(b) and 3.3(c) the PV effects can be clearly observed in both the simulated and measured phantoms. Figures 3.3(b) and (c) do show some small differences and the uncorrected activities differed by $7 \%$ (Table 3.1 ) while the NU differed by approximately $25 \%$. The PV correction algorithm improved the mean LV activity to within $2 \%$ in the case of the simulated data. For the measured data bias in the corrected and true activities were found to be less than $1 \%$ when 150 or 1800 second data was used. With 600 seconds of data, the corrected activity was underestimated by $3 \%$.

For both the measured and simulated data, PV correction improved the NU in all cases. The largest improvement was observed with the simulated data. For the measured data, the NU was improved by approximately $10 \%$ on average with optimal NU being recorded for the longest scan times, which can be attributed to the improvements in count statistics. These results support the ability of the PV correction to improve quantitative accuracy while improving image homogeneity in the 2D phantom. 


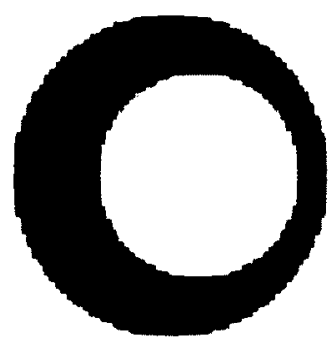

(a)

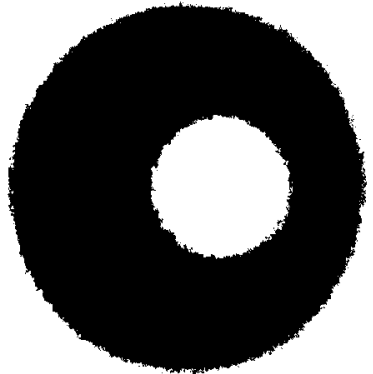

(b)

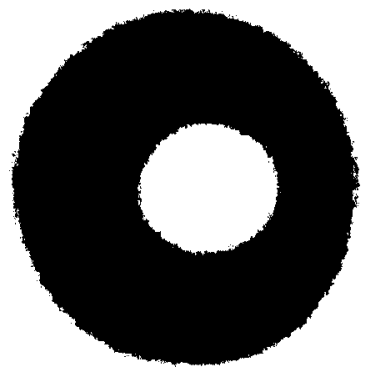

(c)

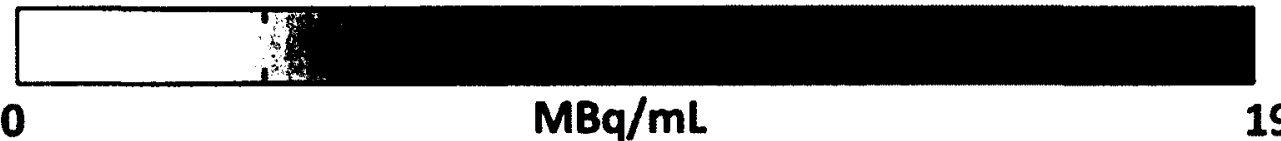

Figure 3.3. Cross section of the mouse heart phantom between the end-systolic and end-diastolic phases: (a) Raw digital phantom image. (b) Simulated image with Gaussian FWHM=1.3 mm and (c) the measured image with $1800 \mathrm{~s}$ of experimental data (OSEM3D/MAP, $\beta=1)$.

TABLE 3.1. 1D/2D PV CORRECTION RESULTS FOR THE 2D SIMULATED AND MEASURED DATA.

\begin{tabular}{ccccc}
\hline \multirow{2}{*}{$\begin{array}{c}\text { Scan Lengths } \\
(\mathrm{s})\end{array}$} & $\begin{array}{c}\text { Mean } \pm \mathrm{SD} \\
(\mathrm{MBq} / \mathrm{mL})\end{array}$ & NU (\%) & $\begin{array}{c}\text { Mean } \pm \mathrm{SD} \\
(\mathrm{MBq} / \mathrm{mL})\end{array}$ & NU (\%) \\
\cline { 2 - 5 } & $11.5 \pm 3.2$ & 28.1 & $19.4 \pm 0.6$ & 3.0 \\
Simulated & $12.2 \pm 2.6$ & 21.5 & $19.0 \pm 2.2$ & 11.9 \\
150 & $12.2 \pm 2.5$ & 20.5 & $18.4 \pm 1.8$ & 9.6 \\
600 & $12.3 \pm 2.4$ & 19.6 & $19.0 \pm 1.3$ & 7.1 \\
1800 & & & $19.0 \pm 0$ & 0 \\
\hline Truth & & & &
\end{tabular}

For the simulated and measured data at 1800 seconds, a graph of activity versus wall thickness is plotted in Figure 3.4. The activity and wall thicknesses are computed as an average across all eight gates, thus each individual point on the graph corresponds to a specific angle $\theta$ through the phantom from the center of the LV cavity. The graphs 
demonstrate the ability of the PV correction algorithm to recover the true activity over a range of wall thicknesses from $0.5-1.5 \mathrm{~mm}$.
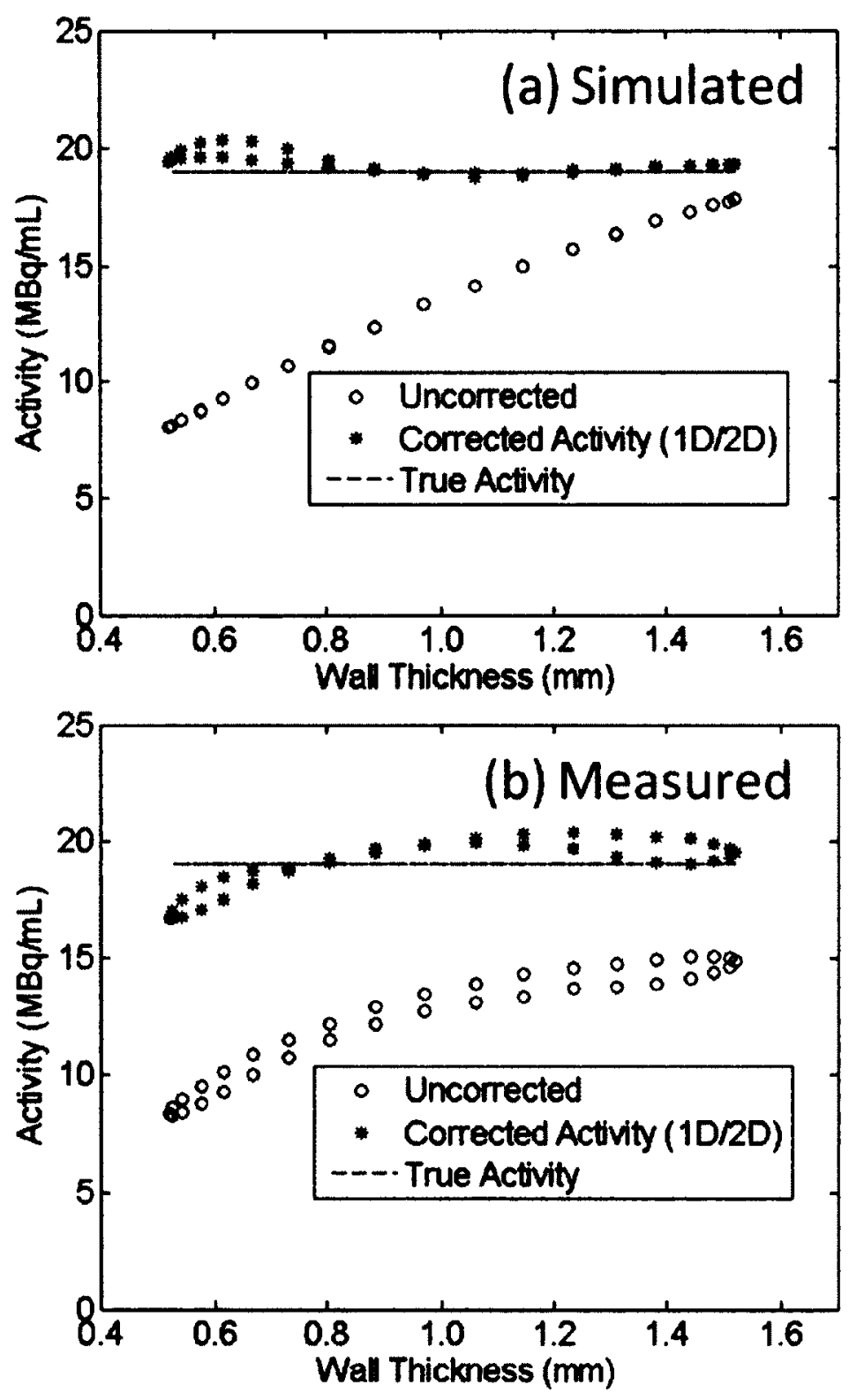

Figure 3.4. Average corrected activity versus average wall thickness for the (a) simulated and (b) measured data with $1800 \mathrm{~s}$ of data. 


\subsection{2. $1 D / 2 D$ PV Correction of $2 D\left[{ }^{18} F\right]-F D G$ Mouse Images}

By minimizing the LV NU, the transverse resolution of the mouse heart images was found to be $1.35 \mathrm{~mm}$, which was comparable to the resolution determined in Section 3.3.1. The results from PV correction of the transverse plane from the $\left[{ }^{18} \mathrm{~F}\right]$-FDG cardiac mouse PET images are shown in Table 3.2.

\section{TABLE 3.2. 1D/2D PV CORRECTION RESULTS FOR THE $\left[{ }^{18} \mathrm{~F}\right]$-FDG CARDIAC MOUSE PET} IMAGES.

\begin{tabular}{ccccc}
\hline \multirow{2}{*}{ Mouse } & \multicolumn{2}{c}{ Uncorrected } & \multicolumn{2}{c}{ Corrected } \\
\cline { 2 - 5 } & $\begin{array}{l}\text { Mean } \pm \mathrm{SD} \\
(\mathrm{MBq} / \mathrm{mL})\end{array}$ & $\mathrm{NU}(\%)$ & $\begin{array}{c}\text { Mean } \pm \mathrm{SD} \\
(\mathrm{MBq} / \mathrm{mL})\end{array}$ & $\mathrm{NU}(\%)$ \\
\hline 1 & $12.1 \pm 1.6$ & 13.5 & $15.8 \pm 1.6$ & 10.2 \\
2 & $34.7 \pm 3.0$ & 8.6 & $41.6 \pm 3.4$ & 8.2 \\
\hline
\end{tabular}

Increases in the LV myocardial activity values were on the order of $30 \%$ and $20 \%$ in mouse 1 and 2 respectively while the NUs were improved by $3.3 \%$ and $0.4 \%$ in the same animals. These results suggest that the RCs in the base region of LV myocardial PET images are approximately $0.7-0.8$. The increased activity values and improved NUs were indicative that the correction was producing similar results as observed for the $2 \mathrm{D}$ mouse phantom. The estimated RCs from the $2 \mathrm{D}$ phantoms with noise were $0.6-0.7$. On average, the $2 \mathrm{D}$ phantom had thinner walls than the imaged mice. Thus, it was expected that the predicted RCs were smaller for the $2 \mathrm{D}$ phantom in comparison to the mice. With both sets of images, corrected NUs were on the order of $10 \%$. 


\subsubsection{D/3D PV Correction of the 3D Digital MOBY PET Simulation}

Results from 1D/3D PV correction of the 3D digital MOBY PET simulations are displayed in Table 3.3. The standard deviation of Table 3.3 is observed to decrease after PV correction because non-uniformities in the activity distribution due to variable wall thickness are minimized. The resolution used for simulation and in the correction algorithm was $1.3 \mathrm{~mm}$, which was equal to the resolution of the $2 \mathrm{D}$ mouse phantom image. In this case the activity was underestimated by $40 \%$ before correction but this was improved to approximately a $10 \%$ underestimation after correction while the NU was improved by $5 \%$. Using an axial pixel size of $0.4 \mathrm{~mm}$ gave superior results in comparison to a $0.8 \mathrm{~mm}$ pixel size in terms of both corrected activity and NU.

Figure 3.5 displays the activity and NU results plotted versus plane selection, which is organized from apex to base for the $0.4 \mathrm{~mm}$ axial pixel size data. Uncorrected and corrected activities from the 1D, 1D/2D and 1D/3D algorithm are shown. The NUs of each corresponding activity value are also plotted. The 1D/2D algorithm was only applicable in the base region of the heart.

TABLE 3.3. 1D/3D PV CORRECTION RESULTS FOR THE 3D SIMULATED PHANTOM.

\begin{tabular}{ccccc}
\hline \multirow{2}{*}{$\begin{array}{c}\text { Axial Pixel } \\
\text { Size }(\mathrm{mm})\end{array}$} & $\begin{array}{c}\text { Mean } \pm \mathrm{SD} \\
(\mathrm{MBq} / \mathrm{mL})\end{array}$ & $\mathrm{NU}(\%)$ & $\begin{array}{c}\text { Mean } \pm \mathrm{SD} \\
(\mathrm{MBq} / \mathrm{mL})\end{array}$ & $\mathrm{NU}(\%)$ \\
\hline 0.4 & $11.9 \pm 1.5$ & 12.8 & $17.3 \pm 1.3$ & 7.6 \\
0.8 & $11.7 \pm 1.6$ & 13.4 & $16.9 \pm 1.4$ & 8.0 \\
\hline
\end{tabular}



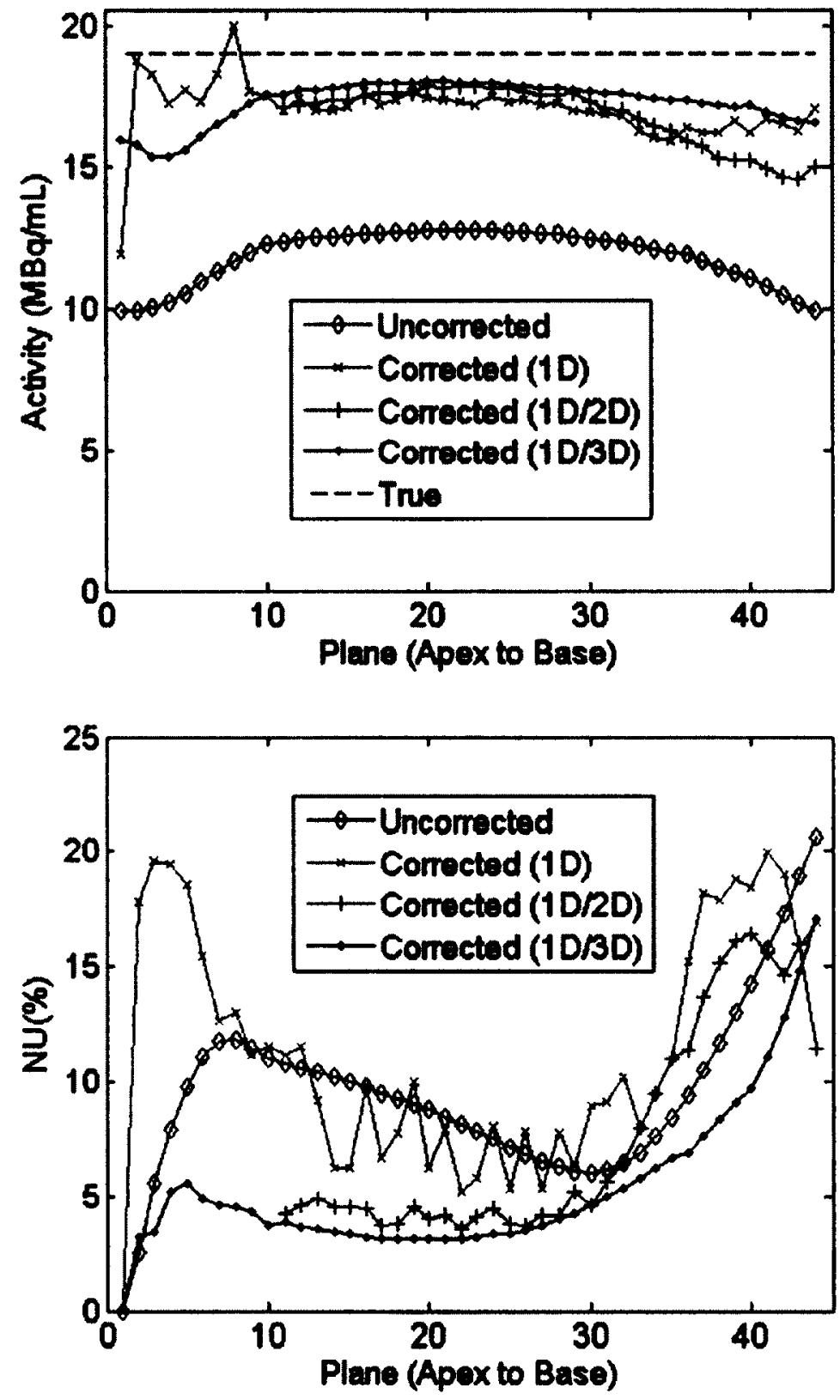

Figure 3.5. Average activity (top) and NU (bottom) versus plane for the 3D simulated MOBY heart phantom. Planes are ordered from apex to base, with the base region starting at plane 11 . The $\mathrm{NU}$ was calculated as the standard deviation divided by the mean activity value. The 1D/2D PV correction algorithm was only performed on slices 11-42. 
The underestimation of activity for the uncorrected data was most pronounced at the apex and base of the heart. The 1D PV correction algorithm improved recovery for all planes. The 1D/2D correction algorithm outperformed the 1D algorithm on average, but these improvements were limited at the base of the heart. The 1D/3D algorithm outperformed the other two algorithms in the base region of the heart. The corresponding plot of NU illustrates that the uncorrected and 1D PV-corrected data had the highest NUs on average over all planes. The 1D/2D algorithm improved the NUs over the planes in the central base region, but the $1 \mathrm{D} / 3 \mathrm{D}$ algorithm had superior $\mathrm{NU}$ over all planes. Although the 1D PV correction had the best recovery in the apex region, the NU was higher than the uncorrected data alone illustrating that this algorithm could not simultaneously improve accuracy while reducing NU. Thus, considering all planes, the 1D/3D PV correction algorithm exhibited the best overall performance.

\subsection{Discussion}

The 1D/2D PV correction algorithm was observed to improve the accuracy $(\mathrm{p}<$ 0.01 ) and $\mathrm{NU}$ of the activity distribution in the measured and simulated 2D phantoms, and in the mouse heart images. The small differences between the measured and simulated phantom images (Figure 3.3(b) and (c)) and activity profiles (Figure 3.4) can be attributed to the nonlinearities of MAP reconstruction (Qi et al. 1998b) and limitations in resolution estimation. OSEM3D/MAP reconstruction depends on the local activity distribution and count statistics. Thus, the resolution properties can change throughout the image. The resolution of the PET image could have also been spatially variant, non- 
isotropic, or non-Gaussian contributing to the residual disagreement. The resolution was determined by assuming a Gaussian function and iterating the PV correction algorithm until the NU was minimized. This FWHM Gaussian resolution value may not accurately describe the blurring of OSEM3D/MAP reconstruction. The uncorrected activity curves for the simulated and measured data (Figure 3.4(a) and (b)) were not identical. In the case of the simulated data, the changes in uncorrected activity between the smallest and largest wall thickness were more pronounced in comparison to the measured data supporting the claimed nonlinear nature of the reconstruction algorithm. The uncorrected activity plot for the measured phantom data also had slightly different values for the same average wall thicknesses (Figure 3.4(b)). This is directly indicative that the resolution properties were changing as a function of position inside the scanner's field. The phantom was not perfectly centered in the field of view. Therefore, the potential spatially variant resolution would result in different resolutions for the different wall thicknesses encountered. These results indicate that further improvements in PV correction may require a more rigorous resolution measurement. In this study, dramatic changes in LV wall thickness were tested in the phantoms. However, mouse wall thicknesses normally vary to a lesser degree (see MOBY phantom cross section in Section 1.11), potentially diminishing these extreme effects.

In the case of the 3D simulated PET image, the 1D/3D algorithm was found to outperform the $1 \mathrm{D}$ and 1D/2D algorithms in terms of simultaneously restoring quantitative accuracy and reducing NU (Figure 3.5). The PV-corrected activity information from the $0.4 \mathrm{~mm}$ pixel size was superior to the $0.8 \mathrm{~mm}$ pixel size using this algorithm. This improved result can be largely attributed to finer sampling, which reduces 
the tissue fraction effect, leading to improved edge detection when performing 1D fitting. This improved quantitative accuracy and image NU.

Although the 1D/3D algorithm was the correction method of choice in the simulation study for 3D imaging, Figure 3.5 revealed that the corrected activity was still underestimated by $15 \%$ in the apex region of the heart suggesting that the proposed methodology still did not fully describe resolution blurring. In the base region of the heart, where the $1 \mathrm{D} / 2 \mathrm{D}$ algorithm was observed to sufficiently perform, the minimal curvature of the heart in the third dimension enabled the ID algorithm to reliably predict local parameters. However, when the 1D algorithm was applied to the spherically curved apex region, such that there was blurring along all three spatial dimensions, the algorithm was unable to predict parameters accurately. Thus the $1 \mathrm{D} / 3 \mathrm{D}$ model was unable to improve results as dramatically as in the base region. It is predicted that these limitations could have been overcome if a complete 3D model was used. Future studies should thus focus on improving the model and resolution limitations outlined here.

\subsection{Conclusion}

The 1D/2D algorithm was observed to restore the accuracy of the activity distribution to within $5 \%$ in the basal region of the heart, while increasing the uniformity in $2 \mathrm{D}$ phantoms and normal mouse hearts. The $1 \mathrm{D} / 3 \mathrm{D}$ algorithm was observed to improve accuracy from a $40 \%$ underestimation to an underestimation of approximately $10 \%$, while also restoring expected image uniformity in simulation studies. Preliminary mouse data measured in vivo supports that the algorithm has the ability for PV correction 
of FDG mouse PET images while simultaneously estimating the 2D transverse resolution. This study provides insight into the limitations of the proposed algorithms for PV correction in mouse PET imaging. To develop a successful cardiac PV correction technique a complete 3D model with true resolution measurements should be considered. 


\section{Chapter 4. A Three-Dimensional Model-Based Partial Volume Correction Strategy for Gated Cardiac Mouse PET Imaging: Principle and Validation}

Preliminary results for the work presented in this chapter were published in abstract form (Dumouchel and deKemp 2011c).

\subsection{Introduction}

The 1D/3D PV correction algorithm developed in Chapter 3 had limited performance in the apex region of the heart because the $1 \mathrm{D}$ model was incapable of describing the $3 \mathrm{D}$ curvature. In this chapter, a completely $3 \mathrm{D}$ five-parameter model is introduced that is predicted to overcome the limitation of the $1 \mathrm{D} / 3 \mathrm{D}$ algorithm. The model is similar to the $1 \mathrm{D}$ fitting technique, except that an entire $3 \mathrm{D}$-heart geometry is considered in the model. The algorithm is tested on simulated and measured phantom 
data with an activity distribution based on FDG cardiac mouse PET imaging. The PV correction algorithm is also performed on measured cardiac mouse PET images. Ex vivo biodistribution is performed for mouse imaging, in order to compare PV-corrected data to the true activity distribution. Quantitative calibration is performed by using the liver as a reference organ, such that accurate image calibration is performed for each mouse. The PSF is also measured for each individual mouse image using a point source to consider the nonlinear nature of the reconstruction. Similar to the previous 1D/2D and 1D/3D algorithms, the method presented in this chapter is not dependent on kinetic modeling and cardiac motion is eliminated by ECG gating.

\subsection{Materials and Methods}

\subsubsection{The Partial Volume Correction Algorithm}

The PV correction algorithm is based on a 3D geometrical model of the activity distribution in the LV myocardium convolved with the 3D scanner PSF. The estimated activity distribution in the LV is described by blood $\left(A_{\text {blood }}\right)$, background $\left(A_{\text {back }}\right)$ and myocardial $\left(A_{m y o}\right)$ activities which are separated into three distinct compartments by the endocardial radius $(R)$ and the myocardial wall thickness $(w)$. Each parameter is varied regionally, as a function of myocardial position within the 3D volume. An estimated PET image $\left(E_{P E T, 3 D}\right)$ is computed as the convolution between the modeled activity distribution $\left(I_{L V, 3 D}\right)$, as defined by the five parameters, and a 3D PSF $\left(P S F_{3 D}\right)$ :

$$
E_{P E T, 3 D}=I_{L V, 3 D} \otimes P S F_{3 D} .
$$


The sum of squared error is computed between $E_{P E T, 3 D}$ and a measured ECG gated PET image (MPET,3D). The regional parameters $A_{\text {blood }}, A_{b a c k}, A_{m y o}, R$ and $w$ are adjusted using the method of nonlinear least squares to minimize the sum of squared error between $E_{P E T, 3 D}$ and $M_{P E T, 3 D}$ as illustrated in Figure 4.1. ECG-gated PET images are acquired to minimize cardiac motion and associated PV effects, and the algorithm is performed individually on each gate $(g)$. The regional parameters are sampled radially from the center of mass of the myocardium. In the base region the parameters are sampled cylindrically $(r, \theta, z)$ while in the apex region, the parameters are sampled spherically $(r, \theta, \phi)$. Initial parameter estimates for $A_{\text {blood, }} A_{b a c k}, R$ and $w$ are obtained using the 1D model that assumes a profile through the myocardium can be described by a 1D square wave convolved with a 1D Gaussian as described in Chapter 2 (Figure 2.1). The initial estimate for $A_{m y o}$ is equal to the maximum value in the LV myocardial region of $M_{P E T, 3 D}$.

Within each iteration, the complete set of regional parameters is updated using the method of nonlinear least squares. Local regularization of $A_{\text {myo }}, A_{\text {back }}, R$ and $w$ is performed to enforce smoothness of the myocardial contours and activity distribution. $A_{\text {blood }}$ is taken as an average over all the sampled blood regions assuming that the activity in the blood is uniform. Local parameters are updated with 10 nonlinear least squares iterations. The algorithm is implemented in MATLAB using 20 iterations of the full PV correction model. Convergence of the algorithm was assessed using simulated phantom data as described in Section 4.2.3. 


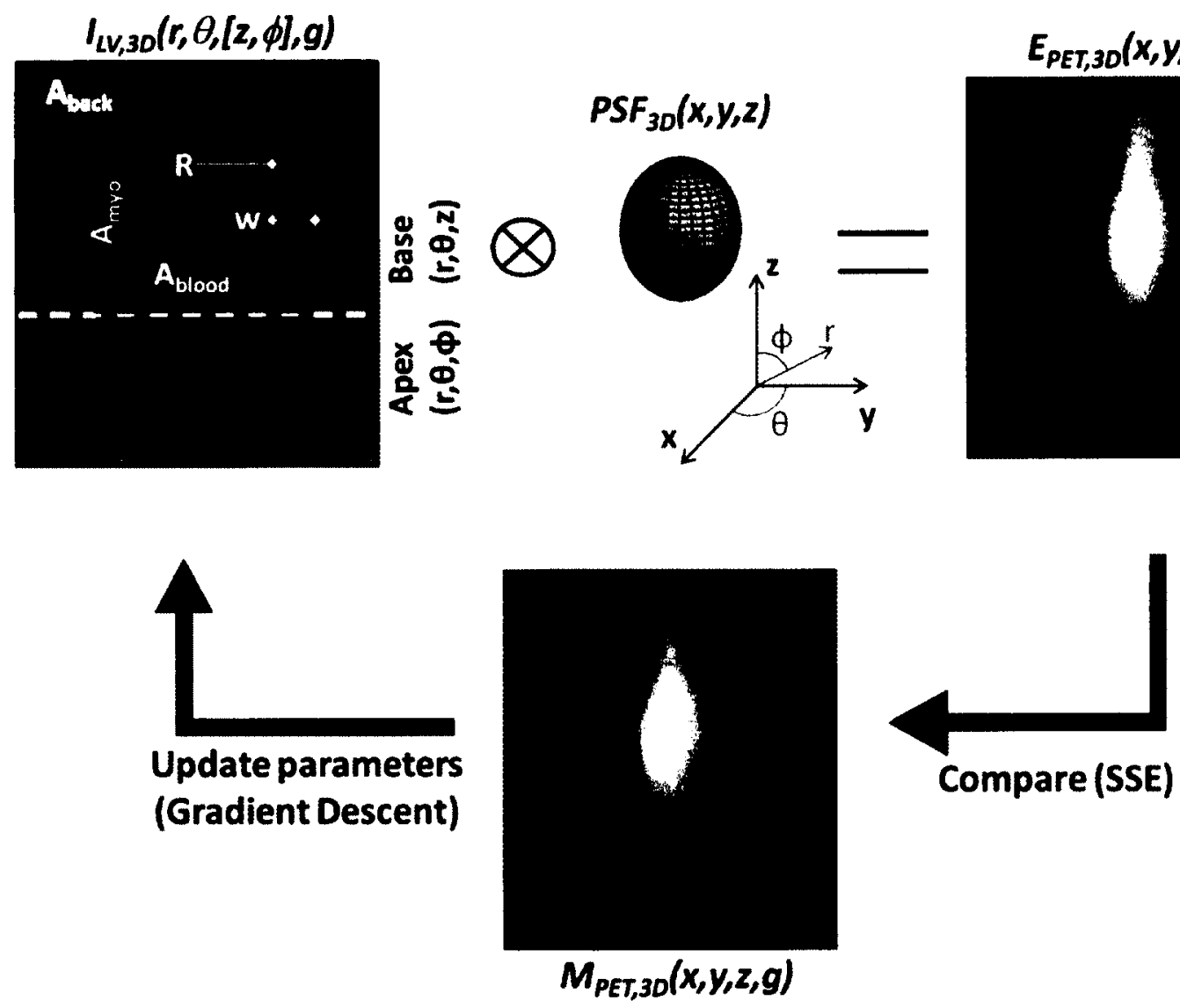

Figure 4.1. Schematic illustration of the fully 3D PV correction algorithm.

The basal transverse plane is chosen to ensure that there is myocardial activity measured in all radial profiles around $360^{\circ}$ (i.e. at the basal septum). Above this plane, $I_{L V, 3 D}$ is approximated by inserting $M_{P E T, 3 D}$ to account for the missing data in the model. 
Angular sampling in the base and apex regions is performed by varying $\theta$ and $\phi$ in $30^{\circ}$ increments. A total of 9 planes define the axial extent of the $\mathrm{LV}$ in the base region. The PSF represents the resolution blurring due to position decoding accuracy, scanner detector size, positron range, scanner diameter and the reconstruction effects Moses and Derenzo 1993). It is computationally intensive to convolve a 3D PSF with $I_{L V, 3 D}$ for each iteration. Thus, $P S F_{3 D}$ is assumed to be a 3D Gaussian described by transverse $(\mathrm{x}, \mathrm{y})$ and axial (z) full width at half maximum (FWHM) values, so the convolution operation can be implemented as a series of separable 1D convolutions.

\subsubsection{Mouse PET Imaging and Biodistribution}

Mouse PET imaging was performed with the Inveon PET scanner. Normal Friend Leukemia Virus $B$ (FVB) mice $(N=10)$ were anaesthetized under $2 \%$ isoflurane with the heart centered in the field of view. A ten-minute transmission scan, with an energy window set to $120-125 \mathrm{keV}$, was acquired using a rotating ${ }^{57} \mathrm{Co}$ source with $95 \mathrm{MBq}$ of activity. $30-42 \mathrm{MBq}$ of $\left[{ }^{18} \mathrm{~F}\right]-\mathrm{FDG}$ was administered through the tail vein and list-mode data were acquired for 60 minutes using energy and timing windows of $350-650 \mathrm{keV}$ and 3.42 ns respectively. An ECG gating trigger was simultaneously acquired using the Model 1025T small animal monitoring and gating system (SA Instruments Inc., Stony Brook, NY). Emission list-mode data were binned into two sets of 3D sinograms with a ring difference of 79 and a span of 3 . The first set of sinograms consisted of the final 30 minutes of data divided into eight equally spaced ECG gates based on heart-rate adaptive binning. The second set of sinograms, used for accurate normalization to the scan endtime, consisted of the final five minutes of data with no gating. All images were reconstructed using OSEM3D/MAP algorithm with 2 OSEM3D iterations, 18 MAP 
iterations and $\beta$ set to 0.5 , to ensure a Gaussian PSF (see Section 3.2.2), with uniform resolution and fastMAPTM enabled. The images were reconstructed onto a $256 \times 256 \times$ 159 matrix with $0.22 \times 0.22 \times 0.80 \mathrm{~mm}^{3}$ voxels. Randoms subtraction was performed using the delayed coincidence window technique while detector efficiencies were corrected using a component-based normalization. Data were also corrected for isotope decay and detector dead-time. Images were reconstructed with and without scatter and attenuation corrections applied to examine the effect of these phenomena. Scatter correction was performed using the single scatter simulation and attenuation correction was based on the transmission scan (see Section 1.7).

Following imaging at 60 minutes post-injection, the mice were sacrificed and the RV myocardium, LV myocardium, liver, LV blood, lung, aorta, left atrium and right atrium were extracted. The masses of the tissue samples were measured and a Packard Cobra II gamma counter (Perkin Elmer, Boston, MA) was used to determine the $\left[{ }^{18} \mathrm{~F}\right]-$ FDG activity present in each tissue, expressed as a percent injected dose per gram $(\% / g)$; defined as the total activity in the tissue sample per gram divided by the injected activity. Animal experiments were performed in agreement with the guidelines set forth by the University of Ottawa Animal Care Committee and approved by the Canadian Council on Animal Care.

The PV correction as illustrated in Figure 4.1 was applied to the 30-minute gated images with and without correction for scatter and attenuation. Due to the nonlinearity of the OSEM3D/MAP reconstruction algorithm, the resolution of the reconstructed images could not be estimated by placing a point source in air (Qi et al. 1998b). Instead, a ${ }^{22} \mathrm{Na}$ point source with a $0.25 \mathrm{~mm}$ diameter and approximately $1 \mathrm{kBq}$ of activity that was 
embedded in a $1 \mathrm{~cm}^{3}$ cube of acrylic was placed in the scanner at positions corresponding to the location of the LV myocardium in the mouse PET images. Although the positron ranges of ${ }^{18} \mathrm{~F}$ and ${ }^{22} \mathrm{Na}$ differ, it has been shown that the distribution of positron ranges between the two isotopes are similar enough to justify the use of ${ }^{22} \mathrm{Na}$ for resolution measurements (Alessio et al 2010b). Five-minute acquisitions were performed with the point source located within the myocardial contours along each of the cardinal directions as illustrated in Figure 4.2. This process was repeated for the end-diastolic and endsystolic images yielding a total of ten resolution measurements per mouse.

The point source emission data were binned into 3D sinograms as described for the mouse studies. The point source sinograms were scaled prior to being added to the mouse sinograms to ensure that only a small perturbation was introduced (i.e. $<1 \%$ of the counts in each sinogram bin) into each mouse sinogram such that the reconstructed image was minimally affected (Fessler and Rogers 1996). The perturbed sinograms were reconstructed using OSEM3D/MAP using the same parameters as described above, and the difference between the mouse images and the perturbed mouse images was computed to obtain the local PSF. A 3D Gaussian function was fit to each difference image using the method of nonlinear least squares. The radial and axial PSF FWHM resolutions were extracted from the base and apex regions respectively. An estimated position-independent transverse resolution $(\mathrm{x}, \mathrm{y})$ was obtained by averaging all of the radial resolution estimates over all positions and gates for each mouse. The position-independent axial resolution (z) was computed as the mean of the two apex measurements at end-diastole and end-systole. 

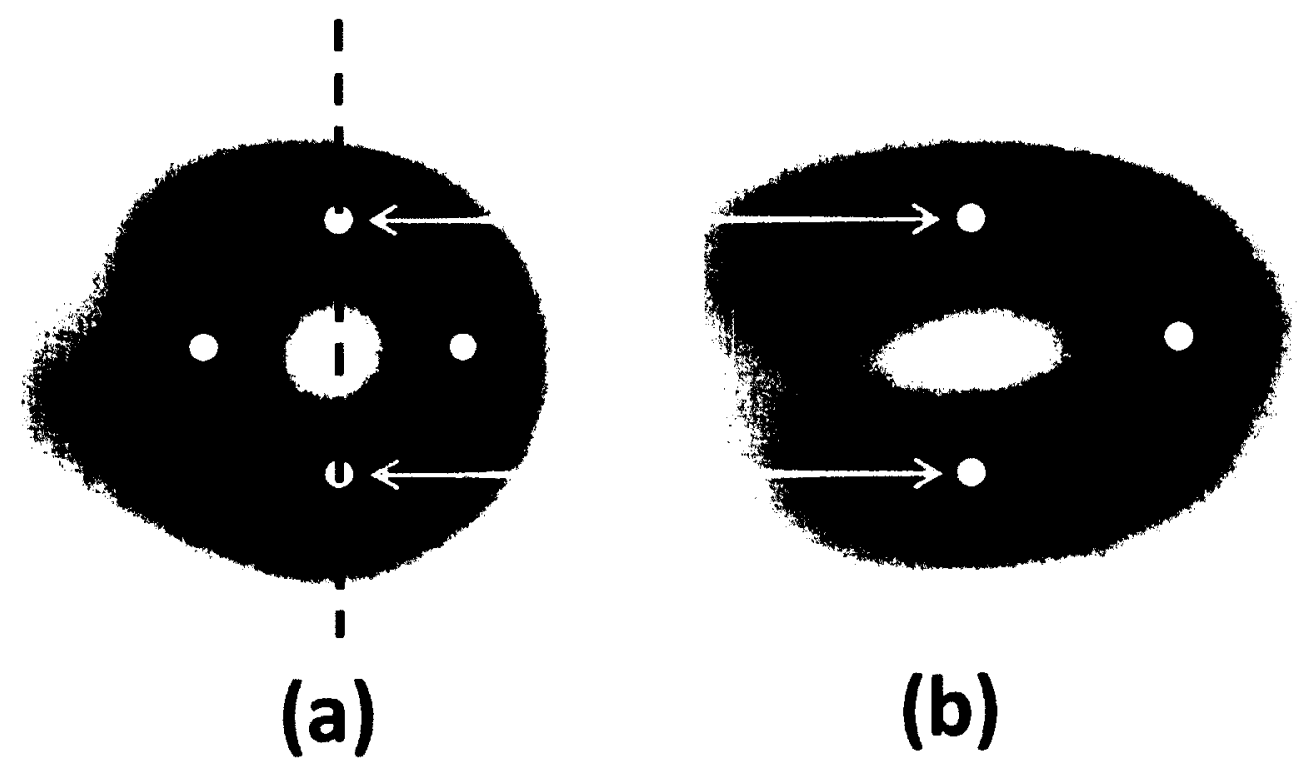

Figure 4.2. Illustration of the location of the point sources in the base and apex regions on an end-diastolic PET image of a $28 \mathrm{~g}$ mouse injected with $32 \mathrm{MBq}$ of $\left[{ }^{18}\right.$ F]-FDG. (a) Four point source measurements were obtained along the cardinal directions on one mid-LV plane. (b) One point source measurement was taken at the apex. The point source locations are indicated by the white dots. The dashed black line on image (a) shows the location of the cross section for image (b). The gray arrows indicate the point source location on each respective image.

For the initial 1D estimate algorithm, the measured resolution along the 1D profile was taken as the transverse resolution in the base region. However, in the apex region this resolution was based on a combination of the transverse and axial resolution estimates, depending on the sampling angle $(\phi)$ of the profile. The 3D PV correction algorithm was performed on the 30-minute gated mouse images using these estimated resolutions and the initial estimates from the $1 \mathrm{D}$ algorithm. 
The gamma counter and PET scanner were both calibrated using $\left[{ }^{18} \mathrm{~F}\right]$-FDG measured in a Capintec CRC-15R (Capintec Inc., Ramsey, NJ) with a calibration number setting of 439 . A $40 \mathrm{MBq}, 0.1 \mathrm{~mL}$ sample was placed in a $1 \mathrm{~mL}$ syringe $5 \mathrm{~cm}$ from the bottom of the chamber and centered in the transverse plane. The activity was diluted into $100 \mathrm{~mL}$ of water, and $1 \mathrm{~mL}$ of the solution was measured inside the gamma-counter to determine the calibration constant of the gamma-counter ( $\mathrm{cpm} / \mathrm{Bq})$. The PET scanner was calibrated by diluting another $40 \mathrm{MBq}, 0.1 \mathrm{~mL}$ of FDG into a $30 \mathrm{~mL}$ volume sample that was placed into a $60 \mathrm{~mL}$ syringe. The syringe was scanned for two hours and the data reconstructed using the same parameters described for mouse imaging. Both methods were subject to errors due to volume measurements and to the precise source geometry of the initial and residual activities inside the dose calibrator. PET image calibration was dependent on the activity distribution and reconstruction parameters. To avoid potential calibration bias, a reference organ was also used to calibrate the measured activities between the gamma counter and PET scanner. The liver was selected as the calibration organ because there was sufficient FDG uptake at 60 minutes post-injection and a VOI could be drawn reproducibly within the central region such that liver activity PV losses could be avoided. A $0.3 \mathrm{~cm}^{3}$ VOI was drawn over the liver region on the five-minute ungated image $\left(A_{\text {liver,imgs }}\right)$. This factor was used in combination with the measured liver activity from the biodistribution $\left(A_{\text {liver, biod }}\right)$ to normalize PET myocardial activity to the biodistribution activity scale as shown in equation 4.2. Such organ-based calibration thereby converted the measured PET image activity $(\mathrm{MBq} / \mathrm{mL})$ directly into the same units as the biodistribution activity $(\mathrm{MBq} / \mathrm{g})$ avoiding potential bias from inaccurate calibration of PET or gamma-counter instruments. The PV-corrected image activity 
$\left(\hat{A}_{m y o, i m g 30}\right)$ was obtained from the 30 -minute gated studies. The uncorrected activities from the five-minute $\left(A_{m y o, i m g}\right)$ and the 30 -minute $\left(A_{m y o, i m g 30}\right)$ images were used to adjust $\hat{A}_{m y o, i m g 30}$ to be comparable to the end-scan activity from the late five-minute image:

$$
\hat{A}_{m y o, b i o d}\left[\frac{M B q}{g}\right]=\hat{A}_{m y o, i m g 30}\left[\frac{M B q}{m L}\right] \times \frac{A_{m y o, i m g 5}}{A_{m y 0.1 m g 30}}\left[\frac{1}{1}\right] \times \frac{A_{l i v e r, b i o d}}{A_{\text {liver, }, m g s}}\left[\frac{m L}{g}\right]
$$

where $\hat{A}_{m y o, b i o d}$ was the calibrated PV-corrected activity (MBq/g). This activity could be directly compared to the LV myocardial activity from the biodistribution $\left(A_{\text {myo,biod }}\right)$ under the assumption that the densities of tissue in the liver and myocardium are equal in mice. The comparison also assumes that the final five-minute measurement end-frame is representative of the biodistribution activity obtained at the time of sacrifice.

The calibrated PV-uncorrected activity was computed using equation 4.2 by setting $\hat{A}_{m y o, i m g 30}$ and $A_{m y o, i m g 30}$ equal to unity. LV myocardial activity RC values were calculated by dividing PV-uncorrected and PV-corrected activities by $A_{\text {myo,biod. }}$ The NU of the PV-uncorrected and PV-corrected myocardial activity polar map was calculated as the SD divided by the mean and expressed as a percent. Polar map images were generated using flowQuant (Klein et al. 2010). Operations were performed on the image data with and without corrections for attenuation and scatter. Paired t-tests were used to compare PV-corrected versus uncorrected values, and to compare values with versus without scatter and attenuation correction. A p-value $<0.05$ was considered statistically significant.

\subsubsection{D Digital Simulations}

3D digital simulations were created using the MOBY phantom. The mean organ activity values from the biodistribution for the RV myocardium, LV myocardium, liver, LV blood, lung, aorta, left atrium and right atrium were averaged over all mice and these 
values were used to set the activity distribution of the gated MOBY phantom $\left(I_{M O B Y, g}\right)$. A total of eight ECG gates were simulated. Simulated mouse PET images $\left(I_{\text {Sim, }}\right)$ were generated with and without noise using the following equation:

$$
l_{S i m, g}=\left\{l_{M O B Y, g} \times[1+N(0,1) \times S D(A)]\right\} \otimes P S F_{G a u s s, 3 D}
$$

where $g$ is gate number, $N(0,1)$ are normally distributed random numbers and $S D$ is the standard deviation used to adjust the magnitude of additive noise as a function of activity (A). The noise was added to the image prior to the convolution with a 3D Gaussian PSF $\left(P S F_{\text {Gauss,3D) }}\right.$ such that the noise in the resulting image was spatially correlative (Aston $e t$ al. 2002). In other words, the reconstruction PSF blurs the noise in the raw data. The simulated images were generated to be comparable to the OSEM3D/MAP reconstructed image from the Inveon and it has previously been shown that the correlative noise dominated over uncorrelated noise (Section 2.3.2). To simulate the appropriate amount of noise, a $30 \mathrm{~mL}$ syringe with a $2.4 \mathrm{~cm}$ diameter was filled with $22 \mathrm{~mL}$ of an $\left[{ }^{13} \mathrm{~N}\right]$-solution that had $50 \mathrm{MBq}$ of activity. The syringe was scanned for 90 minutes with a $350-650$ $\mathrm{keV}$ energy window and a $3.42 \mathrm{~ns}$ timing window. The data were binned into $3 \mathrm{D}$ sinograms in ten-minute intervals and reconstructed with the same parameters as described for mouse imaging. A cylindrical VOI was drawn on the reconstructed image that had a $0.9 \mathrm{~cm}$ radius and a $4 \mathrm{~cm}$ length and the mean and standard deviations voxel values were calculated. The squared SNR was plotted versus the relative activity concentration. A linear fit was performed and this relationship was used to adjust the Gaussian noise, proportional to activity, added to $I_{M O B Y, g}$ as previously described (Section 2.2.4). The SNR of liver VOIs from the mouse studies (Section 4.2.2) was computed and averaged. The squared SNRs for other organs were calculated using the linear fit with the 
measured relative activity concentrations from the biodistribution data. The transverse and axial FWHM resolutions used in the simulations were computed as the average resolutions over all mice from the mouse biodistribution study. One gated image set was generated without noise while five gated image sets were generated with noise. The PV correction algorithm was applied to the data using the known simulated resolution.

\subsubsection{A Mouse Phantom}

A 3D LV mouse phantom was designed to validate the algorithm performance. The phantom was constructed from clear cast acrylic and had two fillable chambers which represented the liver and LV myocardium at end-diastole. The phantom was cylindrical and had the same outer dimensions as the NEMA mouse-like phantom (National Electrical Manufacturers Association 2008) with a $2.5 \mathrm{~cm}$ outer diameter and a $7 \mathrm{~cm}$ length. The LV wall thickness was estimated from echocardiography (Youn et al. 1999, Gardin et al. 1995, Collins et al. 2001, Pollick et al. 1995, Gao et al. 2000, Rottman et al. 2007) and the MOBY phantom (Segars et al. 2000) so the average wall thickness at end-diastole was $0.83 \mathrm{~mm}$. The apex-to-base length of the myocardium was approximated from (Youn et al. 1999, Collins et al. 2001, Segars et al. 2000) as $6.72 \mathrm{~mm}$ and the LV cavity was estimated to be $3.38 \mathrm{~mm}$ at end-diastole (Youn et al. 1999, Gardin et al. 1995, Collins et al. 2001, Pollick et al. 1995, Rottman et al. 2007,Segars et al. 2000). The distance from the chest wall to the center of the LV cavity and the distance from the head to the top of the LV myocardium were derived from the mouse PET imaging described in Section 4.2.2; estimated to be $5.85 \mathrm{~mm}$ and $29.7 \mathrm{~mm}$ respectively. The LV myocardial chamber was designed such that the base region was cylindrical and the apex region was hemispherical. Two $1 \mathrm{~mm}$ diameter ports were situated above the 
base region of the LV to allow for fluid filling and air escape. The liver was positioned 3 $\mathrm{mm}$ below the bottom of the $\mathrm{LV}$ myocardial chamber and $2.5 \mathrm{~mm}$ from the outside wall to ensure structural integrity. The liver compartment was approximated by a cylinder with a height of $7.5 \mathrm{~mm}$, which was based on the mouse PET images. The mass of a mouse liver for a $30 \mathrm{~g}$ mouse was found to be roughly $1.5 \mathrm{~g}$ (Boxenbaum 1980). Under the assumption that the density of the liver is $1 \mathrm{~g} / \mathrm{mL}$, the diameter of the liver cavity was calculated as $1.6 \mathrm{~cm}$. A $2 \mathrm{~mm}$ hole was positioned to allow for liver activity filling and air escape. The phantom design is illustrated in Figure 4.3. To emulate background activity, a separate phantom was constructed that had the same outer dimensions as the LV phantom but had a hollow fillable region. The wall thickness of the phantom was 2.5 $\mathrm{mm}$ while the caps at either end of the cylinder were $5 \mathrm{~mm}$ thick.

A ten-minute transmission scan, with an energy window set to $120-125 \mathrm{keV}$, was acquired for the LV mouse phantom using the ${ }^{57} \mathrm{Co}$ source. The LV and liver chambers were filled with $11 \mathrm{MBq} / \mathrm{mL}$ of $\left[{ }^{18} \mathrm{~F}\right]-\mathrm{FDG}$ and the hollow phantom was filled with 1 $\mathrm{MBq} / \mathrm{mL}$. Each phantom was imaged with the Inveon for 30 minutes with energy and timing windows of $350-650 \mathrm{keV}$ and $3.42 \mathrm{~ns}$ respectively. The data were binned into $3 \mathrm{D}$ sinograms with a ring difference of 79 and a span of 3 . The known activity concentrations in each phantom were used to temporally adjust how much of the list-mode data was to be binned so that the count statistics in the sinograms resembled the gated cardiac mouse heart imaging described in Section 4.2.2. Therefore, the number of counts in the LV mouse phantom sinogram was selected to be equal to the number of counts as if the average LV activity concentration from the biodistribution had been injected into the phantom and imaged for the same length of time as one of the gates (225 seconds). 
Similarly, the number of counts binned into the hollow phantom sinogram was based on the average blood activity concentration from the biodistribution. The count-adjusted sinograms were added together and reconstructed with the same parameters as described for mouse imaging. The reconstruction was repeated with and without attenuation and scatter correction applied. The transverse and axial resolutions of the phantom image were determined following the perturbative approach as described in Section 4.2.2. The phantom only had one cardiac phase. To improve the number of transverse resolution estimates in the base, 4 measurements along the cardinal directions were repeated on two different transverse planes that were separated by $1.6 \mathrm{~mm}$ axially. One measurement was made at the apex. The activities in the liver and myocardium chambers were known to be equal. A VOI was drawn over the central region of the liver compartment with a $5 \mathrm{~mm}$ radius and a $4 \mathrm{~mm}$ height to estimate the mean activity in the liver chamber. This activity was compared to the activity in the LV myocardium to estimate RC.

A digital phantom was created that had the same dimensions as the LV mouse phantom. The activity distribution was set to equal the activity distribution of the imaged phantom. A simulated image was generated by convolving the digital image with the scanner 3D Gaussian PSF, with transverse and axial FWHM resolutions determined from the perturbation measurements of the measured LV mouse phantom. The PV correction algorithm was applied to the measured and simulated data with the estimated and known PSFs respectively. 


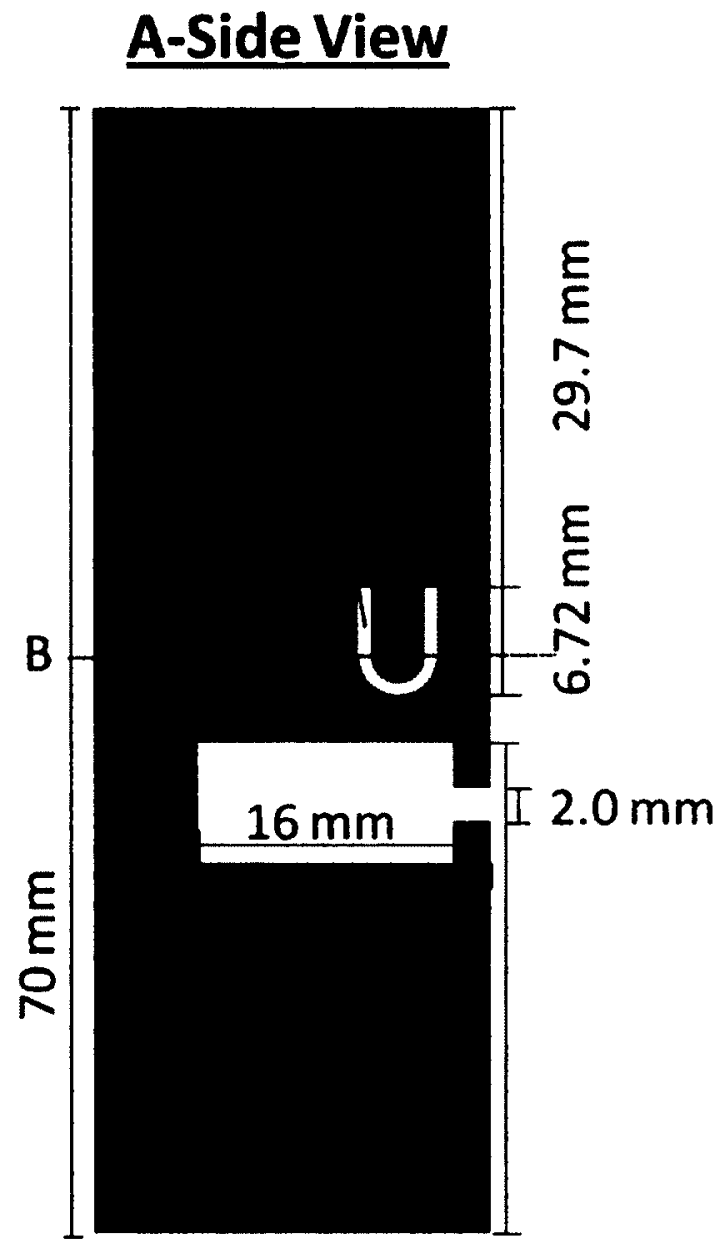

\section{B-Cross Section}

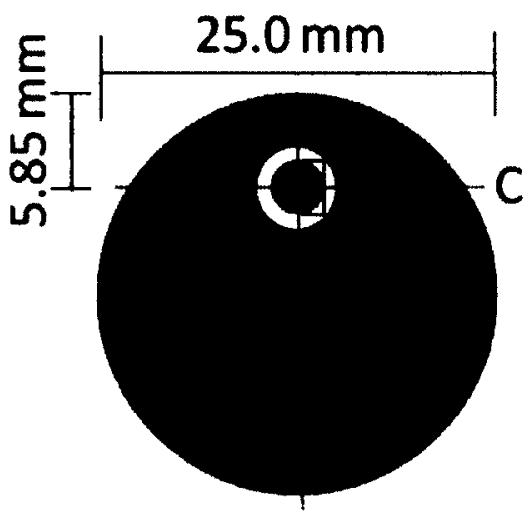

\section{C-Side View (truncated)}

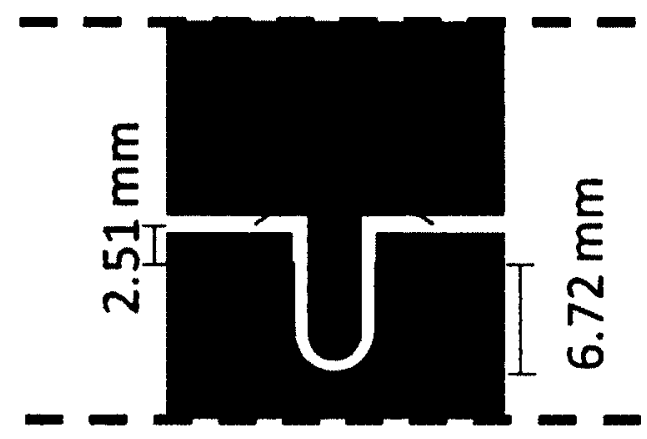

Figure 4.3. The end-diastolic LV mouse phantom with fillable LV and liver chambers. Notable dimensions are shown on the figure. View $A$ illustrates a side view through the phantom that intersects through middle of the $\mathrm{LV}$ and liver chambers. View $B$ is a cross section of the $L V$ region to demonstrate the $L V$ dimensions. The dashed lines of view $\mathbf{C}$ indicate that only a portion of the phantom was selected. The purpose of view $C$ is to show the injection ports for the $L V$. 


\subsection{Results}

\subsubsection{Resolution of Iterative Reconstruction}

Figure 4.4 illustrates a 1D radial profile and the associated fitted line for a PSF obtained from the perturbation of a reconstructed mouse image. The average transverse and axial FWHM resolutions obtained from the mouse imaging are displayed in Table 4.1 with the associated SDs. The mean transverse and axial resolution measurements were $1.45 \pm 0.03$ and $2.10 \pm 0.05 \mathrm{~mm}$ respectively corresponding to NUs of 2.1 and $2.4 \%$.

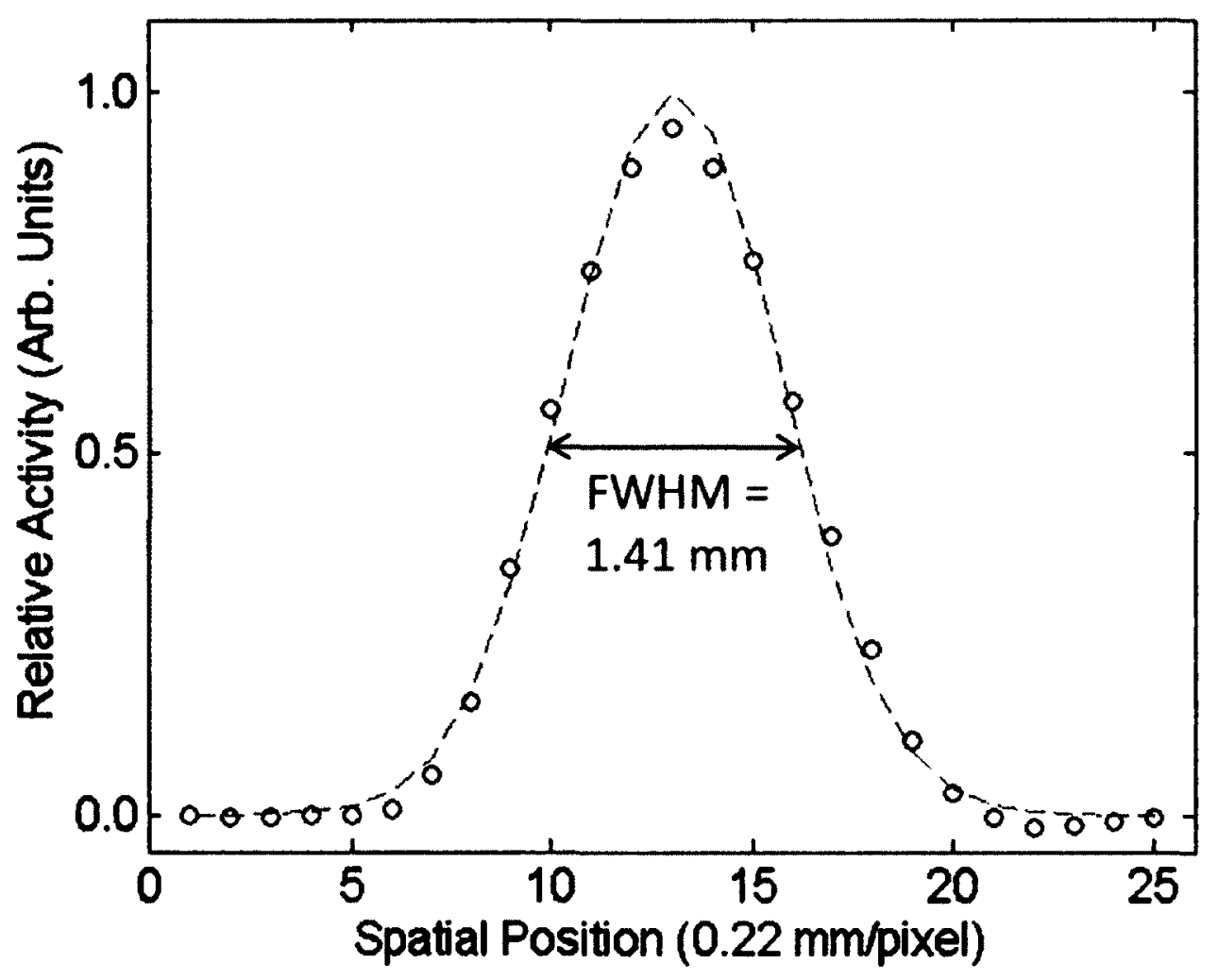

Figure 4.4. Example radial profile across a measured PSF (open circles) with the fitted profile (dashed line). The data shown were taken from mouse 4 at end-diastole with the point source placed at the basal lateral wall location. 
TABLE 4.1. MEAN (SD) TRANSVERSE AND AXIAL FWHM RESOLUTION MEASUREMENTS.

\begin{tabular}{|c|c|c|}
\hline Mouse & $\begin{array}{c}\text { Transverse } \\
\text { (mm) }\end{array}$ & Axial (mm) \\
\hline 1 & $1.48(0.03)$ & $2.14(0.05)$ \\
\hline 2 & $1.48(0.02)$ & $2.20(0.01)$ \\
\hline 3 & $1.50(0.08)$ & $2.12(0.05)$ \\
\hline 4 & $1.41(0.03)$ & $1.88(0.04)$ \\
\hline 5 & $1.45(0.02)$ & $2.12(0.07)$ \\
\hline 6 & $1.44(0.04)$ & $2.15(0.01)$ \\
\hline 7 & $1.44(0.04)$ & $2.07(0.04)$ \\
\hline 8 & $1.43(0.02)$ & $2.17(0.03)$ \\
\hline 9 & $1.45(0.03)$ & $2.01(0.08)$ \\
\hline 10 & $1.41(0.03)$ & $2.10(0.10)$ \\
\hline Mean $\left(\mathrm{SD}_{\mathrm{avg}}{ }^{*}\right)$ & $1.45(0.03)$ & $2.10(0.05)$ \\
\hline
\end{tabular}

\subsubsection{Biodistribution}

The mean activity from the liver VOIs for each five-minute ungated mouse image is summarized in Table 4.2. The mean biodistribution data across all mice for the LV myocardium, RV myocardium, liver, lung, LV blood, aorta, right atrium and left atrium are given in Table 4.3. The mean percent injected dose per gram was calculated for each tissue and multiplied by $33 \mathrm{MBq}$ as a representative injected dose. The biodistribution data were converted from $\mathrm{MBq} / \mathrm{g}$ to $\mathrm{MBq} / \mathrm{mL}$ by assuming a soft tissue density of $1 \mathrm{~g} / \mathrm{mL}$ and a density of $0.26 \mathrm{~g} / \mathrm{mL}$ for lung, which is the density of inflated human lung tissue (Snyder et al. 1975). 
TABLE 4.2. MEAN (SD) $\left[{ }^{18}\right.$ F]-FDG UPTAKE IN THE LIVER 55 MINUTES POST-INJECTION.

\begin{tabular}{cccc}
\hline Mouse & $\begin{array}{c}\text { Liver } \\
(\mathrm{MBg} / \mathrm{mL})\end{array}$ & $\begin{array}{c}\text { Liver } \\
\text { NU }(\%)\end{array}$ & $\begin{array}{c}\text { SNR } \\
(\text { Mean/SD) }\end{array}$ \\
\hline 1 & $0.87(0.11)$ & 12.7 & 7.8 \\
2 & $0.65(0.10)$ & 14.9 & 6.7 \\
3 & $0.75(0.09)$ & 12.3 & 8.1 \\
4 & $0.67(0.10)$ & 14.6 & 6.8 \\
5 & $0.77(0.08)$ & 10.6 & 9.4 \\
6 & $0.70(0.08)$ & 11.7 & 8.6 \\
7 & $0.69(0.09)$ & 13.3 & 7.5 \\
8 & $0.63(0.08)$ & 12.2 & 8.2 \\
9 & $0.60(0.07)$ & 12.4 & 8.1 \\
10 & $0.91(0.19)$ & 21.0 & 4.8 \\
\hline Mean & $0.72(0.10)$ & 13.6 & 7.6 \\
\hline
\end{tabular}

TABLE 4.3. MEAN (SD) BIODISTRIBUTION VALUES TAKEN OVER THE 10 MICE AT 60 MINUTES POST-INJECTION FOR VARIOUS TISSUES.

\begin{tabular}{cccccccc}
\hline $\begin{array}{c}\mathrm{LV} \\
\text { myocardium } \\
(\mathrm{MBq} / \mathrm{mL})\end{array}$ & $\begin{array}{c}\text { Liver } \\
(\mathrm{MBg} / \mathrm{mL})\end{array}$ & $\begin{array}{c}\mathrm{RV} \\
\text { Myocardium } \\
(\mathrm{MBq} / \mathrm{mL})\end{array}$ & $\begin{array}{c}\text { Lung } \\
(\mathrm{MBq} / \mathrm{mL})\end{array}$ & $\begin{array}{c}\mathrm{LV} \mathrm{Blood} \\
(\mathrm{MBq} / \mathrm{mL})\end{array}$ & $\begin{array}{c}\text { Aorta } \\
(\mathrm{MBq} / \mathrm{mL})\end{array}$ & $\begin{array}{c}\text { Right } \\
\text { Atrium } \\
(\mathrm{MBg} / \mathrm{mL})\end{array}$ & $\begin{array}{c}\text { Left } \\
\text { Atrium } \\
(\mathrm{MBg} / \mathrm{mL})\end{array}$ \\
\hline 35.21 & 0.76 & 23.61 & 0.64 & 0.40 & 2.23 & 11.4 & 11.3 \\
$(10.20)$ & $(0.76)$ & $(26.72)$ & $(2.41)$ & $(0.34)$ & $(1.69)$ & $(12.84)$ & $(10.83)$ \\
\hline
\end{tabular}

\subsubsection{Digital Simulations}

The activity distribution for the MOBY simulation was set equal to the organ biodistribution data from Table 4.3. The activity of the organs not displayed in the table was set to equal the activity of blood. The mean SNR from the liver VOIs from Table 4.2 was 7.6, corresponding to a squared SNR of 58 . The plot of the squared SNR versus 
relative activity concentration for the $\left[{ }^{13} \mathrm{~N}\right]$-solution filled syringe is displayed in Figure 4.5. Knowing the true liver squared SNR, the linear relationship $\left(R^{2}>0.99\right)$ was used in combination with the biodistribution data in Table 4.3 to scale the noise added as a function of activity according to equation 4.3. The mean transverse and axial resolutions were used in the simulation.

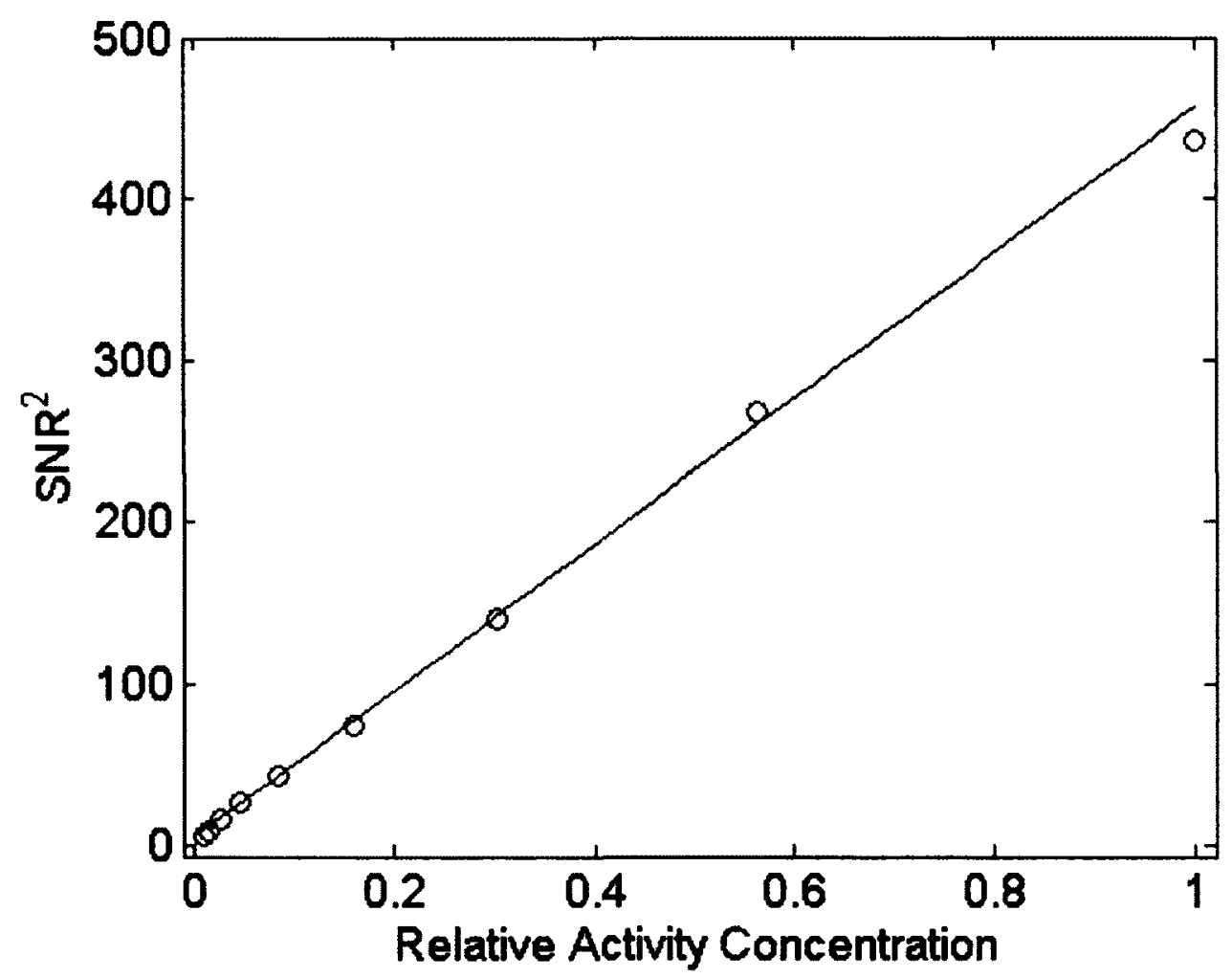

Figure 4.5. Plot of the squared SNR measured in the mice livers as a function of relative activity concentration. The open circles represent the measured data while the straight line is the linear fit $\left(R^{2}>0.99\right)$. 
TABLE 4.4. PV-CORRECTED 3D MOBY SIMULATIONS WITH AND WITHOUT NOISE.

\begin{tabular}{ccccc}
\hline \multirow{2}{*}{ Phantom } & \multicolumn{2}{c}{ Myocardial RC } & \multicolumn{2}{c}{ NU (\%) } \\
\cline { 2 - 5 } & $\begin{array}{c}\text { Before } \\
\text { Correction }\end{array}$ & $\begin{array}{c}\text { After } \\
\text { Correction }\end{array}$ & $\begin{array}{c}\text { Before } \\
\text { Correction }\end{array}$ & $\begin{array}{c}\text { After } \\
\text { Correction }\end{array}$ \\
\hline No Noise & 0.50 & 1.00 & 21.8 & 9.0 \\
Noise \#1 & 0.49 & 1.00 & 22.1 & 10.6 \\
Noise \#2 & 0.50 & 1.01 & 21.4 & 10.8 \\
Noise \#3 & 0.50 & 1.02 & 22.3 & 11.2 \\
Noise \#4 & 0.50 & 1.02 & 21.8 & 10.3 \\
Noise \#5 & 0.50 & 1.01 & 22.1 & 10.8 \\
\hline
\end{tabular}

The results for the PV-corrected simulation data with and without noise are displayed in Table 4.4. Myocardial RCs were obtained by taking the activity estimates and dividing by the true activity. The corrected activities with and without noise were comparable, with an uncorrected recovery of 0.5 and a corrected recovery of approximately unity. Noise did not have a significant effect on the uncorrected recovery values $(p>0.05)$. The average polar map NU from simulations with noise before correction was $21.9 \%$ which was comparable to the $21.8 \%$ NU without noise. The average NU of the PV-corrected data for simulations with noise was $10.7 \%$. Thus, the NU for the noisy data was slightly higher than the $9 \%$ found without noise. Performing additional full LV model iterations changed myocardial activity by less than $0.1 \%$ with the phantom with no noise. With regards to the simulated phantom with noise, performing more than 20 iterations of the full $\mathrm{LV}$ model changed the myocardial RC by less than $0.2 \%$. 


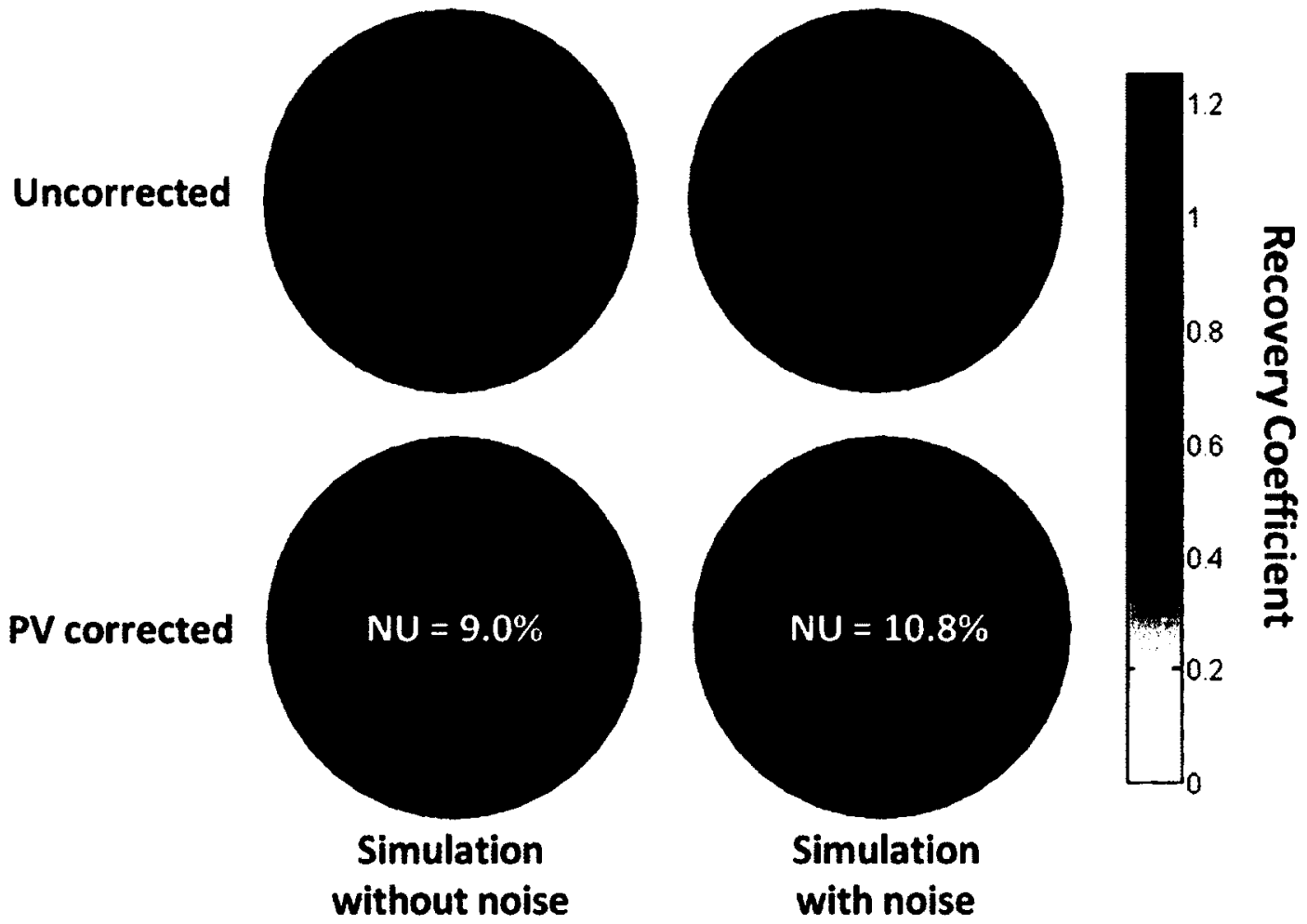

Figure 4.6. Polar maps of the myocardial RCs for the 3D simulated MOBY images with and without noise. The polar map for noise realization 5 from Table 4.4 is displayed.

Figure 4.6 displays the polar maps before and after PV correction for the simulated MOBY phantom data with and without noise. In both cases the polar maps visually confirmed the ability of the algorithm to improve accuracy (RC) and homogeneity (NU) of the activity distribution. The RC for the polar maps from Figure 4.6 was plotted versus LV image plane in Figure 4.7. The plots with and without noise illustrated that the algorithm performed similarly in both cases. The underestimation in the apex was more pronounced on the uncorrected data, but the algorithm was able to correct these data from an $\mathrm{RC}$ of 0.4 to a recovery close to unity. The algorithm 
performed acceptably over all planes, although a slight overestimation of corrected recovery was observed toward the base of the heart.
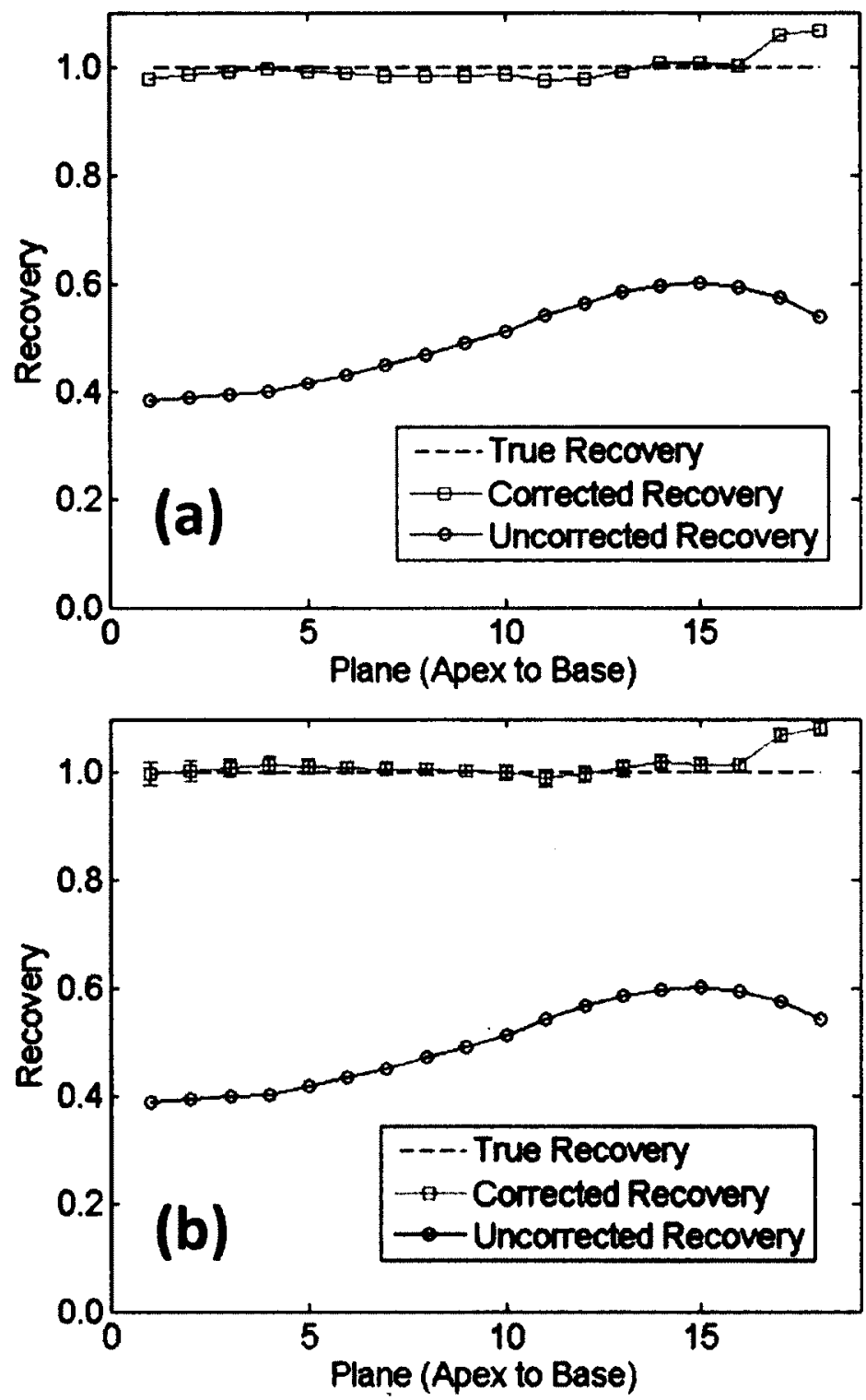

Figure 4.7. Plot of recovery coefficient versus plane, organized from apex to base. The 3D simulated MOBY data (a) without noise and (b) with noise. The error bars of corrected recovery in (b) are computed as the standard deviation across the different noise realizations. Error bars for uncorrected activity fall within the points. 


\subsubsection{Mouse Phantom Simulations and Measurements}

The number of counts from the list-mode data to rebin temporally for the LV mouse phantom and the hollow phantom were based on the LV and blood biodistribution data of Table 4.3. Each sinogram was adjusted to contain approximately the same number of counts as would have been acquired if 35.21 and $0.40 \mathrm{MBq} / \mathrm{mL}$ of activity had been injected into the LV and hollow phantoms respectively and scanned for 225 seconds, which was the length of a single gate in the reconstructed mouse images. The transverse $\times$ axial resolution of the phantom image as determined from the perturbation method was $1.38 \times 2.04 \mathrm{~mm}$. These resolutions were used to perform the PV correction for the measured images and were utilized for generating the simulated phantom data. The mean liver activity, determined from the VOI drawn on the 3D mouse phantom images, was used to transform the activity data from the LV myocardium into RC values. The PVcorrected data for the measured and simulated images are summarized in Table 4.5. The mean calculated wall thickness is also presented.

TABLE 4.5. PV-CORRECTED 3D MOUSE PHANTOM RESULTS FOR SIMULATED AND MEASURED DATA WITH AND WITHOUT SCATTER AND ATTENUATION CORRECTION.

\begin{tabular}{cccc}
\hline Phantom & $\begin{array}{c}\text { Estimated Wall } \\
\text { Thickness } \\
(\mathrm{mm})\end{array}$ & \multicolumn{2}{c}{ Myocardial RC [NU(\%)] } \\
\cline { 3 - 4 } & 0.79 & $\begin{array}{c}\text { Before } \\
\text { Correction }\end{array}$ & After Correction \\
\hline Simulated & $0.48[10.3]$ & $0.97[3.4]$ \\
$\begin{array}{c}\text { Measured - No } \\
\text { Scatter/Attenuation } \\
\text { Correction }\end{array}$ & 0.76 & $0.50[12.5]$ & $1.01[6.5]$ \\
$\begin{array}{c}\text { Measured - With } \\
\text { Scatter/Attenuation } \\
\text { Correction }\end{array}$ & 0.76 & $0.51[10.6]$ & $1.04[5.6]$ \\
\hline
\end{tabular}




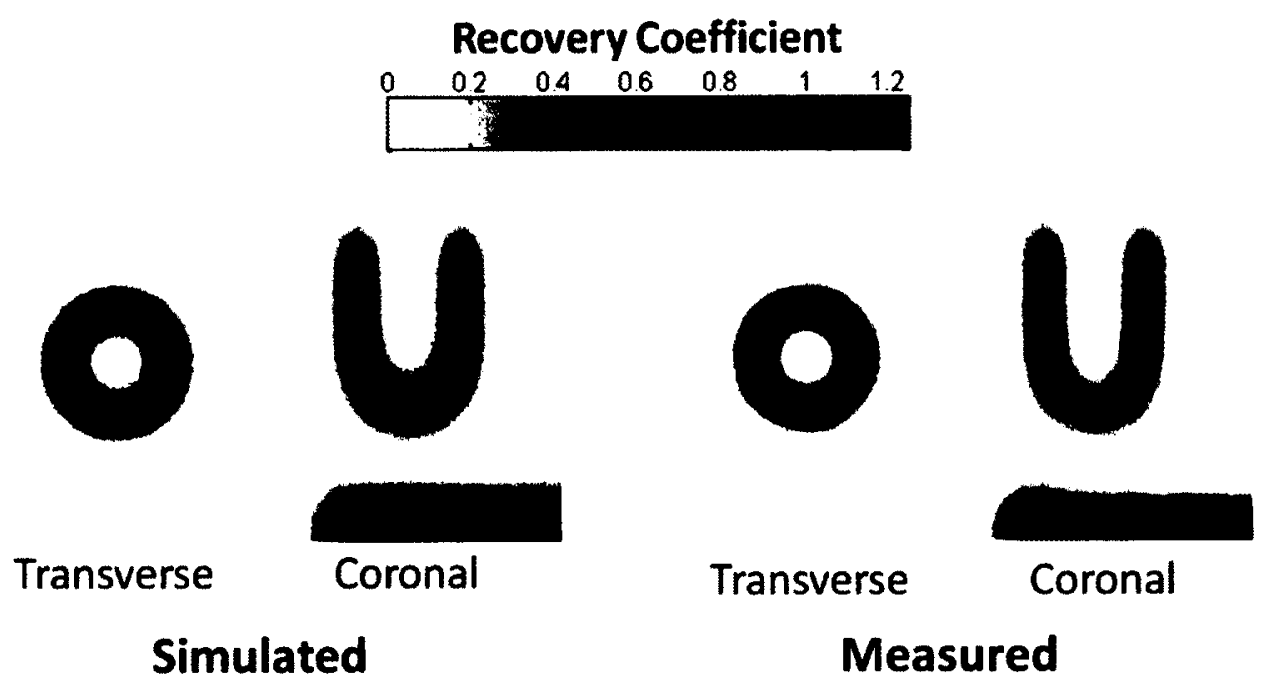

Figure 4.8. The 3D mouse phantom. The transverse and coronal images of the simulated phantom are shown on the left while the transverse and coronal images of the measured phantom with correction for attenuation and scatter are shown on the right.

The PV correction improved the myocardial RC from a 50\% underestimation to a recovery that was within $5 \%$ of the truth. The wall thickness estimated by the PV correction algorithm was underestimated by $10 \%$ in the measured phantoms and by $5 \%$ in the simulated phantom indicating that the errors in wall thickness were larger than the myocardial recovery values. However, the objective of the PV correction was to provide estimates to the myocardial activity and these estimates were found to be more reliable. The NU was decreased in all cases indicating that the PV correction helped restore accuracy and image homogeneity, as anticipated. In terms of accuracy, the measured phantom without corrections for attenuation and scatter had an RC that was closest to the truth although it had the poorest NU before and after correction. There was only a small difference in the PV-corrected image RC values between the measured phantoms with 
and without scatter and attenuation corrections. Furthermore, the NU of the PV-corrected activity values was improved when scatter and attenuation correction were applied. The simulated phantom had the lowest NU before and after correction, and the PV correction of these data had the largest impact on NU, indicating that some of the residual variability from the PV-corrected measured phantom data could have been due to image noise. Images for the measured phantom image, with scatter and attenuation correction, and the simulated image are shown in Figure 4.8. Figure 4.9 presents the polar maps associated with the data of Table 4.5 . Figures 4.8 and 4.9 are displayed on a common scale for comparison.

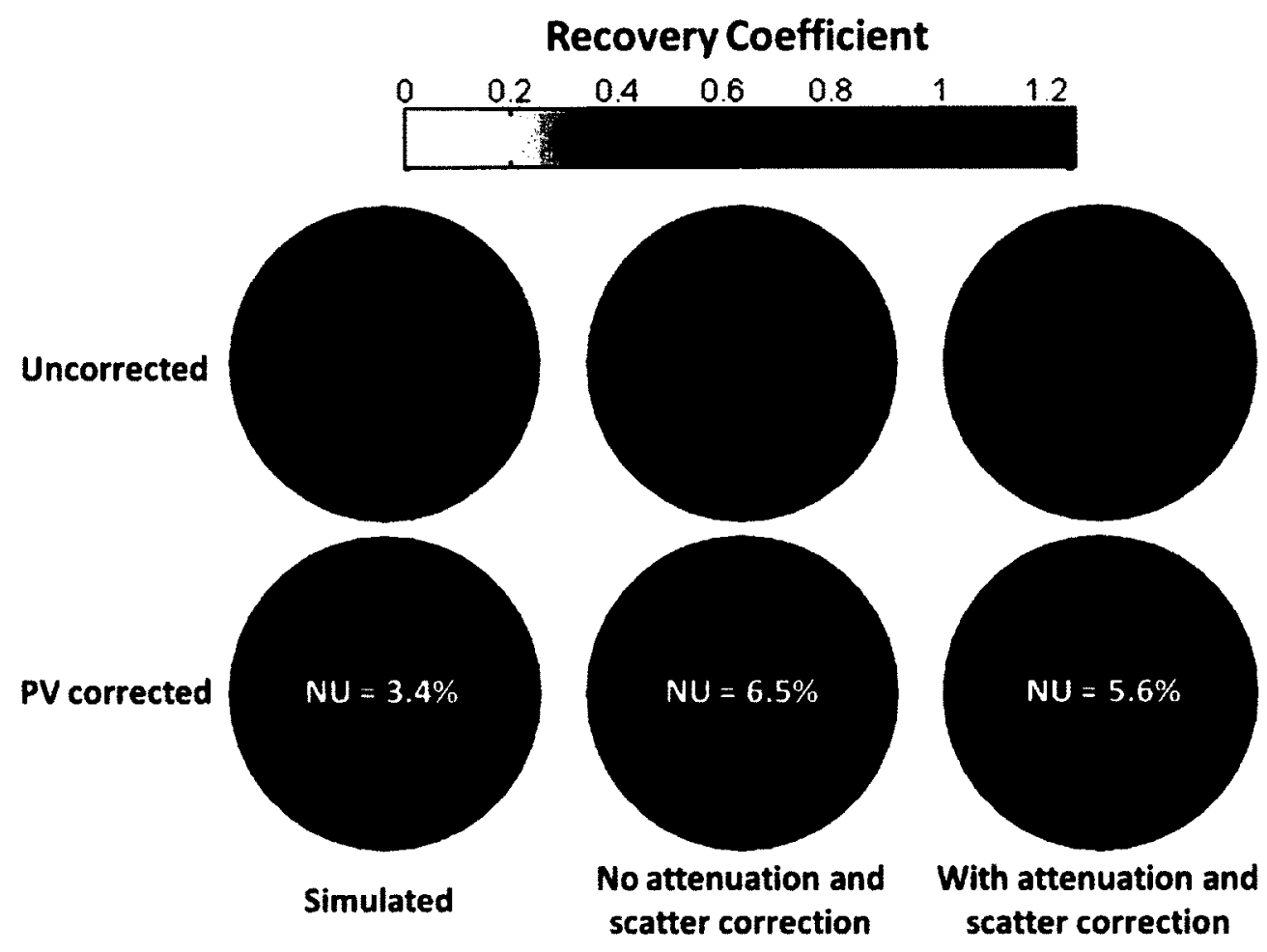

Figure 4.9. Polar maps for the 3D mouse phantom. The top/bottom rows are the polar maps without/with PV correction. The first column is the polar maps for the simulated phantom while the second and third columns are the polar maps for the measured phantom without and with corrections for scatter and attenuation respectively. 


\subsubsection{In-vivo Mouse Imaging}

The uncorrected and PV-corrected mouse data without corrections for scatter and attenuation are presented in Table 4.6 and compared to the measured LV myocardium activity from the biodistribution studies. The PV-corrected values for each mouse were obtained using the resolutions in Table 4.1. The mean myocardial recovery values improved after PV correction from 0.56 to 1.13 ( $p<0.01)$. It was expected that the LV images from healthy mice would have a uniform activity distribution, and thus the NU of the activity distribution should approach zero after correction. The NU was improved on average from $26 \%$ to $17 \%(\mathrm{p}<0.01)$ demonstrating that the algorithm improved quantitative accuracy and aided in restoring homogeneity of the normal mouse LV activity distribution. Similar results were observed with scatter and attenuation correction as shown in Table 4.7. The PV-corrected activities from data without scatter and attenuation correction were significantly different from the biodistribution activities ( $p<0.05,13 \%$ bias). Compared to the results without scatter and attenuation correction, there were no significant differences in PV-corrected recovery $(\mathrm{p}=0.19)$ or $\mathrm{NU}(\mathrm{p}=0.31)$. Therefore, scatter and attenuation correction did not have a significant effect on the PVcorrected activity.

Figure 4.10 shows the LV images of mouse 4 , with attenuation and scatter correction. The figure also displays the polar maps that were generated with FlowQuant, averaged over all gates, before and after PV correction. In this case, the algorithm restored the average recovery to within $2 \%$ of the truth and improved the NU by $10 \%$. The PV-corrected polar maps revealed that the algorithm overestimated the RC slightly 
towards the base and underestimated the RC in the apex region, and the NU of $16 \%$ can be partially attributed to these residual inaccuracies.

TABLE 4.6. PV-CORRECTED MOUSE LV MYOCARDIUM VALUES WITHOUT CORRECTIONS FOR ATTENUATION AND SCATTER.

\begin{tabular}{|c|c|c|c|c|c|c|c|}
\hline \multirow{2}{*}{ Mouse } & \multirow{2}{*}{$\begin{array}{c}\text { Bio-distribution } \\
\text { Activity } \\
\text { (MBq/g) }\end{array}$} & \multirow{2}{*}{$\begin{array}{c}\text { PV- } \\
\text { Corrected } \\
\text { Activity } \\
(\mathrm{MBq} / \mathrm{g})\end{array}$} & \multicolumn{2}{|c|}{$\mathrm{RC}$} & \multirow{2}{*}{$\begin{array}{c}\text { Residual } \\
\text { Bias } \\
(\%)\end{array}$} & \multicolumn{2}{|c|}{ NU (\%) } \\
\hline & & & $\begin{array}{l}\text { Before } \\
\text { Correction }\end{array}$ & $\begin{array}{c}\text { After } \\
\text { Correction }\end{array}$ & & $\begin{array}{l}\text { Before } \\
\text { Correction }\end{array}$ & $\begin{array}{c}\text { After } \\
\text { Correction }\end{array}$ \\
\hline 1 & 25.3 & 25.8 & 0.49 & 1.02 & 2 & 29.1 & 20.7 \\
\hline 2 & 43.0 & 37.4 & 0.46 & 0.87 & -13 & 25.9 & 17.0 \\
\hline 3 & 22.7 & 24.2 & 0.55 & 1.07 & 7 & 27.9 & 22.4 \\
\hline 4 & 39.0 & 38.3 & 0.50 & 0.98 & -2 & 26.7 & 16.0 \\
\hline 5 & 38.9 & 46.1 & 0.57 & 1.19 & 19 & 25.9 & 18.3 \\
\hline 6 & 36.3 & 49.9 & 0.62 & 1.37 & 37 & 27.2 & 13.9 \\
\hline 7 & 31.9 & 39.3 & 0.62 & 1.23 & 23 & 24.7 & 13.6 \\
\hline 8 & 36.0 & 41.3 & 0.58 & 1.15 & 15 & 23.5 & 16.4 \\
\hline 9 & 27.7 & 34.6 & 0.65 & 1.25 & 25 & 24.1 & 14.2 \\
\hline 10 & 40.0 & 48.3 & 0.59 & 1.21 & 21 & 29.3 & 13.9 \\
\hline $\begin{array}{l}\text { Mean } \\
\text { (SD) }\end{array}$ & $\begin{array}{l}34.1 \\
(6.9)\end{array}$ & $\begin{array}{l}38.5^{\dagger} \\
(8.7)\end{array}$ & $\begin{array}{c}0.56 \\
(0.06)\end{array}$ & $\begin{array}{l}1.13^{*} \\
(0.15)\end{array}$ & $\begin{array}{c}13.4 \\
(14.8)\end{array}$ & $\begin{array}{l}26.4 \\
(2.0)\end{array}$ & $\begin{array}{l}16.6^{*} \\
(3.0)\end{array}$ \\
\hline $\begin{array}{l}\text { NU } \\
(\%)\end{array}$ & 20.2 & 22.6 & 10.7 & 13.3 & $111 \%$ & 7.6 & 18.1 \\
\hline
\end{tabular}

* $p<0.01$ compared to values before correction ${ }^{\dagger} p<0.05$ compared to biodistribution values 
TABLE 4.7. PV-CORRECTED MOUSE LV MYOCARDIUM VALUES WITH CORRECTIONS FOR ATTENUATION AND SCATTER.

\begin{tabular}{|c|c|c|c|c|c|c|c|}
\hline \multirow{2}{*}{ Mouse } & \multirow{2}{*}{$\begin{array}{c}\text { Bio-distribution } \\
\text { Activity } \\
(\mathrm{MBq} / \mathrm{g})\end{array}$} & \multirow{2}{*}{$\begin{array}{c}\text { PV- } \\
\text { Corrected } \\
\text { Activity } \\
(\mathrm{MBq} / \mathrm{g})\end{array}$} & \multicolumn{2}{|c|}{$\mathrm{RC}$} & \multirow{2}{*}{$\begin{array}{c}\text { Residual } \\
\text { Bias } \\
(\%)\end{array}$} & \multicolumn{2}{|c|}{ NU (\%) } \\
\hline & & & $\begin{array}{l}\text { Before } \\
\text { Correction }\end{array}$ & $\begin{array}{c}\text { After } \\
\text { Correction }\end{array}$ & & $\begin{array}{l}\text { Before } \\
\text { Correction }\end{array}$ & $\begin{array}{c}\text { After } \\
\text { Correction }\end{array}$ \\
\hline 1 & 25.3 & 25.4 & 0.46 & 1.01 & 1 & 29.7 & 17.3 \\
\hline 2 & 43.0 & 36.8 & 0.43 & 0.86 & -14 & 25.0 & 13.8 \\
\hline 3 & 22.7 & 23.9 & 0.54 & 1.06 & 6 & 27.2 & 21.6 \\
\hline 4 & 39.0 & 38.4 & 0.47 & 0.99 & -1 & 26.7 & 15.5 \\
\hline 5 & 38.9 & 43.6 & 0.54 & 1.12 & 12 & 25.6 & 20.1 \\
\hline 6 & 36.3 & 47.4 & 0.58 & 1.31 & 31 & 26.2 & 13.9 \\
\hline 7 & 31.9 & 38.2 & 0.59 & 1.20 & 20 & 24.2 & 13.3 \\
\hline 8 & 36.0 & 39.1 & 0.55 & 1.09 & 9 & 22.4 & 15.3 \\
\hline 9 & 27.7 & 38.4 & 0.66 & 1.39 & 39 & 23.0 & 12.2 \\
\hline 10 & 40.0 & 45.2 & 0.56 & 1.13 & 13 & 28.7 & 14.2 \\
\hline $\begin{array}{l}\text { Mean } \\
\text { (SD) }\end{array}$ & $\begin{array}{l}34.1 \\
(6.9)\end{array}$ & $\begin{array}{l}37.6^{\dagger} \\
(7.7)\end{array}$ & $\begin{array}{c}0.54 \\
(0.07)\end{array}$ & $\begin{array}{l}1.11^{*} \\
(0.16)\end{array}$ & $\begin{array}{c}11.6 \\
(15.5)\end{array}$ & $\begin{array}{l}25.9 \\
(2.3)\end{array}$ & $\begin{array}{c}15.7^{*} \\
(3.1)\end{array}$ \\
\hline $\begin{array}{l}\mathrm{NU} \\
(\%)\end{array}$ & 20.2 & 20.5 & 13.0 & 14.3 & 134 & 8.9 & 19.7 \\
\hline
\end{tabular}

${ }^{*} p<0.01$ compared to values before correction

$+p=0.06$ compared to biodistribution values 


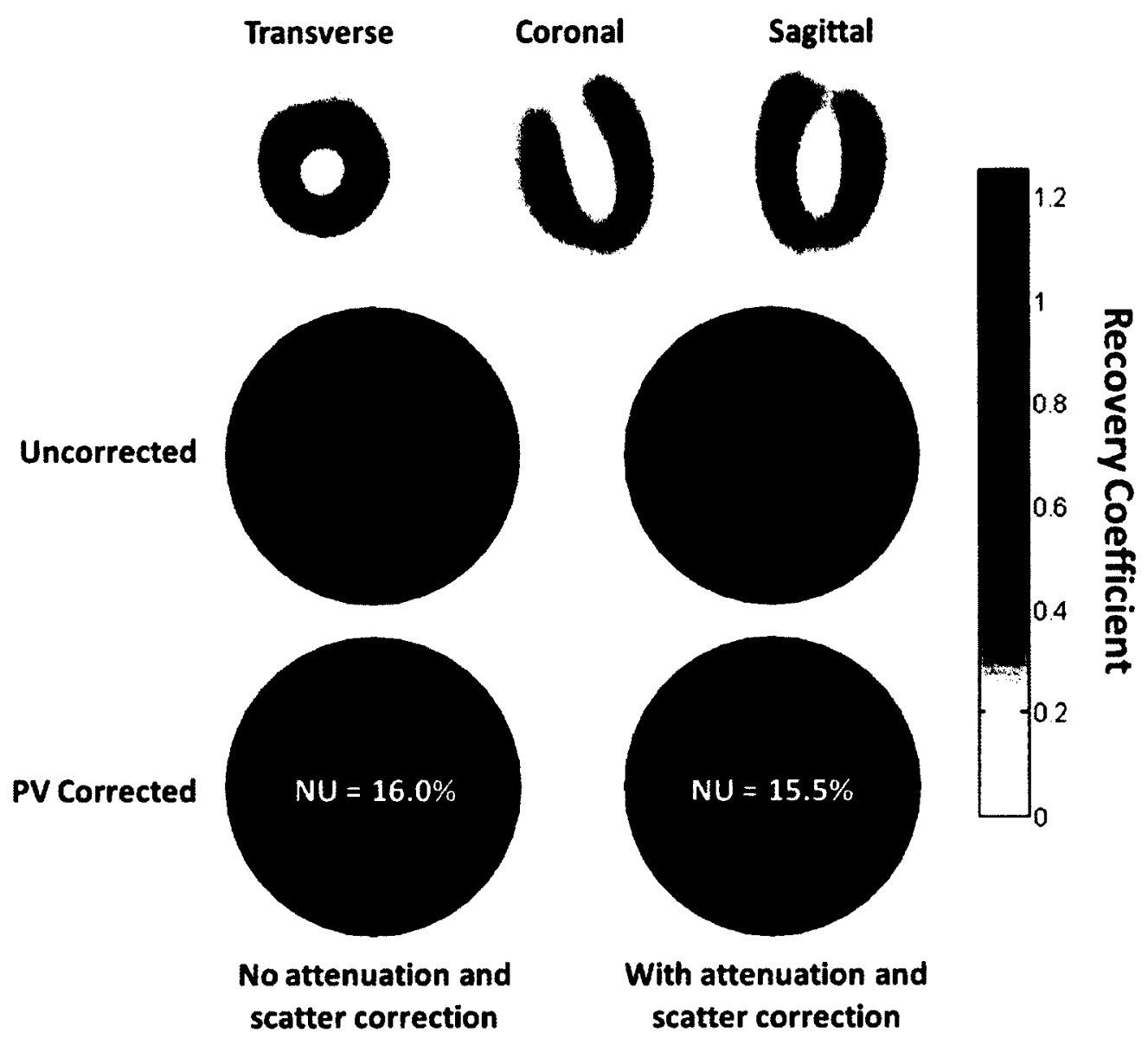

Figure 4.10. Illustration of the PV correction for mouse 4. The LV images at enddiastole with attenuation and scatter correction are shown on the top row. Polar maps, averaged across all gates, before (middle row) and after (bottom row) PV correction are presented for images without (left) and with (right) scatter and attenuation correction. Images are on a common scale for comparison. 


\subsection{Discussion}

The PV correction algorithm improved recovery and uniformity for the mouse images, phantom images and simulated PET images demonstrating the effectiveness of the algorithm to enhance the quantitative accuracy of the LV myocardial activity measurements. While Inveon OSEM3D/MAP reconstruction incorporated resolution modeling (see equation 1.8) that helped improve resolution recovery (Qi et al. 1998b), the uncorrected RCs from Tables 4.5-4.7 suggested that this algorithm was unable to fully maintain quantitation in the mouse heart wall demonstrating the need for additional PV correction. The Inveon scanner and reconstruction algorithm permitted correction for scatter and attenuation for the mouse and 3D phantom data. In the case of mouse imaging, the corrections did not lead to a significant improvement in PV-corrected activity or uniformity. Based on these results, it could be concluded that the effects of scatter and attenuation on quantitative cardiac mouse PET imaging were negligible.

The uniformity results differed between the simulated, phantom and mouse data. The LV mouse phantom data were observed to have a superior uniformity for the measured and simulated data versus the simulated MOBY or measured mouse data. This was due to the LV mouse phantom having only one gate with a constant LV wall thickness. When the PV correction algorithm was executed, the uniformity of the activity distribution inevitably improved. In comparing the uniformity between the MOBY simulations and mouse data, the NU values were slightly higher in the case of the mouse data, although the results before and after PV correction were similar. The inferior uniformity in the mouse data is likely due to inaccurate modeling of the LV shape and slight position-dependence of the PSF. 
In a previous study, a 1D/3D PV correction algorithm was evaluated (Chapter 3). Using a comparable MOBY phantom simulation, the algorithm underestimated the LV myocardial recovery by $10 \%$ on average, and the underestimation was more severe at the apex (Figure 3.5). As was evident from Table 4.4 and Figure 4.7, the 3D algorithm performed well in all planes from apex to base and provided PV-corrected values that were accurate to within $2 \%$. The new algorithm offered improved results by considering a complete 3D geometry of the mouse LV. The improvements were especially noticed at the apex where resolution losses occurred in all directions due to the hemispherical shape in the apex region of the heart.

To evaluate the sensitivity of the algorithm to initial conditions, the initial estimates of wall thickness and myocardial activity were altered to observe how the PVcorrected activity was impacted. Figures 4.11(a) and 4.11(b) demonstrated the percent bias as a function of under/overestimating the initial parameters for wall thickness and myocardial activity respectively for the MOBY simulated data without noise. The studied values were chosen to cover a range of wall thicknesses and activities typical of mouse imaging. Changes in initial parameters of $0 \%$ indicated the default values. Changing the initial wall thickness estimate by $10 \%$ corresponded to a change in the corrected activity of approximately $1 \%$ while a change in the initial activity estimate of $10 \%$ corresponded to a change in the corrected activity of less than $1 \%$. These data suggest that the fitting was robust over a range of initial parameter estimates. 

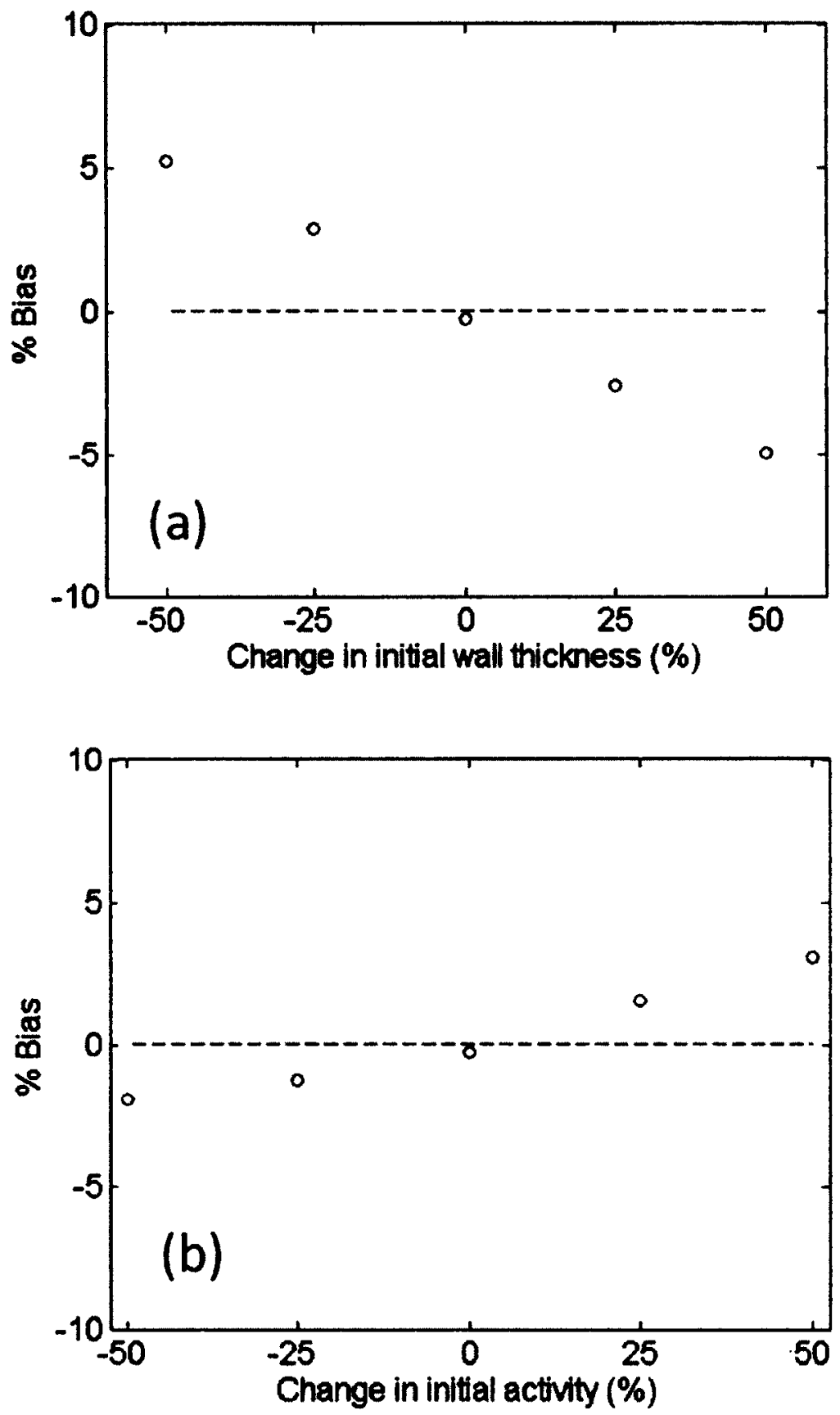

Figure 4.11. Percent bias as a function of change in initial conditions for MOBY simulation. (a) Change in initial wall thickness and (b) change in initial activity. 
The shape of the PSF of the images acquired was assumed Gaussian such that the PV correction algorithm could operate efficiently by exploiting PSF separability. Figure 4.4 demonstrated that a Gaussian function may not describe the actual PSF perfectly. An alternate function to describe PSF shape may have improved the accuracy of the results, and this could be explored in a future study. However, to avoid the computational cost of 3D convolution it would be necessary to maintain PSF separability.

Photon attenuation and scatter were not considered for the ${ }^{22} \mathrm{Na}$ point source measurement. To obtain the PSF, two separate measurements were performed. The attenuation distribution of the point source embedded in acrylic was not perfectly representative of the mouse attenuation distribution. Therefore, the accuracy of the perturbation was limited by photon attenuation. However, the results in this study suggested that scatter and attenuation had negligible effects on mouse heart quantification and it was believed that measuring the PSF in vivo would have minimal effects.

An advantage with the 3D mouse phantom was that the geometry was known and an alternative transverse resolution measurement technique could be performed by convolving a 2D Gaussian with the known activity distribution in the base region (Chapter 3). The FWHM of the 2D Gaussian was adjusted to minimize the sum of squared error between the convolved and measured data and the resolution was found to be $1.33 \mathrm{~mm}$ which was $0.05 \mathrm{~mm}$ less than the perturbation measurement. When this transverse resolution was used, the $\mathrm{RC}$ for the measured phantom with scatter and attenuation correction applied was 0.98 which was more comparable to the recovery of the simulation. The wall thickness estimate was also improved to $0.81 \mathrm{~mm}$ which was closer to the truth and to the simulation (Table 4.5). As discussed in Section 3.2.2 clear 
cast acrylic has a different linear attenuation coefficient in comparison to soft tissue. This could potentially alter the resolution measurements. However, given that scatter and attenuation showed negligible effects for quantification in mice, it was believed that this material would also not significantly alter the resolution measurements. Therefore, these results suggested that fitting a 3D Gaussian to the PSF obtained from the perturbation approach may have overestimated the effective Gaussian resolution slightly, and that to improve accuracy another PSF shape or measuring technique could be explored.

The PV-corrected activity data from the mice overestimated the true activity by $11 \%$ on average (Table 4.7 ) with scatter and attenuation correction applied. The alternative method described here for assessing transverse resolution could not be performed because the actual geometry of the mouse heart was unknown. The sensitivity of the resolution on the PV-corrected activity was tested by lowering the transverse resolution by $0.05 \mathrm{~mm}$ as suggested by the phantom studies. The new resolution was tested on mice 2 and 9, which had the lowest and highest PV-corrected RCs of 0.86 and 1.39 respectively (Table 4.7). The PV-corrected RCs using the lower resolution were decreased to 0.83 and 1.31 , thus the differences in recovery were 3 and $6 \%$. If the PVcorrected data from the mice were lowered by $6 \%$ for all mice, the activity would still be overestimated by $5 \%$ on average. In the case of the 3D phantom, using the lower resolution generated a PV-corrected value that was only $2 \%$ different from the truth.

There are several factors that could contribute to statistical errors. Measurement uncertainties from the gamma-counter counting statistics or from tissue sample weighting propagate into statistical uncertainties on the calculated biodistribution activity. The errors on the counting statistics were less than $0.1 \%$ while errors on weights were less 
than $1 \%$. Manual placement of VOIs over the liver region in the mice could also have introduced errors. Ten independent VOIs were manually drawn on the liver region for one of the mice and the mean liver uptake was found to vary by less than $1 \%$. Biological variability between $L V$ regions also introduces statistical uncertainty to the biodistribution activity. Performing biodistribution with the LV myocardium sectioned into five distinct regions (i.e. apex, and four base regions), showed a $15 \%$ biological variability. Table 4.6 demonstrated that there was a systematic overestimation $(p<0.05)$ when comparing PV-corrected activity to the biodistribution activity. This overestimation suggested that the residual errors were likely to be due to a systematic bias rather than statistical variations. Therefore, the residual errors are more likely to be due to improper resolution estimation or to motion of the mouse during the PET scan. Respiratory motion can introduce blurring into the PET image. In this study, a respiratory gating signal was not acquired. Yang et al. (2005) have shown that there is minimal respiratory motion in mice for more than $75 \%$ of the cycle. Binning list-mode data with additional gates distributes the counts into a larger number of sinograms, thus decreasing SNR in each of the final images. Future studies could focus on eliminating the portion of the cycle that is contaminated by respiratory motion to further increase the accuracy of the PV correction. Translational motion from the mouse settling or physically moving during the scan was also neglected in this study. This motion may have caused additional blurring that was not modeled by the PSF and more work would need to be done to understand this effect. Though it was believed that these motion effects were minor, elimination of all motion artifacts would be expected to improve accuracy. 
The mean Gaussian FWHM value was used to describe the resolution. Variations in the transverse and axial resolutions were $2.1 \%$ and $2.4 \%$ (Section 4.3 .1 ) respectively. The PV correction may have caused local estimations in the activity distribution to be under/overestimated due to the use of a stationary PSF. The use of a position-dependent PSF could increase local accuracy and result in a more uniform activity distribution. However, given that the mean resolution was used and that there were only small variations in resolution it is predicted that improvements in accuracy using a position sensitive PSF would be minimal.

Using anatomical imaging modalities such as $\mathrm{CT}$ or MRI is another viable solution for PV correction. Cine MRI can obtain resolutions on the order of $0.1-0.2 \mathrm{~mm}$ (deKemp et al. 2010) while micro-CT images with respiratory and cardiac gating have been reported to have isotropic voxels on the order of $0.1 \mathrm{~mm}$ (Badea et al. 2008). In comparison to the PV-corrected data for the 3D mouse phantom (Table 4.5), the anatomic imaging modalities have resolutions on the order of the errors in the estimated wall thicknesses. Many anatomic based PV correction techniques, including the GTM approach (Rousset et al. 1998), also rely on a PSF measurement. As discussed, inaccurate PSF modeling can increase uncertainties in the PV-corrected activities. The advantage with anatomic imaging is that the true heart geometry would be known offering additional information that can be used for PV correction. It was expected that PV correction proposed in this work would perform comparably to an anatomical based correction. However, the implemented algorithm had the advantage that no additional gated and segmented anatomic scan was required. 
The overestimation of the base activity of the PV-corrected MOBY simulations (Figure 4.7) indicated that the algorithm could be improved in this region. Beyond the last basal plane, the algorithm assumed that the estimated PV-free image $\left(I_{L V, 3 D}\right)$ could be described by the uncorrected PET image $\left(M_{P E T, 3 D}\right)$. To increase the accuracy of the algorithm, fitting could be performed along the axial direction at the top of the LV myocardium in order to delineate the contours for $I_{L V, 3 D}$. This would help extend activity distribution estimates beyond the base of the heart, and could also aid in improving the accuracy of base activity.

The objective of this study was to PV correct LV myocardial activity in FDG mouse heart imaging. The digital simulations and physical phantom measurements were performed to resemble typical conditions of FDG mouse imaging studies. Mice were studied because they are the most common animal model used to study human disease processes. It is expected that using other tracers can generate similar results provided adequate count statistics and that sufficient contrast exists between myocardium and background for contour detection. Furthermore, the methodology was tested on mice where the spatial resolution is on the order of the myocardial wall thickness. The algorithm could also be used in other species where a similar relationship between spatial resolution and wall thickness is observed. For the purpose of this study, small animal imaging was targeted because of the availability of ex vivo biodistribution which enabled validation of the correction accuracy.

The presented algorithm did not consider variations in activity distribution across the myocardium wall thickness (in the radial direction). Therefore, as in the case of the combined $1 \mathrm{D} / 2 \mathrm{D}$ or $1 \mathrm{D} / 3 \mathrm{D}$ models, the model may underperform in a region with a 
subendocardial infarct. An additional radius and activity parameter could be introduced into the model (Figure 4.1) to account for this. In these cases, anatomic imaging could be valuable to aid in myocardial contour delineation to preserve robust parameter estimations. Although the proposed method has not been evaluated in LV myocardial infarction models, these models are not commonly used in transgenic mouse research studies, evaluating the biologic mechanisms of disease and therapy.

\subsection{Conclusion}

Quantitative accuracy in cardiac mouse PET FDG imaging can be improved using the proposed 3D geometrical PV correction. The presented algorithm improved quantitative accuracy of simulated mouse heart images to within $2 \%$ of the truth on average while the accuracy of the simulated and measured 3D mouse phantom LV myocardial activity was enhanced with PV correction, such that the activity values were within $5 \%$ of the truth. Quantitative accuracy of the LV activity distribution from ECGgated FDG cardiac mouse PET studies was restored to be within $15 \%$ of the true activity using the 3D PV correction. For all phantom and measured data, the PV correction improved the NU by $5-10 \%$. For the cardiac mouse PET studies, scatter and attenuation correction were found to have a negligible effect on PV-corrected LV myocardial activity quantification. 


\section{Chapter 5. Summary and Future Directions}

PV effects occur when the sizes of the structures being imaged are on the order of the image spatial resolution. In cardiac FDG PET imaging, PV effects cause an underestimation of LV myocardial activity which must be corrected to restore quantitative accuracy.

The 1D/2D PV correction algorithm restored LV myocardial activity in the base region to within approximately $5 \%$ for a variety of $2 \mathrm{D}$ simulated and measured PET phantoms. The algorithm performed reliably with different noise levels, background activity, myocardial wall motion and LV wall thickness. The algorithm also improved uniformity of the activity distribution in all cases. Initial results with cardiac FDG mouse PET imaging studies suggested that the algorithm had potential for correcting PV effects in the base region of the LV myocardium.

Cardiac PET imaging is performed in 3D, thus a 2D PV correction algorithm is insufficient for correcting the 3D PV effects. The combined 1D/3D algorithm was created to more accurately consider the 3D structure of the heart. The algorithm improved quantitative accuracy to within approximately $10 \%$ for $3 \mathrm{D}$ simulated data while 
simultaneously improving NU. However, the algorithm was limited in the apex region because the 1D model did not accurately describe the myocardial curvature in this region.

A complete 3D PV correction model was proposed to overcome the limitations anticipated with the combined 1D/3D model. Furthermore, the PSF of the reconstruction algorithm was directly measured using a point source and the perturbation method. The algorithm restored quantitative accuracy to within approximately $2 \%$ for $3 \mathrm{D}$ simulated MOBY PET data with and without noise while improving the spatial NU by $10 \%$. In the case of the static 3D mouse LV phantom, the algorithm improved the myocardial activity to within $5 \%$ while simultaneously improving NU. PV correction restored true activity values to within $10-13 \%$ for ECG-gated FDG cardiac mouse PET studies while improving NU from $26 \%$ to $16 \%$ demonstrating the ability of the 3D PV correction algorithm to perform adequately in vivo. Scatter and attenuation correction were not found to significantly impact cardiac mouse PET image quantification.

The goal of this study was to restore quantitative accuracy due to PV losses for FDG mouse heart imaging. It is predicted that similar results would be obtained if different tracers were used that produced cardiac PET images with a similar activity distribution and SNR in the LV myocardium. $\left[{ }^{13} \mathrm{~N}\right]$-ammonia is a tracer used to evaluate perfusion which can be related to myocardial blood flow (Khorsand et al. 2005) using tracer kinetic modeling. The kinetics of ammonia and FDG are thus different, producing PET images with different activity distributions. The half life of ${ }^{13} \mathrm{~N}$ is also shorter than ${ }^{18} \mathrm{~F}$ (Table 1.1 ), thus for the same injected activity, $\left[{ }^{13} \mathrm{~N}\right]$-ammonia would have lower overall counts than $\left[{ }^{18} \mathrm{~F}\right]-\mathrm{FDG}$ leading to a lower SNR in the myocardium of PET images. Thus, if alternative tracers are considered, each tracer should be validated using the 
methods and phantoms presented in this work prior to being applied to small animal imaging.

Each radioisotope has a unique positron range (Table 1.1). The resolution measurements performed in this study for the $3 \mathrm{D}$ PV correction used a ${ }^{22} \mathrm{Na}$ source which has a similar positron range to ${ }^{18} \mathrm{~F}$ (Chapter 4$)$. When performing PET imaging with other radioisotopes, the width of the PSF is affected due to positron range and the ${ }^{22} \mathrm{Na}$ source may be insufficient in describing the true PSF. Therefore, when measuring the PSF for other isotopes this effect must be considered.

The PSF was assumed Gaussian within this study. In Chapter 4, there was evidence that this model may not be completely accurate. Further studies utilizing the PV correction algorithms proposed in this work could focus on evaluating other PSF shapes, to explore if improvements in accuracy and precision can be obtained.

One of the primary objectives with preclinical PET imaging is to examine mouse models of human disease. Many models of myocardial disease affect heart uptake as a whole. The PV correction technique proposed in this work calculates global uptake, and thus the method should be equally applicable in disease models that affect entire heart uptake such as in gene knock-out or transgenic mouse models of disease (Zaragoza et al. 2011). In the case of surgically induced myocardial disease, such as the ligation of an artery (e.g. induced subendocardial infarct), the PV correction method may not perform as well as discussed in Chapter 4 because it can become difficult to discriminate between myocardium and background. However, surgical disease models are used less frequently and were thus not targeted in this study. 
To validate the accuracy of this algorithm, the PV-corrected activity myocardial values were compared to the true activity distribution in the case of phantom data, and to biodistribution activity data in the case of mouse data. The comparison of the presented PV correction algorithm to other PV correction algorithms was not investigated. A drawback with other state-of-the-art PV correction methods that could be applied to 3D cardiac imaging, such as the GTM method, is that anatomical imaging for clear definition of the LV myocardium and cavity is required. No anatomical imaging was available in this study. However, a future study could be designed to directly compare the GTM method using anatomic imaging with MRI or CT to the proposed methodology to explore benefits from either method.

The effects of PV correction on tracer kinetic modeling could be studied. This study focused on static uptake imaging, but this algorithm could be implemented in 3D dynamic imaging as follows. Gated images are acquired for the late uptake phase and the 3D PV correction is applied to derive segmented LV myocardium images for each ECG gate. Dynamic images are reconstructed separately and the blood, background and myocardial activities are estimated for each dynamic frame by convolving the segmented LV images with the PSF and comparing to the dynamic image in each frame. Applying this pre-processing step prior to performing kinetic modeling could offer improved accuracy for quantification of the physiological processes of interest. 


\section{References}

Alessio A M, Kinahan P E, Champley K M and Caldwell J H 2010a Attenuationemission alignment in cardiac PET/CT based on consistency conditions Med. Phys. 37 1191-2000

Alessio A M, Stearns C W, Tong S, Ross S G, Kohlmyer S, Ganin A and Kinahan P E $2010 \mathrm{~b}$ Application and evaluation of a measured spatially variant system model for PET image reconstruction IEEE Trans. Med. Imaging 29 938-949

Aston J A, Cunningham V J, Asselin M C, Hammers A, Evans A C and Gunn R N 2002 Positron emission tomography partial volume correction: Estimation and algorithms J. Cereb. Blood Flow Metab. 22 1019-1034

Badea C T, Drangova M, Holdsworth D W and Johnson G A 2008 In vivo small-animal imaging using micro-CT and digital subtraction angiography Phys. Med. Biol. 54 S319-S350

Bacharach S L 1992 Positron Emission Tomography of the Heart ed S R Bergmann and B E Sobel (Mount Kisco, New York: Futura Publishing) p 15

Bacharach S L, Libutti S K and Carrasquillo J A 2000 Measuring tumor blood flow with H(2)(15)O: Practical considerations Nucl. Med. Biol. 27 671-676

Baete K, Nuyts J, Van Paesschen W, Suetens P, and Dupont P 2004 Anatomical-based FDG PET reconstruction for the detection of hypo-metabolic regions in epilepsy IEEE Trans. Med. Imaging 23 510-519

Bao Q, Cho S, Li Q, Newport D, Chen M, Leahy R and Chatziioannou A F 2008 Monte carlo based estimation of detector response in a large solid angle preclinical PET imaging system IEEE Nuclear Science Symposium and Medical Imaging Conference Record, (Dresden, Germany, October 19-October 25, 2008) p 5010-5013 
Bao Q, Newport D, Chen M, Stout D B and Chatziioannou 2009 Performance evaluation of the Inveon dedicated PET preclinical tomograph based on the NEMA NU-4 standards J. Nucl. Med. 50 401-408

Bartlett M L, Buvat I, Vaquero J J, Mok D, Dilsizian V and Bacharach S L 1996 Measurement of myocardial wall thickening from PET/SPECT images. Comparison of two methods J. Comput. Assist. Tomogr. 20 473-481

Bergmann S R, Herrero P, Markham J, Weinheimer C J and Walsh M N 1989 Noninvasive quantitation of myocardial blood flow in human subjects with oxygen15-labeled water and positron emission tomography J. Am. Coll. Cardiol. 14 639-652

Berger, M J, Hubbell J H, Seltzer, S M, Chang J, Coursey J S, Sukumar R, Zucker D S, and Olsen K 2010 XCOM: Photon Cross Section Database (version 1.5). [Online] Available: http://physics.nist.gov/xcom [Tuesday, 05-Jul-2011 16:37:02 EDT]. National Institute of Standards and Technology, Gaithersburg, MD, USA.

Boussion N, Hatt M, Lamare F, Bizais Y, Turzo A, Cheze-Le Rest C, Visvikis D 2006 A multiresolution image based approach for correction of partial volume effects in emission tomography Phys. Med. Biol. 51 1857-1876

Boxenbaum H 1980 Interspecies variation in liver weight, hepatic blood flow, and antipyrine intrinsic clearance: extrapolation of data to benzodiazepines and phenytoin $J$ Pharmacokinet Biopharm. 8 165-176

Casey M E, Gadagkar H and Newport D A 1995 A component based method for normalization in volume PET Proc. of the 1995 International Meeting on Fully Three-Dimensional Image Reconstruction in Radiology and Nuclear Medicine (Aixles-Bains, France) p 67-71

Chatziioannou A, Qi J, Moore A, Annala A, Nguyen K, Leahy R and Cherry S R 2000 Comparison of 3-D maximum a posteriori and filtered backprojection algorithms for high-resolution animal imaging with microPET IEEE Trans. Med. Imaging $19507-$ 512

Cherry S R, Shao Y, Silverman RW, Meadors K, Siegel S, Chatziioannou A, Young J W, Jones W, Moyers J C, Newport D, Boutefnouchet A, Farquhar T H, Andreaco M, Paulus M J, Binkley D M, Nutt R and Phelps M E 1997 Micro-PET: a high resolution PET scanner for imaging small animals IEEE Trans. Nucl. Sci. 44 1161-1166

Cherry S R, Sorenson J A and Phelps M E 2003 Physics in Nuclear Medicine Third edition (New York, NY: Saunders)

Cherry S R and Dahlbom M 2004 PET:Molecular Imaging and its Biological Applications ed M E Phelps (New York, NY: Springer-Verlag) p 1-124 
Cho S, Li Q Z, Ahn S, Bai B and Leahy R M 2007 Iterative image reconstruction using inverse Fourier rebinning for fully 3-D PET IEEE Trans. Med. Imaging 26 745-767

Collins K A, Korcarz C E, Shroff S G, Bednarz J E, Fentzke R C, Lin H, Leiden J M and Lang R M 2001 Accuracy of echocardiographic estimates of left ventricular mass in mice Am. J. Physiol. Heart Circ. Physiol. $280 \mathrm{H} 1954-\mathrm{H} 1962$

Constantinescu C C and Mukherjee J 2009 Performance evaluation of an Inveon PET preclinical scanner Phys. Med. Biol. 54 2885-2899

Defrise M, Kinahan P E, Townsend D W, Michel C, Sibomana M, Newport D F 1997 Exact and approximate rebinning algorithms for 3-D PET data IEEE Trans. Med. Imaging 16 145-158.

Defrise M and Kinahan P E 1998 The Theory and Practice of 3D PET ed B Bendriem and D W Townsend(Netherlands: Kluwer Academic Publishers) p 10-53

deKemp R A and Nahmais C 1994 Attenuation correction in PET using single photon transmission measurement Med. Phys. 21 771-7778

deKemp R, Ruddy T, Hewitt T, Dalipaj M and Beanlands R 2000 Detection of serial changes in absolute myocardial perfusion with ${ }^{82} \mathrm{Rb}$ PET J. Nucl. Med. 411426-1435

deKemp R A, Epstein F H, Catana C, Tsui B M W and Ritman E K 2010 Small-animal molecular imaging methods J. Nucl. Med. 51 18S-32S

Dumouchel $\mathrm{T}$ and deKemp R 2008 Count rate performance of the Inveon small animal PET scanner J. Nucl. Med. 49 404P

Dumouchel $\mathrm{T}$ and deKemp R A 2010a Experimental validation of a two dimensional partial volume correction strategy for PET imaging in mice with simulated and measured data Med. Phys. 373902

Dumouchel $\mathrm{T}$ and deKemp R A 2010b Recovery of partial volume losses in cardiac mouse PET imaging using a combined $1 \mathrm{D} / 2 \mathrm{D}$ and a combined $1 \mathrm{D} / 3 \mathrm{D}$ model IEEE Nuclear Science Symposium and Medical Imaging Conference Record (Knoxville, TN, USA, October 30-November 6, 2010) p 2303-2307

Dumouchel $T$ and deKemp R A 2011a A combined 1D/2D partial volume correction strategy for cardiac PET imaging in mice $J$. Nucl. Med. 52 S1:562P

Dumouchel $T$ and deKemp R A $2011 \mathrm{~b}$ Analytical-based partial volume recovery in mouse heart imaging IEEE Trans. Nucl. Sci. 58 110-120

Dumouchel T and deKemp R A 2011c 3D Model-based partial volume correction for cardiac PET imaging in mice J. Nucl. Med. 52 S1:204P 
Fang Y-H D and Muzic R F Jr 2008 Spillover and partial-volume correction for imagederived input functions for small-animal ${ }^{18}$ F-FDG PET studies $J$. Nucl. Med. $49606-$ 614

Feng D, Li X and Huang S-C 1996 A new double modeling approach for dynamic cardiac PET studies using noise and spillover contaminated LV measurements IEEE Trans. Biomed. Eng. 43 319-327

Fessler J A and Rogers L 1996 Spatial resolution properties of penalized-likelihood image reconstruction: space invariant tomgraphs IEEE Trans. Imag. Processing 9 $1346-1358$

Freiberg J, Hove J D, Kofoed K F, Fritz-Hansen T, Holmb S, Larsson H B and Kelbæk H 2004 Absolute quantitation of the left ventricular wall and cavity parameters using ECG-gated PET J. Nucl. Card. 11 38-46

Gao X M, Dart A M, Dewar E, Jennings G and Du X J 2000 Serial echocardiographic assessment of left ventricular dimensions and function after myocardial infarction in mice, Cardiovasc. Res. 45 330-338

Gambhir S S 1990 Quantitation of the physical factors affecting the tracer kinetic modeling of cardiac positron emission tomography data $P h D$ dissertation University of California, Los Angeles, CA, United States of America

Gardin J M, Siri F M, Kitsis R M, Edwards J G and Leinwand L A 1995 Echocardiographic assessment of left ventricular mass and systolic function in mice Circ. Res. 76 907-914

Green L A, Gambhir S S, Srinivasan A, Banerjee P K, Hoh C K, Cherry S R, Sharfstein S, Barrio J R, Herschman H R and Phelps ME 1998 Noninvasive methods for quantitating blood time-activity curves from mouse PET images obtained with fluorine-18-fluorodeoxyglucose J. Nucl. Med. 39 729-734

Green L A, Nguyen K, Berenji B, lyer M, Bauer E, Barrio J R, Namavari M, Satyamurthy N and Gambhir S S 2004 A tracer kinetic model for ${ }^{18} \mathrm{~F}-\mathrm{FHBG}$ for quantitating herpes simplex virus type 1 thymidine kinase reporter gene expression in living animals using PET $J$. Nucl. Med. 45 1560-1570

Hoffman E J, Huang S C and Phelps M E 1979 Quantitation in positron emission computed tomography: 1. Effect of object size J. Comput. Assist. Tomogr. 3 299-308

Hoffmann M, Pichler B, Scholkopf B and Beyer T 2009 Toward quantitative PET/MRI: a review of MR-based attenuation correction techniques Eur. J. Nucl. Med. Mol. Imaging 36 S93-S104 
Huang S C, Schwaiger M, Carson R E, Carson J, Hansen H, Selin C, Hoffman E J, MacDonald N, Schelbert H R and Phelps M E 1985 Quantitative measurement of myocardial blood flow with oxygen-15 water and positron computed tomography: an assessment of potential and problems $J$. Nucl. Med. 26 616-625

Huesman R H 1984 A new fast algorithm for the evaluation of regions of interest and statistical uncertainty in computed tomography Phys. Med. Biol. 29 543-552

Hudson H M and Larkin R S 2994 Accelerated image reconstruction using ordered subsets of projection data IEEE Trans Med Imag. 13 601-609

Hutchins G D, Caraher J M and Raylman R R 1992 A region of interest strategy for minimizing resolution distortions in quantitative myocardial PET studies $J$. Nucl. Med. 33 1243-1250

Hutchins G D, Miller M A, Soon V C and Receveur T 2008 Small animal PET imaging Institute for Laboratory Animal Research J. 49 54-65

Iida H, Kanno I, Takahashi A, Miura S, Murakami M, Takahashi, K, Ono Y, Shishido F, Inugami A, Tomura $\mathrm{N}$ et al 1988 Measurement of absolute myocardial blood flow with $\mathrm{H}_{2}{ }^{15} \mathrm{O}$ and dynamic positron-emission tomography. Strategy for quantification in relation to the partial-volume effect Circ. 78 104-115

lida H, Rhodes C G, de Silva R, Yamamoto Y, Araujo L I, Maseri A and Jones T 1991 Myocardial tissue fraction - Correction for partial volume effects and measure of tissue viability $J$. Nucl. Med. 32 2169-2175

Johnson N, Sdringola S and Gould K L 2011 Partial volume correction incorporating Rb82 positron range for quantitative myocardial perfusion PET based on systolicdiastolic activity ratios and phantom measurements $J$. Nucl. Card. 18 247-258

Karp J S, Muehllehnew G, Qu H and Yan X-H 1995 Singles Transmission in volumeimaging PET with a ${ }^{137}$ Cs source Phys. Med. Bio. 40 929-944

Kemp B J, Hruska C B, McFarland A R, Lenox M W and Lowe V J 2009 NEMA NU 22007 performance measurements of the Siemens Inveon ${ }^{\mathrm{TM}}$ preclinical small animal PET system Phys. Med. Biol. 54 2359-2376

Khorsand A, Graf S, Frank H, Kletter K, Sochor H, Maurer G, Schuster E, Globits S, Dudczak R and Porenta G 2003 Model-based analysis of electrocardiography-gated cardiac F-18-FDG PET images to assess left ventricular geometry and contractile function $J$. Nucl. Med. 44 1741-1746

Khorsand A, Graf S, Pirich C, Muzik, O, Kletter K, Dudczak R, Maurer G, Sochor H, Schuster E and Porenta G 2005 Assessment of myocardial perfusion by dynamic N- 
13 ammonia PET imaging: comparison of 2 tracer kinetic models J. Nucl. Cardiol. 12 $410-417$

Kirov A S, Piao J Z and Schmidtlein C R 2008 Partial volume effect correction in PET using regularized iterative deconvolution with variance control based on local topology Phys. Med. Biol. 53 2577-2591

Klein R, Renaud J M, Ziadi, M C, Thorn S L, Adler, A, Beanlands, R S and deKemp R A 2010 Intra- and inter-operator repeatability of myocardial blood flow and myocardial flow reserve measurements using Rubdium-82 PET and a highly automated analysis program J. Nucl. Cardiol.17 600-616

Lange $\mathrm{K}$ and Carson $\mathrm{R} 1984 \mathrm{EM}$ reconstruction algorithms for emission and transmission tomography J Comput Assist Tomogr. 8 306-316

Leahy R M and Qi J 2000 Statistical approaches in quantitative positron emission tomography Stat. Comput. 10 147-165

Lecomte R 2004 Technology challenges in small animal PET imaging Nucl. Instr. Meth. Phys. Res. A 527 157-165

Levin C S and Zaidi H 2007 Current trends in preclinical PET system design PET Clin. 2 $125-160$

Lodge M A, Carson R E, Carrasquillo J A, Whatley M, Libutti S K and Bacharach S L 2000 Parametric images of blood flow in oncology PET studies using $\left[{ }^{15} \mathrm{O}\right]$ water $J$. Nucl. Med. 41 1784-1792

Lortie M, Beanlands R S B, Yoshinaga K, Klein R, DaSilva J N and deKemp R A 2007 Quantification of myocardial blood flow with ${ }^{82} \mathrm{Rb}$ dynamic PET imaging Eur. $J$. Nucl. Med. Mol. Imag. 34 1765-1774

Maroy R, Boisgard R, Comtat C, Frouin V, Cathier P, Duchesnay E, Dolle F, Nielsen P $E$, Trebossen $R$ and Tavitian B 2008 Segmentation of rodent whole-body dynamic PET images: An unsupervised method based on voxel dynamics IEEE Trans. Med. Imaging 27 342-354

Maroy R, Boisgard R, Comtat C, Jego B, Fontyn Y, Jan S, Dubois A, Trebossen R and Tavitian B 2010 Quantitative organ time activity curve extraction from rodent PET images without anatomical prior Med. Phys. 37 1507-1517

Meltzer C C, Zubieta J K, Links J M, Brakeman P, Stumpf M J and Frost J J 1996 MRbased correction of brain PET measurements for heterogeneous gray matter radioactivity distribution $J$. Cereb. Blood Flow Metab. 16 650-658 
Mintzer R A and Siegel S B 2007 Design and performance of a new pixilated LSO/PSPMT gammy-ray detector for high resolution PET imaging IEEE Nuclear Science Symposium and Medical Imaging Conference Record, (Honolulu, HI, USA, October 28-November 3, 2007) p 3418-3422

Moore S C and El Fakhri G 2007 Cardiac PET and PET/CT Imaging ed F Di Carli and M J Lipton (New York, NY: Springer) p 46- 72

Moses W W and Derenzo S E Empirical observation of resolution degradation in positron emission tomographs utilizing block detectors $J$. Nucl. Med. $34101 \mathrm{P}$

Müller-Gärtner H W, Links J M, Prince J L, Bryan R N, McVeigh E, Leal J P, Davatzikos C and Frost J J 1992 Measurement of radiotracer concentration in brain gray matter using positron emission tomography: MRI-based correction for partial volume effects J. Cereb. Blood Flow Metab. 12 571-583

Muzic R F, Chen C H and Nelson A D 1998 A method to correct for scatter, spillover, and partial volume effects in region of interest analysis in PET IEEE Trans. Med. Imaging 17 202-213

National Electrical Manufacturers Association (NEMA) 2008 Performance measurements for small animal positron emission tomographs NEMA Standards Publication NU 4 - 2008 (Rosslyn, VA: NEMA)

Nekolla S G, Martinez-Moeller A and Saraste A 2009 PET and MRI in cardiac imaging from validation studies to integrated applications Eur. J. Nucl. Med. Mol. Img. 36 $121-130$

Nuyts J, Mortelmans L, Suetens P, Oosterlinck A and de Rou M 1989 Model-based quantification of myocardial perfusion images from SPECT $J$. Nucl. Med. $301192-$ 2001

Nuyts J, Maes A, Vrolix M, Schiepers C, Schelbert H, Kuhle W, Bormans G, Poppe G, Buxton D, Suetens P, et al 1996 Three-dimensional correction for spillover and recovery of myocardial PET images $J$. Nucl. Med. 37 767-774

Panin V Y, Kehren F, Michel C and Casey M 2006 Fully 3-D PET reconstruction with system matrix derived from point source measurements IEEE Trans. Med. Imaging 25 907-921

Pollick C, Hale S L and Kloner R A 1995 Echocardiographic and cardiac Doppler assessment in mice J. Am. Soc. Echocardiogr. 8 602-610

Porenta G, Kuhle W, Sinha W, Krivokapich J, Czernin J, Gambhir S S, Phelps M E and Schelbert H R 1995 Parameter estimation of cardiac geometry by ECG-gated PET 
imaging: Validation using magnetic resonance imaging and echocardiography $J$. Nucl. Med. 36 1123-1129

Pysz M A, Gambhir S S and Willmann J K 2010 Molecular imaging: current status and emerging strategies Clin. Radiol. 65 500-516

Qi J, Leahy R M, Hsu C, Farquhar T H and Cherry S R 1998a Fully 3D Bayesian image reconstruction for the ECAT EXACT HR+ IEEE Trans. Nucl. Sci. 45 1096-1103

Qi J, Leahy R, Cherry S R, Chatziioannou A and Farquhar T H 1998b High resolution 3D Bayesian image reconstruction using the microPET small-animal PET scanner Phys. Med. Biol. 43 1001-1013

Qi J and Leahy R M 2000 Resolution and noise properties of MAP reconstruction for fully 3D PET IEEE Trans. Med. Imaging 19 493-506

Qi J and Leahy R M 2006 Iterative reconstruction techniques in emission computed tomography Phys. Med. Biol. 51 R541-R578

Rottman J N, Ni G and Brown M 2007 Echocardiographic evaluation of ventricular function in mice Echocardiography 24 83-89

Rousset O G, Ma Y and Evans A C 1998 Correction for partial volume effects in PET: Principle and validation J. Nucl. Med. 39 904-911

Rousset O, Rahmim A, Alavi A, and Zaidi H 2007 Partial volume correction strategies in PET PET Clin. 2 235-249

Segars W P, Tsui B M W, Frey E C, Johnson A and Berr S S 2004 Development of a 4-D digital mouse phantom for molecular imaging research Mol. Imag. Biol. 6 149-159

Shepp L A and Vardi Y 1982 Maximum likelihood reconstruction for emission tomography IEEE Trans Med Imag. 1 113-122.

Sinusas A J, Bengel F, Nahrendorf M, Epstein F H, Wu J C, Villanueva F S, Fayad Z A and Gropler R J 2008 Multimodality cardiovascular molecular imaging, part I Circ. Cardiovasc. Imaging $1244-256$

Snyder W S, Cook M J, Nasset E S, Karhausen L R, Howells G P and Tipton I H 1975 Report of the task group on reference man ICRP Publication 23 (Pergamon, Oxford)

Soret M, Bacharach S L and Buvat I 2007 Partial-volume effect in PET tumor imaging $J$. Nucl. Med. 48 932-945 
Stegger L, Heijman E, Schäfers K P, Nicolay K, Schäfers M A and Strijkers G J 2009 Quantification of left ventricular volumes and ejection fraction in mice using PET, compared with MRI $J$. Nucl. Med. 50 132-138

Strother S C, Casey M E and Hoffman E J 1990 Measuring PET scanner sensitivity: Relating count rates to image signal-to-noise ratio using noise equivalent counts IEEE Trans. Nucl. Sci. 37 783-788

Sureau F C, Reader A J, Comtat C, Leroy C, Ribeiro M-J, Buvat I and Trebossen R 2008 Impact of image-space resolution modeling for studies with the high-resolution research tomograph J. Nucl. Med. 49 1000-1008

Taegtmeyer H 1984 Six blind men explore an elephant: Aspects of fuel metabolism and the control of tricarboxylic acid cycle activity in the heart muscle Basic Res. Cardiol. $79322-326$

Taegtmeyer H 1996 Cardiac Positron Emission Tomography ed M Schwaiger (Norwell, MA: Kluwer Academic Publishers) p 79 - 95

Teo B K, Seo Y, Bacharach S L, Carrasquillo J A, Libutti S K, Shukla H, Hasegawa B H, Hawkins R A and Franc B L 2007 Partial-volume correction in PET: validation of an iterative postreconstruction method with phantom and patient data $J$. Nucl. Med. 48 802-810

Tohka J and Reilhac A 2008 Deconvolution-based partial volume correction in Raclopride-PET and Monte Carlo comparison to MR-based method Neuroimage 39, 1570-1584

Visser E P, Disselhorst J A, Brom M, Laverman P, Gotthardt M, Oyen W J G and Boerman O C 2009 Spatial resolution and sensitivity of the Inveon small-animal PET scanner J. Nucl. Med. 50 139-147

Wassenaar R W and deKemp R A 2006 Characterization of PET partial volume correction for variable myocardial wall thicknesses IEEE Trans. Nucl. Sci. 53 175180

Watson C C 2000 New, faster, image-based scatter correction for 3D PET IEEE Trans. Nucl. Sci. 47 1587-1594

Watson C C, Townsend D W and Bendriem B 2004 Emission Tomography ed M N Wernick and J N Aarsvold (San Diego, CA: Elsevier) p 195-212

Yang Y, Rendig S, Siegel S, Newport D F and Cherry S R 2005 Cardiac PET imaging in mice with simultaneous cardiac and respiratory gating Phys. Med. Biol. 50 29792989 
Youn H J, Rokosh G, Lester S J, Simpson P, Schiller N B and Foster E: 1999 Twodimensional echocardiography with a $15-\mathrm{MHz}$ transducer is a promising alternative for in vivo measurement of left ventricular mass in mice. J. Am. Soc. Echocardiogr. $1270-75$

Yu S K and Nahmais C 1995 Single-photon transmission measurement in positron tomography using ${ }^{137}$ Cs Phys. Med. Biol. 40 1255-1266

Zaidi $\mathrm{H}$ and Montandon M L 2007 Scatter compensation techniques in PET PET Clin. 2 219-234

Zaragoza C, Gomez-Guerrero C, Martin-Ventura J L, Bianco-Colio L, Begoña L, Mallavia B, Tarin C, Mas S, Ortiz A and Egido J 2011 Animal models of cardiovascular disease J. Biomed. Biotechnol. 2011:497841 